\title{
Expansion schemes for gravitational clustering: computing two-point and three-point functions
}

\begin{abstract}
P. Valageas
Institut de Physique Théorique, CEA Saclay, 91191 Gif-sur-Yvette, France

e-mail: valag@spht.saclay.cea.fr

Received 15 November 2007 / Accepted 19 March 2008

ABSTRACT

Aims. We describe various expansion schemes that can be used to study gravitational clustering. Obtained from the equations of motion or their path-integral formulation, they provide several perturbative expansions that are organized in a different fashion or involve different partial resummations. We focus on the two-point and three-point correlation functions, but these methods also apply to all higher-order correlation and response functions.

Methods. We present the general formalism, which holds for the gravitational dynamics and for any similar models, such as the Zeldovich dynamics, that obey similar hydrodynamical equations of motion with a quadratic nonlinearity. We give our explicit analytical results up to one-loop order for the simpler Zeldovich dynamics. For the gravitational dynamics, we compare our one-loop numerical results with numerical simulations.

Results. We check that the standard perturbation theory is recovered from the path integral by expanding over Feynman's diagrams. However, the latter expansion is organized in a different fashion, and it contains some UV divergences that cancel out as we sum all diagrams of a given order. Resummation schemes modify the scaling of tree and one-loop diagrams, which exhibit the same scaling over the linear power spectrum (contrary to the standard expansion). However, they do not significantly improve over standard perturbation theory for the bispectrum, unless one uses accurate two-point functions (e.g. a fit to the nonlinear power spectrum from simulations). Extending the range of validity to smaller scales, to reach the range described by phenomenological models, seems to require at least two-loop diagrams.
\end{abstract}

Key words. gravitation - cosmology: theory - cosmology: large-scale structure of Universe

\section{Introduction}

According to standard cosmological scenarios, the large-scale structures of the present Universe arise from the amplification by gravitational instability of small primordial fluctuations (Peebles 1980). Then, from observations of the recent Universe at redshifts $z<5$, through galaxy surveys (Tegmark et al. 2006; Cole et al. 2005), weak-lensing studies (Massey et al. 2007; Munshi et al. 2007), or studies of baryonic oscillations (Eisenstein et al. 1998, 2005), we can derive constraints on the cosmological parameters and on the properties of those primordial fluctuations. This requires accurate theoretical predictions for the evolution of gravitational clustering to compare cosmological models with observations. On large scales, where density fluctuations are small, linear theory is sufficient. On small scales, deep into the nonlinear regime associated with virialized structures such as galaxy clusters, one can use numerical simulations or phenomenological models, such as the halo model (Cooray \& Sheth 2002), which allow for an accuracy near $10 \%$. However, many observational probes (such as weak lensing or baryonic oscillations) are mostly sensitive to intermediate scales (wavenumbers $k \sim 0.1 h \mathrm{Mpc}^{-1}$ at $z \lesssim 1$ ) that might be studied through analytical methods, such as perturbative expansions.

Thus, there has recently been a renewed interest in building analytical methods that could improve over the standard perturbation theory and provide reliable predictions in this weakly nonlinear regime. Crocce \& Scoccimarro (2006a,b) present a partial resummation of the diagrammatic series associated with the response function, which recovers the expected decay into the nonlinear regime, in agreement with numerical simulations. This allows one to obtain a more accurate prediction for the matter power spectrum than with the usual perturbation theory (Crocce \& Scoccimarro 2007). On the other hand, Valageas (2004, 2007a) described the path-integral formalism associated with the cosmological gravitational dynamics. This allows one to apply the usual tools of field theory, such as large- $N$ and semiclassical expansions. This provides several methods to perform partial resummations of the usual perturbation theory. Next, Matarrese \& Pietroni (2007a,b) proposed to use the dependence of the system on a high- $k$ cutoff to derive some resummation schemes through the path-integral formalism. An alternative approach developed by McDonald (2007), based on a renormalization group technique, also permits going beyond the standard perturbative expansion.

These works have focused on two-point functions, such as the matter power spectrum or two-point correlation, since the latter are the main quantities obtained from observations. However, the additional information included in higher-order statistics, such as the three-point correlation, can prove very useful in breaking degeneracies (Sefusatti et al. 2006; Munshi et al. 2007). Therefore, we describe in this paper how such methods can be used to compute higher-order functions. We focus on the bispectrum, which is the quantity of most practical interest, since higher-order statistics obtained from observational surveys are increasingly noisy. We consider both the exact gravitational 
dynamics and the simpler Zeldovich dynamics. Indeed, the equations of motion associated with both systems are similar and these expansion schemes apply in the same manner to both (Valageas 2007b). Then, the simpler Zeldovich dynamics can be used as a convenient benchmark to track possible errors in the derivation of the equations associated with various schemes.

This article is organized as follows. First, in Sect. 2 we recall the equations of motion associated with cosmological gravitational clustering. We focus on weakly nonlinear scales hence we restrict ourselves to the hydrodynamical description. We write the path-integral formulation of this system and we briefly present two large- $N$ methods which can be derived within this formalism. We also recall the main properties of the simpler Zeldovich dynamics. Next, we present in a general manner various perturbative expansions that can be applied to both cosmological dynamics (or any equation of motion of the same form, i.e. with a quadratic nonlinearity). Thus, we first recall the standard perturbative expansion over powers of the linear density field in Sect. 3, we describe the equivalent expansion that can be derived from the path-integral formalism by expanding over the interaction term of the action in Sect. 4, we present the resummations associated with the large- $N$ methods in Sect. 5, and we briefly discuss in Sect. 6 the resummation associated with the random advection of small-scale structures by the larger-scale coherent flow (involving a high- $k$ limit). Then, we apply these methods to the Zeldovich dynamics in Sect. 7, up to one-loop order. Indeed, the expressions obtained in this case are simpler than for the gravitational dynamics. This allows us to show explicitly the connection between the different methods and to point out the possible divergences which may appear in intermediate steps as diagrams are gathered in different manners. Finally, we apply these methods to the actual gravitational dynamics in Sect. 8, again up to one-loop order, and we compare their predictions with numerical simulations. We conclude in Sect. 9.

The reader who is not directly interested in the theoretical aspects of gravitational clustering (e.g. the discussion of the various formalisms and perturbative methods that may be built, and the problems, such as UV divergences, that one may encounter) might first read Sect. 8.4 and Figs. 20, 21. There, we compare the standard perturbation theory with the most efficient resummation scheme considered in this paper (at one-loop order), as well as a recent phenomenological model and numerical simulations.

\section{Equations of motion}

\subsection{Fluid dynamics}

In this section, we recall the equations that describe the dynamics of gravitational clustering in a cosmological framework. As in Valageas (2007a), we focus on weakly nonlinear scales where we can use the single-stream approximation. Then, both cold dark matter and baryonic matter are described as a pressureless fluid governed by the continuity and Euler equations, supplemented by the Poisson equation for the gravitational potential $\phi$ (Peebles 1980),

$$
\begin{aligned}
& \frac{\partial \delta}{\partial \tau}+\nabla \cdot[(1+\delta) \boldsymbol{v}]=0, \\
& \frac{\partial \boldsymbol{v}}{\partial \tau}+\mathcal{H} \boldsymbol{v}+(\boldsymbol{v} \cdot \nabla) \boldsymbol{v}=-\nabla \phi, \\
& \Delta \phi=\frac{3}{2} \Omega_{\mathrm{m}} \mathcal{H}^{2} \delta,
\end{aligned}
$$

where $\tau=\int \mathrm{d} t / a$ is the conformal time (and $a$ the scale factor), $\mathcal{H}=\mathrm{d} \ln a / \mathrm{d} \tau$ the conformal expansion rate, and $\Omega_{\mathrm{m}}$ the matter density cosmological parameter. Here, $\delta$ is the matter density contrast and $v$ the peculiar velocity. Since the vorticity field decays within linear theory (Peebles 1980), we take the velocity to be a potential field so that $v$ is fully specified by its divergence $\theta=\nabla . v$. It is convenient to work in Fourier space, which we normalize as

$\delta(\boldsymbol{k})=\int \frac{\mathrm{d} \boldsymbol{x}}{(2 \pi)^{3}} \mathrm{e}^{-\mathrm{i} \boldsymbol{k} \cdot \boldsymbol{x}} \delta(\boldsymbol{x})$,

and to introduce the time coordinate $\eta$ defined from the linear growing mode $D_{+}$as

$\eta=\ln D_{+}(\tau) \quad$ with $\quad D_{+}(z=0)=1$.

Then, as in Crocce \& Scoccimarro (2006a), we define the twocomponent vector $\psi$ as

$\psi(\boldsymbol{k}, \eta)=\left(\begin{array}{l}\psi_{1}(\boldsymbol{k}, \eta) \\ \psi_{2}(\boldsymbol{k}, \eta)\end{array}\right)=\left(\begin{array}{c}\delta(\boldsymbol{k}, \eta) \\ -\theta(\boldsymbol{k}, \eta) / \mathcal{H} f\end{array}\right)$

where $f(\tau)$ is defined as

$f(\tau)=\frac{\mathrm{d} \ln D_{+}}{\mathrm{d} \ln a}=\frac{1}{\mathcal{H}} \frac{\mathrm{d} \ln D_{+}}{\mathrm{d} \tau}$

Then, the equations of motion (1)-(3) read (Crocce \& Scoccimarro 2006a; Valageas 2007a):

$O\left(x, x^{\prime}\right) . \psi\left(x^{\prime}\right)=K_{\mathrm{s}}\left(x ; x_{1}, x_{2}\right) . \psi\left(x_{1}\right) \psi\left(x_{2}\right)$,

where we have introduced the coordinate $x=(\boldsymbol{k}, \eta, i)$ where $i=1,2$ is the discrete index of the two-component vectors. In Eq. (8) and in the following, we use the convention that repeated coordinates are integrated over as

$O\left(x, x^{\prime}\right) . \psi\left(x^{\prime}\right)=\int \mathrm{d} \boldsymbol{k}^{\prime} \mathrm{d} \eta^{\prime} \sum_{i^{\prime}=1}^{2} O_{i, i^{\prime}}\left(\boldsymbol{k}, \eta ; \boldsymbol{k}^{\prime}, \eta^{\prime}\right) \psi_{i^{\prime}}\left(\boldsymbol{k}^{\prime}, \eta^{\prime}\right)$.

The matrix $O$ reads

$O\left(x, x^{\prime}\right)=\left(\begin{array}{cc}\frac{\partial}{\partial \eta} & -1 \\ -\frac{3 \Omega_{\mathrm{m}}}{2 f^{2}} & \frac{\partial}{\partial \eta}+\frac{3 \Omega_{\mathrm{m}}}{2 f^{2}}-1\end{array}\right) \delta_{\mathrm{D}}\left(\boldsymbol{k}-\boldsymbol{k}^{\prime}\right) \delta_{\mathrm{D}}\left(\eta-\eta^{\prime}\right)$

whereas the symmetric vertex $K_{\mathrm{s}}\left(x ; x_{1}, x_{2}\right)=K_{\mathrm{s}}\left(x ; x_{2}, x_{1}\right)$ writes as

$$
\begin{aligned}
K_{\mathrm{s}}\left(x ; x_{1}, x_{2}\right)= & \delta_{\mathrm{D}}\left(\boldsymbol{k}_{1}+\boldsymbol{k}_{2}-\boldsymbol{k}\right) \delta_{\mathrm{D}}\left(\eta_{1}-\eta\right) \delta_{\mathrm{D}}\left(\eta_{2}-\eta\right) \\
& \times \gamma_{i ; i_{1}, i_{2}}^{\mathrm{s}}\left(\boldsymbol{k}_{1}, \boldsymbol{k}_{2}\right)
\end{aligned}
$$

with

$\gamma_{1 ; 1,2}^{\mathrm{s}}\left(\boldsymbol{k}_{1}, \boldsymbol{k}_{2}\right)=\frac{\left(\boldsymbol{k}_{1}+\boldsymbol{k}_{2}\right) \cdot \boldsymbol{k}_{2}}{2 k_{2}^{2}}$

$\gamma_{1 ; 2,1}^{\mathrm{s}}\left(\boldsymbol{k}_{1}, \boldsymbol{k}_{2}\right)=\frac{\left(\boldsymbol{k}_{1}+\boldsymbol{k}_{2}\right) \cdot \boldsymbol{k}_{1}}{2 k_{1}^{2}}$

$\gamma_{2 ; 2,2}^{\mathrm{s}}\left(\boldsymbol{k}_{1}, \boldsymbol{k}_{2}\right)=\frac{\left|\boldsymbol{k}_{1}+\boldsymbol{k}_{2}\right|^{2}\left(\boldsymbol{k}_{1} \cdot \boldsymbol{k}_{2}\right)}{2 k_{1}^{2} k_{2}^{2}}$,

and zero otherwise (Crocce \& Scoccimarro 2006a; Valageas 2007a). In the following, we use the common approximation

$\frac{\Omega_{\mathrm{m}}}{f^{2}} \simeq 1$,

which simplifies the analysis as the equation of motion (8) no longer shows any explicit dependence on time $\eta$. 


\subsection{Linear regime}

On large scales or at early times where the density and velocity fluctuations are small, one can linearize the equation of motion (8) which yields $O . \psi_{\mathrm{L}}=0$. Then, the linear growing mode is

$\psi_{\mathrm{L}}(x)=\mathrm{e}^{\eta} \delta_{\mathrm{L} 0}(\boldsymbol{k})\left(\begin{array}{l}1 \\ 1\end{array}\right)$

where $\delta_{\mathrm{L} 0}(\boldsymbol{k})$ is the linear density contrast today at redshift $z=0$. As usual, we define the initial conditions by the linear growing mode (16) and we assume Gaussian homogeneous and isotropic initial conditions defined by the linear power spectrum $P_{\mathrm{L} 0}(k)$ :

$\left\langle\delta_{\mathrm{L} 0}\left(\boldsymbol{k}_{1}\right) \delta_{\mathrm{L} 0}\left(\boldsymbol{k}_{2}\right)\right\rangle=P_{\mathrm{L} 0}\left(k_{1}\right) \delta_{\mathrm{D}}\left(\boldsymbol{k}_{1}+\boldsymbol{k}_{2}\right)$.

Then, the linear two-point correlation function $C_{\mathrm{L}}\left(x_{1}, x_{2}\right)$ reads as

$$
\begin{aligned}
C_{\mathrm{L}}\left(x_{1}, x_{2}\right) & =\left\langle\psi_{\mathrm{L}}\left(x_{1}\right) \psi_{\mathrm{L}}\left(x_{2}\right)\right\rangle \\
& =\mathrm{e}^{\eta_{1}+\eta_{2}} P_{\mathrm{L} 0}\left(k_{1}\right) \delta_{\mathrm{D}}\left(\boldsymbol{k}_{1}+\boldsymbol{k}_{2}\right)\left(\begin{array}{ll}
1 & 1 \\
1 & 1
\end{array}\right) .
\end{aligned}
$$

In previous papers (Valageas 2007a,b) we noted the two-point correlation as " $G$ ", but since a restriction of the response function introduced below is also labeled " $G$ " in the literature, we shall note correlation functions as " $C$ " in this article. As in Valageas (2004), it is convenient to introduce the response function $R\left(x_{1}, x_{2}\right)$. Using the approximation (15) it reads in the linear regime (Crocce \& Scoccimarro 2006a; Valageas 2007a)

$$
\begin{aligned}
& R_{\mathrm{L}}\left(x_{1}, x_{2}\right)=\delta_{\mathrm{D}}\left(\boldsymbol{k}_{1}-\boldsymbol{k}_{2}\right) \theta\left(\eta_{1}-\eta_{2}\right) \\
& \quad \times\left\{\frac{\mathrm{e}^{\eta_{1}-\eta_{2}}}{5}\left(\begin{array}{ll}
3 & 2 \\
3 & 2
\end{array}\right)+\frac{\mathrm{e}^{-3\left(\eta_{1}-\eta_{2}\right) / 2}}{5}\left(\begin{array}{cc}
2 & -2 \\
-3 & 3
\end{array}\right)\right\},
\end{aligned}
$$

where the Heaviside factor $\theta\left(\eta_{1}-\eta_{2}\right)$ ensures that causality is satisfied. Thanks to statistical homogeneity and isotropy the correlation functions $C$ (both the nonlinear $C$ as well as $C_{\mathrm{L}}$ ) are symmetric and of the form

$C\left(x_{1}, x_{2}\right)=\delta_{\mathrm{D}}\left(\boldsymbol{k}_{1}+\boldsymbol{k}_{2}\right) C_{i_{1}, i_{2}}\left(k_{1} ; \eta_{1}, \eta_{2}\right)$,

with

$C_{i_{1}, i_{2}}\left(k ; \eta_{1}, \eta_{2}\right)=C_{i_{2}, i_{1}}\left(k ; \eta_{2}, \eta_{1}\right)$,

whereas the response functions $R$ (again both the nonlinear $R$ as well as $R_{\mathrm{L}}$ ) are of the form

$R\left(x_{1}, x_{2}\right)=\delta_{\mathrm{D}}\left(\boldsymbol{k}_{1}-\boldsymbol{k}_{2}\right) \theta\left(\eta_{1}-\eta_{2}\right) R_{i_{1}, i_{2}}\left(k_{1} ; \eta_{1}, \eta_{2}\right)$.

Moreover, the linear two-point functions obey (Valageas 2007a,b):

$O(x, z) \cdot C_{\mathrm{L}}(z, y)=0, \quad O(x, z) \cdot R_{\mathrm{L}}(z, y)=\delta_{\mathrm{D}}(x-y)$.

This can also be checked from expressions (18), (19).

\subsection{Path-integral formalism and large- $N$ expansions}

As described in Valageas $(2007 a, b)$, the statistical properties of the density and velocity fields can be obtained from the generating functional

$Z[j]=\left\langle\mathrm{e}^{j \cdot \psi}\right\rangle=\int[\mathrm{d} \psi][\mathrm{d} \lambda] \mathrm{e}^{j \cdot \psi-S[\psi, \lambda]}$, where we have introduced an imaginary auxiliary field $\lambda(x)$, and the action $S[\psi, \lambda]$ reads as ${ }^{1}$

$S[\psi, \lambda]=\lambda \cdot\left(O \cdot \psi-K_{\mathrm{s}} \cdot \psi \psi\right)-\frac{1}{2} \lambda \cdot \Delta_{\mathrm{I}} \cdot \lambda$,

where $\Delta_{\mathrm{I}}$ is the two-point correlation of the initial conditions taken at time $\eta_{\mathrm{I}}$. This matrix disappears in the final equations when we take the limit $\eta_{\mathrm{I}} \rightarrow-\infty$. Moreover, the first and second moments of the field $\lambda$ obey

$\langle\lambda\rangle=0, \quad\langle\lambda \lambda\rangle=0, \quad\left\langle\psi\left(x_{1}\right) \lambda\left(x_{2}\right)\right\rangle=R\left(x_{1}, x_{2}\right)$.

The action (25) can serve as a basis for several approaches borrowed from field theory. For instance, it was used in Matarrese \& Pietroni $(2007 \mathrm{a}, \mathrm{b})$ to derive a resummation scheme based on the dependence of the system on a high- $k$ cutoff (see also Valageas 2007b, for a discussion of this approach in the case of the Zeldovich dynamics). Another approach relies on standard "large- $N$ " expansion schemes (Valageas 2007a,b). Thus, generalizing the problem to $N$ fields or simply multiplying the exponent in expression (24) by a factor $N$, one can derive various expansions over powers of $1 / N$ (which agree up to the order $1 / N^{q}$ of the expansion but differ by higher-order terms as they involve different partial resummations). This path-integral formalism can also be written for the integral form of the equation of motion, as discussed in Valageas (2007b).

\subsection{Direct steepest-descent method}

A first large- $N$ expansion is provided by a direct steepest-descent method, which expands the path integral (24) around a saddlepoint as described in Valageas $(2004,2007 a)$. It introduces auxiliary correlation and response functions $C_{0}$ and $R_{0}$ defined by evolution equations that happen to be identical to the linearized Eq. (23). Thus, we have $C_{0}=C_{\mathrm{L}}$ and $R_{0}=R_{\mathrm{L}}$. (This is specific to the hydrodynamic description, where $\langle\psi\rangle=0$, and does not hold if we use the Vlasov equation of motion to describe the system.) Then, the nonlinear correlation and response functions $C$ and $R$ can be obtained from the coupled linear equations

$O(x, z) \cdot C(z, y)=\Sigma(x, z) \cdot C(z, y)+\Pi(x, z) \cdot R^{\mathrm{T}}(z, y)$

$O(x, z) \cdot R(z, y)=\delta_{\mathrm{D}}(x-y)+\Sigma(x, z) \cdot R(z, y)$

where we have introduced the self-energy matrices $\Sigma$ and $\Pi$ that can be expressed in terms of $C_{0}$ and $R_{0}$ (hence in terms of $C_{\mathrm{L}}$ and $R_{\mathrm{L}}$ ). Equations $(27)-(28)$ are actually exact (they may be seen as the definition of the self-energy matrices) and the order $1 / N^{\mathrm{q}}$ of the large- $N$ expansion only enters the expression of the self-energy, which can be obtained from a series of diagrams. At one-loop order, there is only one contribution which reads as

$\Sigma(x, y)=4 K_{\mathrm{s}}\left(x ; x_{1}, x_{2}\right) K_{\mathrm{s}}\left(z ; y, z_{2}\right) R_{\mathrm{L}}\left(x_{1}, z\right) C_{\mathrm{L}}\left(x_{2}, z_{2}\right)$

$\Pi(x, y)=2 K_{s}\left(x ; x_{1}, x_{2}\right) K_{\mathrm{s}}\left(y ; y_{1}, y_{2}\right) C_{\mathrm{L}}\left(x_{1}, y_{1}\right) C_{\mathrm{L}}\left(x_{2}, y_{2}\right)$.

\subsection{The 2PI effective action method}

A second large- $N$ expansion is provided by a 2PI effective action approach that involves a diagrammatic expansion of the double Legendre transform $\Gamma[\psi, C]$ of the generating functional of connected correlation functions (Valageas 2007a). This is also the

\footnotetext{
1 Note that the action $S$ only takes such a simple form because the Jacobian, associated with the change of variables from the initial condition $\psi_{\mathrm{L}}$ to the nonlinear field $\psi$, can be shown to be an irrelevant constant.
} 
generating functional of two-particle irreducible (2PI) diagrams, that is those that cannot be disconnected by cutting two lines. This approach yields the same Schwinger-Dyson Eqs. (27), (28), but the self-energy is now given in terms of the nonlinear twopoint functions $C$ and $R$. Therefore, this method does not introduce the auxiliary functions $C_{0}$ and $R_{0}$, but the system (27), (28) is now a pair of nonlinear coupled equations $(\Sigma \propto R C$ and $\Pi \propto C C$ at one-loop order). As shown in Valageas (2007a), this nonlinearity is the key to the small-scale damping of the nonlinear response $R$ (at one-loop order). At one-loop order, the selfenergy has the same structure as for the direct steepest-descent method and it reads as

$$
\begin{aligned}
\Sigma(x, y) & =4 K_{\mathrm{s}}\left(x ; x_{1}, x_{2}\right) K_{\mathrm{s}}\left(z ; y, z_{2}\right) R\left(x_{1}, z\right) C\left(x_{2}, z_{2}\right) \\
\Pi(x, y) & =2 K_{\mathrm{s}}\left(x ; x_{1}, x_{2}\right) K_{\mathrm{s}}\left(y ; y_{1}, y_{2}\right) C\left(x_{1}, y_{1}\right) C\left(x_{2}, y_{2}\right) .
\end{aligned}
$$

Thus, Eqs. (31), (32) are equal to Eqs. (29), (30) where the linear two-point functions are replaced by the nonlinear ones. This simple correspondence would not hold at higher orders as the selfenergies associated with the steepest-descent approach would involve additional terms as compared with the $2 \mathrm{PI}$ effective action approach.

Approximation schemes based on equations of the form (27), (28), (31), (32), have been applied to many fields of theoretical physics, such as quantum field theory (e.g. Zinn-Justin 1989; Berges 2002). They can be derived in different manners and are also called "mode-coupling approximations", in condensed matter and studies of glassy systems (Bouchaud et al. 1996), or "direct interaction approximation" in studies of turbulent flows (Kraichnan 1961). A derivation inspired from works on turbulence is presented in Taruya \& Hiramatsu (2007). An advantage of the derivation from the path integral (24) is its relative simplicity and the well-known diagrammatics associated with the expansion up to high orders. Moreover, it satisfies an exact variational principle (the equations above are associated with the saddle-point of the 2PI effective action $\Gamma$ and it is this action which is approximated by truncating an expansion over $1 / N$, see Valageas 2004). Although we do not use this property here, it might prove useful at some point. On the other hand, the path integral (24) may serve as a basis for other approximation schemes.

\subsection{Comparison between various approaches}

The standard Schwinger-Dyson Eqs. (27), (28) can also be obtained in a diagrammatic fashion from the 1PI effective action $\Gamma[\psi]$, also called the generating functional of proper vertices (see any textbook on quantum field theory). It is the generating functional of one-particle irreducible (1PI) diagrams, that is those that cannot be disconnected by cutting one line, and it is also the Legendre transform of $W=\ln Z$, where $Z$ is given by Eq. (24). Thus, Scoccimarro \& Crocce (2006a) describe a diagrammatic derivation of Eqs. (27), (28), starting from the equation of motion (8) written in integral form (as in Eq. (79) below).

As noticed above, Eqs. (27), (28) alone are mostly a rewriting of the problem, and one still needs to specify a method to compute the self-energies $\Sigma$ and $\Pi$. We have described above two such methods, the direct steepest-descent approach, where the self-energy is written in terms of the linear two-point functions $C_{\mathrm{L}}$ and $R_{\mathrm{L}}$, and the 2 PI effective action approach, where the self-energy is written in terms of the nonlinear two-point functions $C$ and $R$. Of course, both methods can also be obtained by diagrammatic means. For instance, Scoccimarro \& Crocce (2006a) present a loopwise expansion in terms of the nonlinear two-point functions that coincides with the 2PI effective action approach (see also L'vov \& Procaccia 1995, for a detailed description of such a diagrammatic procedure).

Next, it is possible to reexpress the self-energy in a more compact fashion by introducing "dressed vertices" that go beyond the tree-order vertex $K_{\mathrm{s}}$. That is, one can recognize threepoint diagrams such as those of Fig. 10 (but written in terms of nonlinear two-point functions) in parts of larger diagrams, and identify them with the expansion of properly defined dressed vertices (L'vov \& Procaccia 1995). However, this leads to another expansion scheme than the strict 2PI scheme, that would be more closely related to the 1PI effective action approach.

Indeed, looking for an expansion of the self-energies $\Sigma$ and $\Pi$ in terms of the nonlinear $R$ and $C$ does not uniquely specify the practical procedure used to compute correlation functions. One can always reorganize the diagrammatic series in different fashions that lead to different truncations. For instance, as discussed in Sect. 5 of Valageas (2004), and recalled above, it is possible to derive the Schwinger-Dyson Eqs. (27), (28) from the 1PI effective action $\Gamma[\psi]$. Then, the self-energy is given by an exact expression that involves the proper vertex $\Gamma_{3}$ which "renormalizes" the bare vertex $K_{\mathrm{s}}$ (its first two terms are given in Eqs. (70), (76) below). Next, the proper vertices $\Gamma_{\mathrm{q}}$ obey a hierarchy of equations, that must be truncated at some order $q$ (just like the familiar BBGKY hierarchy obeyed by the correlations $C_{\mathrm{q}}$ can be truncated at some order to build an approximation scheme in the weakly nonlinear regime). This approach provides another expansion scheme, written in terms of nonlinear two-point functions, that also contains infinite partial resummations as compared with the standard perturbative expansion recalled in Sect. 3 below.

For completeness, let us point out that we always consider the limit $\eta_{\mathrm{I}} \rightarrow-\infty$ (i.e. the initial conditions are set up at a redshift $\left.z_{\mathrm{I}} \rightarrow \infty\right)$ and the response function $R\left(x_{1}, x_{2}\right)$ is defined as the deviation from the mean of the fields at time $\eta_{1}$ with respect to a perturbation at an arbitrary earlier time $\eta_{2}$. By contrast, the calculations presented in Scoccimarro \& Crocce (2006a,b) consider the system defined by initial conditions set at a finite time $\eta_{\mathrm{I}}$ (which defines the origin of time as $\eta_{\mathrm{I}}=0$ ) and focus on the propagator $G$ defined from the restriction of $R\left(x_{1}, x_{2}\right)$ to $\eta_{2}=\eta_{\mathrm{I}}$. However, the formalism of Scoccimarro \& Crocce (2006a,b) can also be applied to the case $\eta_{\mathrm{I}} \rightarrow-\infty$ (e.g. Sect. 6 below).

\subsection{Zeldovich dynamics}

The gravitational dynamics described in the previous sections is rather complex and analytical computations often lead to complicated expressions. Therefore, it can be interesting to investigate a closely related dynamics that obeys a similar equation of motion but has a simpler structure. The Zeldovich dynamics offers such an opportunity (Zeldovich 1970; Gurbatov et al. 1989). As described in Valageas (2007b), this dynamics can also be defined from the hydrodynamical Eqs. (1), (2), where the velocity potential is used in place of the gravitational potential. Then, the equation of motion again has the form (8). The quadratic vertex $K_{\mathrm{s}}$ is unchanged whereas the linear operator $O$ is changed to

$O\left(x, x^{\prime}\right)=\left(\begin{array}{cc}\frac{\partial}{\partial \eta} & -1 \\ 0 & \frac{\partial}{\partial \eta}-1\end{array}\right) \delta_{\mathrm{D}}\left(\boldsymbol{k}-\boldsymbol{k}^{\prime}\right) \delta_{\mathrm{D}}\left(\eta-\eta^{\prime}\right)$

This yields the same linear growing mode (16) hence the same linear two-point correlation (18). However, the linear decaying mode is modified by the change of $O$; this also modifies the 
linear response as

$$
\begin{array}{r}
R_{\mathrm{L}}\left(x_{1}, x_{2}\right)=\delta_{\mathrm{D}}\left(\boldsymbol{k}_{1}-\boldsymbol{k}_{2}\right) \theta\left(\eta_{1}-\eta_{2}\right) \\
\times\left\{\mathrm{e}^{\eta_{1}-\eta_{2}}\left(\begin{array}{ll}
0 & 1 \\
0 & 1
\end{array}\right)+\left(\begin{array}{cc}
1 & -1 \\
0 & 0
\end{array}\right)\right\} .
\end{array}
$$

Then, the path-integral formalism of Sect. 2.3 can be applied in exactly the same manner and we obtain the same action (25), where we must only substitute the new linear operator $O$ (Valageas 2007b). Therefore, large- $N$ expansions again lead to Eqs. (27)-(32). Thus, the Eulerian equations of motion have the same structure and any expansion scheme can be applied in identical manner to both dynamics. However, in Lagrangian space the Zeldovich dynamics is much simpler as its solution is given by

$\boldsymbol{x}=\boldsymbol{q}+D_{+}(\eta) \boldsymbol{s}_{\mathrm{L} 0}(\boldsymbol{q})$,

where $\boldsymbol{q}$ is the Lagrangian coordinate of the particle. That is, the trajectories of particles are given by the linear displacement field $s_{\mathrm{L}}=D_{+} s_{\mathrm{L} 0}$. Going back to Eulerian space, one can derive from the solution (35) an explicit expression for the matter density contrast. Starting from the uniform matter density $\bar{\rho}$ at $t \rightarrow 0$, the conservation of matter gives, before orbit-crossing,

$\rho(\boldsymbol{x}) \mathrm{d} \boldsymbol{x}=\bar{\rho} \mathrm{d} \boldsymbol{q} \quad$ whence $1+\delta(\boldsymbol{x})=\left|\operatorname{det}\left(\frac{\partial \boldsymbol{x}}{\partial \boldsymbol{q}}\right)\right|^{-1}$.

This also reads from Eq. (35) (Schneider \& Bartelmann 1995; Taylor \& Hamilton 1996) as

$\delta(\boldsymbol{x}, \eta)=\int \mathrm{d} \boldsymbol{q} \delta_{D}\left[\boldsymbol{x}-\boldsymbol{q}-\boldsymbol{s}_{\mathrm{L}}(\boldsymbol{q}, \eta)\right]-1$.

In Fourier space we obtain

$\delta(\boldsymbol{k}, \eta)=\int \frac{\mathrm{d} \boldsymbol{q}}{(2 \pi)^{3}} \mathrm{e}^{-\mathrm{i} \boldsymbol{k} \cdot \boldsymbol{q}}\left[\mathrm{e}^{-\mathrm{i} \boldsymbol{k} \cdot s_{L}(\boldsymbol{q}, \eta)}-1\right]$,

whereas the linear displacement field is related to the linear density contrast by

$\nabla_{\boldsymbol{q}} \cdot \boldsymbol{s}_{\mathrm{L}}=-\delta_{\mathrm{L}}, \quad \boldsymbol{s}_{\mathrm{L}}(\boldsymbol{k}, \eta)=i \frac{\boldsymbol{k}}{k^{2}} \delta_{\mathrm{L}}(\boldsymbol{k}, \eta)$.

From Eq. (38) one can also derive the exact expressions of the nonlinear correlation $C$ and response $R$ (Schneider \& Bartelmann 1995; Taylor \& Hamilton 1996; Valageas 2007b).

\section{Standard perturbation theory}

In this section, we recall the standard perturbation theory applied to the cosmological gravitational dynamics (Fry 1984; Goroff et al. 1986; Bernardeau et al. 2002; Scoccimarro 1997). As explained in Sect. 2.7, note that all expressions derived below apply as well to the Zeldovich dynamics. In this approach, one expands the density and velocity fields over powers of the linear growing mode $(16)$ as $\left(\psi_{1}=\delta\right)$

$$
\begin{aligned}
\delta(\boldsymbol{k}, D)= & \sum_{n=1}^{\infty} D^{n} \int \mathrm{d} \boldsymbol{q}_{1} . . \boldsymbol{q}_{n} \delta_{\mathrm{D}}\left(\boldsymbol{q}_{1}+. .+\boldsymbol{q}_{n}-\boldsymbol{k}\right) \\
& \times F_{n}\left(\boldsymbol{q}_{1}, . ., \boldsymbol{q}_{n}\right) \delta_{\mathrm{L} 0}\left(\boldsymbol{q}_{1}\right) . . \delta_{\mathrm{L} 0}\left(\boldsymbol{q}_{n}\right),
\end{aligned}
$$

and $\left(\psi_{2}=-\nabla . v / \mathcal{H} f\right)$

$$
\begin{aligned}
\psi_{2}(\boldsymbol{k}, D)= & \sum_{n=1}^{\infty} D^{n} \int \mathrm{d} \boldsymbol{q}_{1} . . \boldsymbol{q}_{n} \delta_{\mathrm{D}}\left(\boldsymbol{q}_{1}+. .+\boldsymbol{q}_{n}-\boldsymbol{k}\right) \\
& \times E_{n}\left(\boldsymbol{q}_{1}, . ., \boldsymbol{q}_{n}\right) \delta_{\mathrm{L} 0}\left(\boldsymbol{q}_{1}\right) . . \delta_{\mathrm{L} 0}\left(\boldsymbol{q}_{n}\right),
\end{aligned}
$$

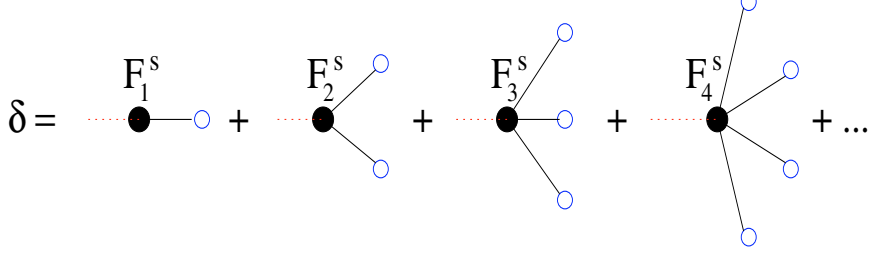

Fig. 1. The diagrams associated with the standard perturbative expansion (40) of the density field $\delta(\boldsymbol{k}, \eta)$ over powers of the linear growing mode $\delta_{\mathrm{L}}$. The filled circles are the vertices $F_{n}^{s}$ attached to $n$ linear modes $\delta_{\mathrm{L}}$ shown by the open circles. The left dotted legs are attached to the external wavenumber $\boldsymbol{k}$ of the nonlinear density $\delta(\boldsymbol{k}, \eta)$.

with $F_{1}=E_{1}=1$ (in Eqs. (40)-(41) and in the following $\boldsymbol{q}_{j}$ is not a Lagrangian coordinate but a wavenumber associated with a linear growing mode). Here (and in most cases below) we use the linear growth factor $D=\mathrm{e}^{\eta}$ of Eq. (5) as the time coordinate. The factorization of the time dependence as $D^{n}$ is due to the approximation $\Omega_{\mathrm{m}} / f^{2} \simeq 1$ (and it is exact for the Einstein-de Sitter cosmology). Then, by substituting the expansions (40), (41) into the equation of motion (8) one obtains a recursion relation between $\left(F_{n}, E_{n}\right)$ and $\left(F_{n+1}, E_{n+1}\right)$. This enables one to compute the kernels $F_{n}$ and the density and velocity fields up to the required order. Moreover, one can easily check that the kernels $F_{n}$ and $E_{n}$ obey the symmetry $F_{n}\left(-\boldsymbol{q}_{1}, . .,-\boldsymbol{q}_{n}\right)=F_{n}\left(\boldsymbol{q}_{1}, . ., \boldsymbol{q}_{n}\right)$ since $\delta(\boldsymbol{x})$ and $\theta(\boldsymbol{x})$ are real (whence $\left.\delta(-\boldsymbol{k})=\delta(\boldsymbol{k})^{*}\right)$. They also satisfy $F_{n}\left(\boldsymbol{q}_{1}, . ., \boldsymbol{q}_{n}\right)=0$ for $\boldsymbol{q}_{1}+. .+\boldsymbol{q}_{n}=0$ as $\delta(\boldsymbol{k}=0)=0$ at all times because of the conservation of matter. It is convenient to introduce the symmetric vertices $F_{n}^{s}$ obtained by summing over all permutations of $\left(\boldsymbol{q}_{1}, . ., \boldsymbol{q}_{n}\right)$ as

$F_{n}^{s}\left(\boldsymbol{q}_{1}, . ., \boldsymbol{q}_{n}\right)=\frac{1}{n !} \sum_{\text {perm. }} F_{n}\left(\boldsymbol{q}_{1}, . ., \boldsymbol{q}_{n}\right)$.

The diagrammatic expansion associated with (40) is shown in Fig. 1. The diagrams are very simple to draw but the complexity is hidden in the vertices $F_{n}^{s}$ that are obtained by recursion from lower-order vertices. Note that one needs to compute a new vertex at each order of the expansion.

Then, from the expansion (40), one obtains for any correlation function a perturbative expansion over powers of the linear power spectrum $P_{\mathrm{L}}$ by using Wick's theorem. In particular, the two-point density correlation $C_{\delta 2}$ reads (up to order $\delta_{\mathrm{L}}^{4}$ whence up to order $P_{\mathrm{L}}^{2}$ ) as

$$
\begin{aligned}
C_{\delta 2}=\langle\delta \delta\rangle_{c}= & \left\langle\delta^{(1)} \delta^{(1)}\right\rangle+\left\langle\delta^{(3)} \delta^{(1)}\right\rangle+\left\langle\delta^{(1)} \delta^{(3)}\right\rangle \\
& +\left\langle\delta^{(2)} \delta^{(2)}\right\rangle+\ldots,
\end{aligned}
$$

where $\delta^{(n)} \propto F_{n}^{s} \delta_{\mathrm{L}}^{n}$ is the term of order $n$ in the expansion (40). The diagrammatic expansion associated with Eq. (43) is displayed in Fig. 2. The order of the diagrams over $P_{\mathrm{L}}$ scales with the number of loops $\ell$ as $P_{\mathrm{L}}^{\ell+1}$. Up to order $P_{\mathrm{L}}^{2}$, we can write the equal-time nonlinear power spectrum $P(k)$ as

$P(k)=P^{\text {tree }}(k)+P^{1 \text { loop }}(k)$,

with

$P^{\text {tree }}=P^{(a)}=P_{\mathrm{L}}(k), \quad P^{1 \text { loop }}=P^{(b)}+P^{(c)}$.

Thus, the tree diagram (a) of Fig. 2 is merely equal to the linear power spectrum $P_{\mathrm{L}}$ whereas the one-loop contributions $P^{(b)}$ and $P^{(c)}$ of diagrams (b) and (c) are given by

$P^{(b)}(k)=6 P_{\mathrm{L}}(k) \int \mathrm{d} \boldsymbol{q} P_{\mathrm{L}}(q) F_{3}^{s}(\boldsymbol{q},-\boldsymbol{q}, \boldsymbol{k})$, 
(a)

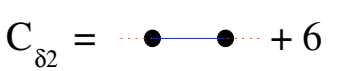

(c) $+2$

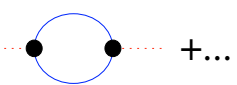

Fig. 2. The diagrams associated with the standard perturbative expansion (43) of the two-point density correlation $C_{\delta 2}$ over powers of the linear power spectrum $P_{\mathrm{L}}$. The big dots are the vertices $F_{n}^{s}$ and the solid lines are the linear power spectrum $P_{\mathrm{L}}$. We also give the overall multiplicity factor of each diagram, up to one-loop order (i.e. $P_{\mathrm{L}}^{2}$ for $C_{\delta 2}$ ).

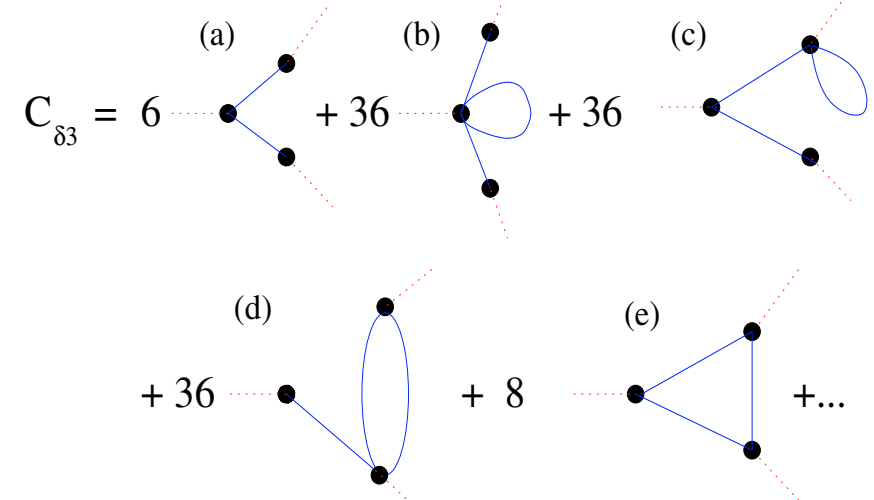

Fig. 3. The diagrams associated with the standard perturbative expansion (48) of the three-point density correlation $C_{\delta 3}$ over powers of the linear power spectrum $P_{\mathrm{L}}$ (shown up to one-loop order, i.e. $P_{\mathrm{L}}^{3}$ for $C_{\delta 3}$ ). The symbols are as in Fig. 2.

$$
P^{(c)}(k)=2 \int \mathrm{d} \boldsymbol{q} P_{\mathrm{L}}(q) P_{\mathrm{L}}(|\boldsymbol{k}-\boldsymbol{q}|) F_{2}^{s}(\boldsymbol{q}, \boldsymbol{k}-\boldsymbol{q})^{2} .
$$

We give in Fig. 2 the overall multiplicity factor of each diagram. Thus, the factor 6 for diagram (b) in Fig. 2 reads as $6=2 \times 3$, where the factor 2 comes from Eq. (43) (associated with the two contributions $\left.\left\langle\delta^{(3)} \delta^{(1)}\right\rangle+\left\langle\delta^{(1)} \delta^{(3)}\right\rangle\right)$ whereas the factor 3 comes from the three possible Gaussian pairings which can be built for each term $\left\langle\delta^{(3)} \delta^{(1)}\right\rangle$ (Wick's theorem). In this article, we focus on equal-time statistics, such as the usual matter power spectrum $P(k)$, and each factor of $P_{\mathrm{L}}$ gives rise to a time-dependent factor $D^{2}$, where $D$ is the linear growth factor at the time of interest, but the analysis applies as well to different-time correlations.

On the other hand, the three-point density correlation $C_{\delta 3}$ reads (up to order $P_{\mathrm{L}}^{3}$ ) as

$$
\begin{aligned}
C_{\delta 3}= & \left\langle\delta^{(2)} \delta^{(1)} \delta^{(1)}\right\rangle+2 \text { perm. }+\left\langle\delta^{(4)} \delta^{(1)} \delta^{(1)}\right\rangle+2 \text { perm. } \\
& +\left\langle\delta^{(3)} \delta^{(2)} \delta^{(1)}\right\rangle+5 \text { perm. }+\left\langle\delta^{(2)} \delta^{(2)} \delta^{(2)}\right\rangle+\ldots,
\end{aligned}
$$

where each term " $n$ perm." stands for $n$ terms obtained by permutations over the previous term, such as

$$
\begin{aligned}
\left\langle\delta^{(2)} \delta^{(1)} \delta^{(1)}\right\rangle+2 \text { perm. }= & \left\langle\delta^{(2)} \delta^{(1)} \delta^{(1)}\right\rangle+\left\langle\delta^{(1)} \delta^{(2)} \delta^{(1)}\right\rangle \\
& +\left\langle\delta^{(1)} \delta^{(1)} \delta^{(2)}\right\rangle .
\end{aligned}
$$

The associated diagrams are shown in Fig. 3. For the equal-time bispectrum $B$ defined by

$C_{\delta 3}\left(\boldsymbol{k}_{1}, \boldsymbol{k}_{2}, \boldsymbol{k}_{3} ; \eta\right)=\delta_{\mathrm{D}}\left(\boldsymbol{k}_{1}+\boldsymbol{k}_{2}+\boldsymbol{k}_{2}\right) B\left(k_{1}, k_{2}, k_{3} ; \eta\right)$,

we obtain at tree-order the well-known result

$B^{\text {tree }}\left(k_{1}, k_{2}, k_{3}\right)=2 P_{\mathrm{L}}\left(k_{2}\right) P_{\mathrm{L}}\left(k_{3}\right) F_{2}^{s}\left(\boldsymbol{k}_{2}, \boldsymbol{k}_{3}\right)+2$ perm.

and at one-loop order:

$B^{1 \text { loop }}\left(k_{1}, k_{2}, k_{3}\right)=B^{(b)}+B^{(c)}+B^{(d)}+B^{(e)}$,

with (Scoccimarro 1997):

$$
\begin{aligned}
B^{(b)}= & 12 P_{\mathrm{L}}\left(k_{2}\right) P_{\mathrm{L}}\left(k_{3}\right) \int \mathrm{d} \boldsymbol{q} P_{\mathrm{L}}(q) F_{4}^{s}\left(-\boldsymbol{q}, \boldsymbol{q}, \boldsymbol{k}_{2}, \boldsymbol{k}_{3}\right) \\
& +2 \text { perm., } \\
B^{(c)}= & 6 P_{\mathrm{L}}\left(k_{2}\right) P_{\mathrm{L}}\left(k_{3}\right) F_{2}^{s}\left(\boldsymbol{k}_{2}, \boldsymbol{k}_{3}\right) \int \mathrm{d} \boldsymbol{q} P_{\mathrm{L}}(q) \\
& \times F_{3}^{s}\left(\boldsymbol{q},-\boldsymbol{q}, \boldsymbol{k}_{2}\right)+5 \text { perm. }
\end{aligned}
$$

$$
\begin{aligned}
B^{(d)}= & 6 P_{\mathrm{L}}\left(k_{1}\right) \int \mathrm{d} \boldsymbol{q}_{1} \mathrm{~d} \boldsymbol{q}_{2} \delta_{\mathrm{D}}\left(\boldsymbol{q}_{1}+\boldsymbol{q}_{2}-\boldsymbol{k}_{2}\right) P_{\mathrm{L}}\left(q_{1}\right) \\
\times & P_{\mathrm{L}}\left(q_{2}\right) F_{2}^{s}\left(\boldsymbol{q}_{1}, \boldsymbol{q}_{2}\right) F_{3}^{s}\left(\boldsymbol{q}_{1}, \boldsymbol{q}_{2}, \boldsymbol{k}_{1}\right)+5 \text { perm. }, \\
B^{(e)}= & 8 \int \mathrm{d} \boldsymbol{q}_{1} \mathrm{~d} \boldsymbol{q}_{2} \mathrm{~d} \boldsymbol{q}_{3} \delta_{\mathrm{D}}\left(\boldsymbol{q}_{1}+\boldsymbol{q}_{2}-\boldsymbol{k}_{1}\right) \\
& \times \delta_{\mathrm{D}}\left(\boldsymbol{q}_{1}+\boldsymbol{q}_{3}+\boldsymbol{k}_{2}\right) P_{\mathrm{L}}\left(q_{1}\right) P_{\mathrm{L}}\left(q_{2}\right) P_{\mathrm{L}}\left(q_{3}\right) \\
& \times F_{2}^{s}\left(\boldsymbol{q}_{1}, \boldsymbol{q}_{2}\right) F_{2}^{s}\left(\boldsymbol{q}_{1}, \boldsymbol{q}_{3}\right) F_{2}^{s}\left(-\boldsymbol{q}_{2}, \boldsymbol{q}_{3}\right) .
\end{aligned}
$$

The derivation of higher-order correlations proceeds in exactly the same manner, by writing $C_{\delta p}=\left\langle\delta_{1} . . \delta_{p}\right\rangle$, expanding each factor $\delta_{j}$ through Eq. (40), and performing the Gaussian average with Wick's theorem.

\section{Expansion over cubic interaction term}

In this section, we recall how to recover the results of the standard perturbation theory from the path integral (24). As described in Valageas $(2004,2007 a)$, the usual perturbative expansion presented in Sect. 3 over powers of the linear growing mode $\delta_{\mathrm{L}}$ can also be seen as an expansion over powers of the interaction vertex $K_{\mathrm{s}}$ of the equation of motion (8). (Formally one may add a coupling constant $g$ as $K_{\mathrm{s}} \rightarrow g K_{\mathrm{s}}$ and expand over powers of $g$.) Indeed, by substituting the expansions (40), (41) into the equation of motion (8) to derive the recursion relation between $\left(F_{n}, E_{n}\right)$ and $\left(F_{n+1}, E_{n+1}\right)$, we can see that $F_{n} \propto K_{\mathrm{s}}^{n-1}$ whence $\delta^{(n)} \propto K_{\mathrm{s}}^{n-1}$ (by which we actually mean $\delta^{(n)} \propto g^{n-1}$ ). Therefore, the expansion over powers of $\delta_{\mathrm{L}}$, or over powers of $P_{\mathrm{L}}$ for averaged quantities such as correlation functions, is identical to the expansion over powers of $K_{\mathrm{s}}$. Then, this latter expansion can be directly obtained from the path integral (24) by expanding over the cubic part $\lambda . K_{\mathrm{s}} . \psi \psi$ of the action $S[\psi, \lambda]$ of Eq. (25). This equivalence applies to all higher-order correlation functions.

Thus, we obtain for the two-point function

$$
\begin{aligned}
C_{2}\left(x_{1}, x_{2}\right) & =\left\langle\psi\left(x_{1}\right) \psi\left(x_{2}\right)\right\rangle=\int[\mathrm{d} \psi][\mathrm{d} \lambda] \psi\left(x_{1}\right) \psi\left(x_{2}\right) \mathrm{e}^{-S[\psi, \lambda]} \\
= & \int[\mathrm{d} \psi][\mathrm{d} \lambda] \psi\left(x_{1}\right) \psi\left(x_{2}\right)\left(1+\lambda K_{\mathrm{s}} \psi^{2}+\frac{\left(\lambda K_{\mathrm{s}} \psi^{2}\right)^{2}}{2}+. .\right) \mathrm{e}^{-S_{0}} \\
= & \left\langle\psi\left(x_{1}\right) \psi\left(x_{2}\right)\left(1+\lambda K_{\mathrm{s}} \psi^{2}+\frac{\left(\lambda K_{\mathrm{s}} \psi^{2}\right)^{2}}{2}+. .\right)\right\rangle_{0}
\end{aligned}
$$

where $S_{0}=\lambda . O . \psi-\frac{1}{2} \lambda . \Delta_{\mathrm{I}} \cdot \lambda$ is the quadratic part of the action $S[\psi, \lambda]$ and $\langle. .\rangle_{0}$ is the average with respect to this Gaussian action $S_{0}$. Then, one can use Wick's theorem to compute the Gaussian path integral defined by $S_{0}$. Since the action $S_{0}$ is equal to the action $S$ with $K_{\mathrm{s}}=0$, it actually corresponds to the linear dynamics. Thus we have (as can also be explicitly checked):

$$
\begin{gathered}
\langle\psi\rangle_{0}=\langle\lambda\rangle_{0}=0, \quad\left\langle\psi\left(x_{1}\right) \psi\left(x_{2}\right)\right\rangle_{0}=C_{\mathrm{L}}\left(x_{1}, x_{2}\right), \\
\left\langle\psi\left(x_{1}\right) \lambda\left(x_{2}\right)\right\rangle_{0}=R_{\mathrm{L}}\left(x_{1}, x_{2}\right), \quad\left\langle\lambda\left(x_{1}\right) \lambda\left(x_{2}\right)\right\rangle_{0}=0 .
\end{gathered}
$$




$$
\mathrm{C}_{\mathrm{L}}\left(\mathrm{x}_{1}, \mathrm{x}_{2}\right)=\mathrm{x}_{1}-\mathrm{x}_{2}, \quad \mathrm{R}_{\mathrm{L}}\left(\mathrm{x}_{1}, \mathrm{x}_{2}\right)=\mathrm{x}_{1}-\mathrm{x}_{2}
$$

Fig. 4. The diagrammatic symbols used for the two-point functions $C_{\mathrm{L}}=\langle\psi \psi\rangle_{0}$ and $R_{\mathrm{L}}=\langle\psi \lambda\rangle_{0}$. The third possible two-point function vanishes: $\langle\lambda \lambda\rangle_{0}=0$. The propagator $R_{\mathrm{L}}=\left\langle\psi\left(x_{1}\right) \lambda\left(x_{2}\right)\right\rangle_{0}$ is distinguished from $C_{\mathrm{L}}$ by the addition of an arrow which marks the direction of propagation, from the response-field $\lambda\left(x_{2}\right)$ towards the physical field $\psi\left(x_{1}\right)$, that is, from time $D_{2}$ to time $D_{1}>D_{2}$ as enforced by the Heaviside factor of Eq. (19). (This is due to causality.)

In terms of diagrams, we note these linear propagators as in Fig. 4. We add an arrow to the lines associated with the propagator $R_{\mathrm{L}}=\left\langle\psi\left(x_{1}\right) \lambda\left(x_{2}\right)\right\rangle_{0}$ to mark the arrow of time due to causality $\left(D_{1}>D_{2}\right)$, which is enforced by the Heaviside factor of Eq. (19). Thus, the response $R_{\mathrm{L}}$ runs from the response-field $\lambda$ towards the physical field $\psi$. We could have added an out-going arrow at both ends of $C_{\mathrm{L}}$ (both ends are associated with fields $\psi$ ) but for simplicity we put no arrow at all. Since each vertex $K_{\mathrm{s}}$ is associated with one response-field $\lambda$ and two physical fields $\psi$, through $\lambda K_{\mathrm{s}} \psi \psi$ as in the action (25), each vertex $K_{\mathrm{s}}$ shown by a big dot in Fig. 5 and hereafter must be connected to one outgoing line (hence a response $R_{\mathrm{L}}$ ) and to two incoming lines (which can be $R_{\mathrm{L}}$ or $C_{\mathrm{L}}$ with the understanding that $C_{\mathrm{L}}$ can be read with two outgoing lines, that is, one at each end).

Then, from Eq. (57) the two-point correlation reads up to one-loop order as

$C_{2}=C_{2}^{\text {tree }}+C_{2}^{1 \text { loop }}+\ldots$, with $C_{2}^{\text {tree }}=C_{\mathrm{L}}$,

and

$$
\begin{aligned}
C_{2}^{1 \text { loop }}= & \frac{1}{2}\left\langle\psi\left(x_{1}\right) \psi\left(x_{2}\right)\left(\lambda K_{\mathrm{s}} \psi \psi\right)^{2}\right\rangle_{0} \\
= & 8 R_{\mathrm{L}}\left(K_{\mathrm{s}} R_{\mathrm{L}} C_{\mathrm{L}} K_{\mathrm{s}}\right) C_{\mathrm{L}}+2 R_{\mathrm{L}}\left(K_{\mathrm{s}} C_{\mathrm{L}} C_{\mathrm{L}} K_{\mathrm{s}}\right) R_{\mathrm{L}} \\
& +4 R_{\mathrm{L}} C_{\mathrm{L}} K_{\mathrm{s}}\left(R_{\mathrm{L}} K_{\mathrm{s}} C_{\mathrm{L}}\right) .
\end{aligned}
$$

The diagrams associated with Eqs. (60)-(61) are shown in Fig. 5. In Eq. (61) and in Fig. 5 we have not shown five additional terms and diagrams that vanish because they contain a two-point function $\langle\lambda \lambda\rangle_{0}$ or a closed loop over response functions $R_{\mathrm{L}}$. Indeed, the former vanishes because of Eq. (59) (and more generally Eq. (26)) whereas closed loops over $R_{\mathrm{L}}$ vanish because of the Heaviside factors $\theta\left(\eta_{1}-\eta_{2}\right)$ associated with $R_{\mathrm{L}}$ as in Eq. (19). Moreover, it happens that diagram (d) of Fig. 5 also vanishes because the right vertex $K_{\mathrm{s}}$ attached to the closed loop over $C_{\mathrm{L}}$ is of the form $K_{\mathrm{s}}(0 ; \boldsymbol{q},-\boldsymbol{q})$ (where we only write the dependence on wavenumbers), which is equal to zero from the explicit expressions (12)-(14) of the vertices $\gamma^{\mathrm{s}}$. Then, one can check that the diagram (b) gives back diagram (b) of Fig. 2 and Eq. (46) whereas diagram (c) gives back diagram (c) of Fig. 2 and Eq. (47). Thus, we indeed recover the standard perturbative results. Nevertheless, these two equivalent expansions have different diagrammatic structures. Thus, the expansion of Fig. 5, derived from the path integral (24), only involves the cubic vertex $K_{\mathrm{S}}$ but two propagators $C_{\mathrm{L}}$ and $R_{\mathrm{L}}$ (with four indices) because of the two two-component fields $\psi$ and $\lambda$. By contrast, the standard expansion of Fig. 2 involves new vertices $F_{n}^{s}$ of increasing order as we include higher-order terms but only one twopoint function: the linear density power spectrum $P_{\mathrm{L}}$. However, all kernels $F_{n}$ can be written in terms of the building blocks $R_{\mathrm{L}}$ and $K_{\mathrm{s}}$, as in Fig. 13 and Eqs. (79)-(80) below, which are also the basic blocks of the expansion in Fig. 5 . (a)

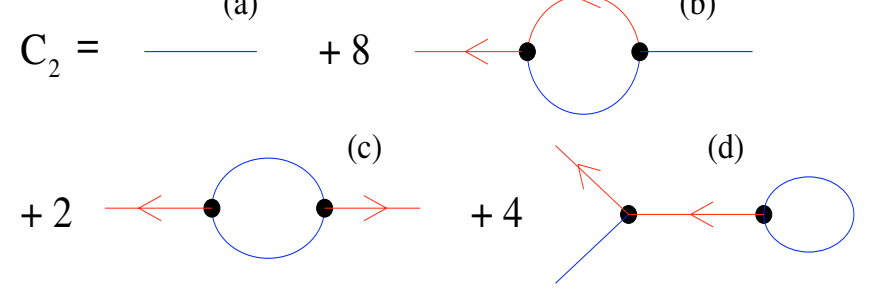

Fig. 5. The diagrams associated with the expansion (57) over powers of $K_{\mathrm{s}}$ for the two-point correlation $C_{2}$. The big dots are the three-leg vertex $K_{\mathrm{s}}$. The solid lines are the linear propagators $C_{\mathrm{L}}$ and $R_{\mathrm{L}}$ as in Fig. 4. Note that the symbols are different from those used in Figs. 13 for the standard perturbative expansions. Again we only display the diagrams obtained up to one-loop order (i.e. $P_{\mathrm{L}}^{2}$ for $C_{2}$ ).

Higher-order correlation functions can be obtained as in Eq. (57) by expanding over $K_{\mathrm{s}}$. This gives for the three-point correlation:

$C_{3}=\left\langle\psi\left(x_{1}\right) \psi\left(x_{2}\right) \psi\left(x_{2}\right)\left(\lambda K_{\mathrm{s}} \psi^{2}+\frac{\left(\lambda K_{\mathrm{s}} \psi^{2}\right)^{3}}{6}+. .\right)\right\rangle_{0}$.

Thus, we obtain up to 1-loop order

$C_{3}^{\text {tree }}=R_{\mathrm{L}} K_{\mathrm{s}} C_{\mathrm{L}} C_{\mathrm{L}}+5$ perm.,

and

$$
\begin{aligned}
C_{3}^{1 \text { loop }}= & (24+48+48)\left(K_{\mathrm{s}}^{2} R_{\mathrm{L}}^{2} C_{\mathrm{L}}^{2}\right) K_{\mathrm{s}} R_{\mathrm{L}} C_{\mathrm{L}} \\
& +24\left(K_{\mathrm{s}}^{2} R_{\mathrm{L}}^{3} C_{\mathrm{L}}\right) K_{\mathrm{s}} C_{\mathrm{L}}^{2}+8 R_{\mathrm{L}}^{3}\left(K_{\mathrm{s}}^{3} C_{\mathrm{L}}^{3}\right) \\
& +48 R_{\mathrm{L}}^{2} C_{\mathrm{L}}\left(K_{\mathrm{s}}^{3} R_{\mathrm{L}} C_{\mathrm{L}}^{2}\right)+(24+48) R_{\mathrm{L}} C_{\mathrm{L}}^{2}\left(K_{\mathrm{s}}^{3} R_{\mathrm{L}}^{2} C_{\mathrm{L}}\right)
\end{aligned}
$$

We show the diagrams associated with Eqs. (63)-(64) in Fig. 6. We can note that the first four one-loop diagrams, (b), (c), (d), and (e), are 1-particle reducible: they can be disconnected by cutting the two-point function which connects the "bubble" to the rightmost vertex $K_{\mathrm{s}}$. The comparison with Fig. 5 shows that they correspond to the perturbative corrections of the two-point functions. Thus, the first three one-loop diagrams can be obtained from the tree diagram (a) by inserting the one-loop corrections (b) and (c) of Fig. 5 into the two-point correlations $C_{\mathrm{L}}$ whereas the fourth one can be obtained by inserting the one-loop correction of the response $R$. The last four one-loop diagrams (f), (g), (h), and (i), of Fig. 6, correspond to perturbative corrections to the vertex $K_{\mathrm{s}}$ itself. Note that whereas the last two diagrams respect the structure of the bare vertex $K_{\mathrm{s}}$ (attached to one response field $\lambda$ and two fields $\psi$ ) the other two diagrams bring up vertices attached to three fields $\lambda$ or to two fields $\lambda$ and one field $\psi$. Thus all possible configurations are generated beyond tree-level for the "renormalized" vertex. As for the two-point function diagrams shown in Fig. 5, we have only displayed in Fig. 6 the non-vanishing diagrams. For instance, the renormalized vertex which would arise from a closed triangular loop over three responses $R_{\mathrm{L}}$ vanishes because of the Heaviside factors associated with the response $R_{\mathrm{L}}$ (that express causality).

Again, one can check that Eqs. (63), (64) give back the results obtained from standard perturbation theory in Eq. (48) and Fig. 3. Thus, from the expression (11) of the bare vertex $K_{\mathrm{s}}$, the tree-order diagram (63) reads as

$$
\begin{aligned}
& C_{3}^{\mathrm{tree}}\left(x_{1}, x_{2}, x_{3}\right)=\delta_{\mathrm{D}}\left(\boldsymbol{k}_{1}+\boldsymbol{k}_{2}+\boldsymbol{k}_{3}\right) \int_{-\infty}^{\eta_{1}} \mathrm{~d} \eta_{1}^{\prime} R_{L i_{1} i_{1}^{\prime}}\left(k_{1} ; \eta_{1}, \eta_{1}^{\prime}\right) \\
& \quad \times C_{L i_{2} i_{2}^{\prime}}\left(k_{2} ; \eta_{2}, \eta_{1}^{\prime}\right) C_{L i_{3} i_{3}}\left(k_{3} ; \eta_{3}, \eta_{1}^{\prime}\right) \gamma_{i_{1}^{\prime} ; i_{2}^{\prime}, i_{3}^{\prime}}^{\mathrm{s}}\left(\boldsymbol{k}_{2}, \boldsymbol{k}_{3}\right)+5 \text { perm. }(65)
\end{aligned}
$$




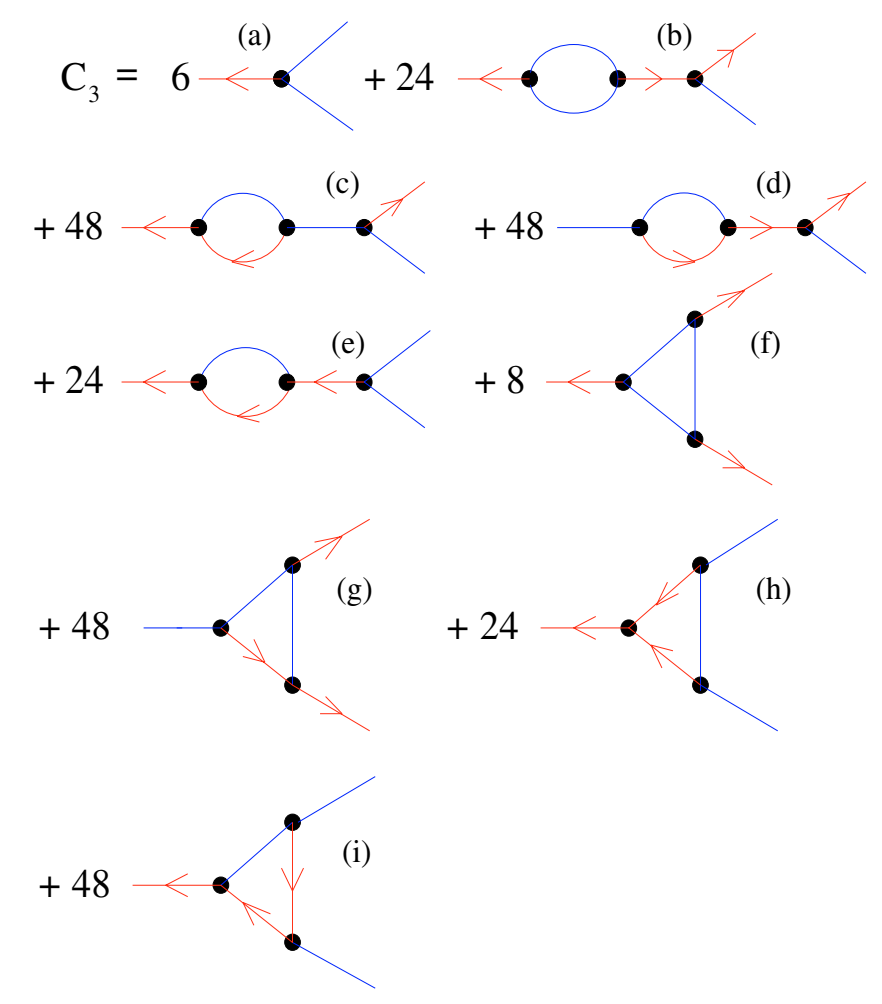

Fig. 6. The diagrams associated with the expansion (62) over powers of $K_{\mathrm{s}}$ for the three-point correlation $C_{3}$. The symbols are as in Fig. 5. Again we only display the diagrams obtained up to one-loop order (i.e. $P_{\mathrm{L}}^{3}$ for $\left.C_{3}\right)$.

Substituting the expressions of the linear two-point functions in the right hand side of Eq. (65) and performing the integral over the time $\eta_{1}^{\prime}$ we recover Eq. (51) for $i_{1}=i_{2}=i_{3}=1$.

Higher-order correlations $C_{\mathrm{p}}$ are computed in the same fashion, by inserting the factor $\left(\psi_{1} . . \psi_{p}\right)$ in front of the exponential in Eq. (24) and expanding over the cubic part of the action. The same technique also provides higher-order response functions, such as

$$
\begin{aligned}
R_{\psi \psi \lambda} & \equiv\left\langle\psi\left(x_{1}\right) \psi\left(x_{2}\right) \lambda\left(x_{3}\right)\right\rangle=\left.\frac{\delta}{\delta \zeta\left(x_{3}\right)}\right|_{\zeta=0}\left\langle\psi\left(x_{1}\right) \psi\left(x_{2}\right)\right\rangle \\
& =\left.\frac{\delta C\left(x_{1}, x_{2}\right)}{\delta \zeta\left(x_{3}\right)}\right|_{\zeta=0} .
\end{aligned}
$$

Thus, $R_{\psi \psi \lambda}$ measures the response of the two-point correlation $C$ to an infinitesimal external perturbation $\zeta$ added to the right hand side of the equation of motion (8). It is also related to the crosscorrelation of the nonlinear field $\psi$ with the initial condition $\psi_{I}$ at a finite time $\eta_{\mathrm{I}}$ as

$$
\left\langle\psi\left(x_{1}\right) \psi\left(x_{2}\right) \psi_{I}\left(\bar{x}_{3}\right)\right\rangle=R_{\psi \psi \lambda}\left(x_{1}, x_{2} ; \bar{x}, \eta_{\mathrm{I}}\right) \times G_{I}\left(\bar{x}, \bar{x}_{3}\right),
$$

where the product does not involve any integration over time and we note $\bar{x}=(\boldsymbol{k}, i)$, see Eq. (49) of Valageas (2007b) for details in the case of the two-point response function.

Note that throughout this article we take the limit $\eta_{\mathrm{I}} \rightarrow-\infty$ (i.e. $D_{I} \rightarrow 0$ ). This applies both to the standard perturbation theory of Sect. 3 (and the expansion (79) below), which allows us to write the solution of the equations of motion as an expansion over the linear growing mode, and to the methods derived from the path integral (24). It is possible to apply the path-integral for-

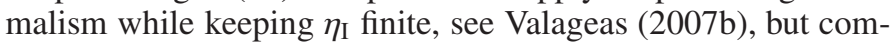
putations simplify in the limit $\eta_{\mathrm{I}} \rightarrow-\infty$. In a similar fashion, keeping $\eta_{\mathrm{I}}$ finite would add decaying terms to the standard expansion (40).

\section{Large- $N$ methods}

We now describe in this section how to obtain the threepoint density and velocity correlations from the large- $N$ methods introduced in Sect. 2.3. From the path-integral formalism (24), (25) the three-point correlation $C_{3}$ can be written as (Zinn-Justin 1989; Valageas 2007a)

$C_{3}\left(x_{1}, x_{2}, x_{3}\right)=-C\left(x_{1}, x_{1}^{\prime}\right) C\left(x_{2}, x_{2}^{\prime}\right) C\left(x_{3}, x_{3}^{\prime}\right) \Gamma_{3}\left(x_{1}^{\prime}, x_{2}^{\prime}, x_{3}^{\prime}\right)$

where $\Gamma_{3}$ is the symmetric dressed three-point vertex (the socalled three-point proper vertex of the 1PI effective action $\Gamma[\psi]$ ). Here we put on the same footing the fields $\psi$ and $\lambda$ so that twopoint functions $C$ correspond to all three possibilities $\langle\psi \psi\rangle=$ $C,\langle\psi \lambda\rangle=R$, and $\langle\lambda \lambda\rangle=0$, and similarly for the three-point function $C_{3}$. Then, the proper vertex $\Gamma_{3}$ can be obtained from a series of three-leg diagrams, up to the required order over $1 / N$.

In this article we restrict ourselves to the three-point correlation $C_{3}\left(x_{1}, x_{2}, x_{3}\right)$ defined as

$C_{3}\left(x_{1}, x_{2}, x_{3}\right)=\left\langle\psi\left(x_{1}\right) \psi\left(x_{2}\right) \psi\left(x_{3}\right)\right\rangle$.

It describes both density and velocity correlations, as well as their cross-correlations. We do not study in this paper threepoint functions such as $\langle\psi \psi \lambda\rangle$ of Eq. (66), associated with the functional derivative of the two-point correlation with respect to an external noise (and in particular with respect to the initial conditions). Of course, higher-order correlation and response functions can be obtained in a similar manner, from the twopoint function $C$ and $R$ and the higher-order proper vertices $\Gamma_{\mathrm{p}}$. However, since for practical purposes higher-order statistics are increasingly noisy we do not go beyond three-point functions in this paper.

Note that the structure of the large- $N$ expansions is somewhat different from the expansions described in Sects. 3, 4. Indeed, contrary to the latter cases, the contribution associated with a given diagram is not fixed but depends on the order up to which the expansion is performed. This arises from the fact that each diagram depends on the nonlinear two-point functions $R$ and $C$ (more precisely the approximated nonlinear twopoint functions computed at this order). Thus, the diagrammatic series actually gives an implicit equation for $R$ and $C$, as in Eqs. (27), (28) where the right hand side depends on $R$ and $C$.

Then, the order of these expansions is set by the order of the diagrams kept in the self-energies $\Sigma$ and $\Pi$. At tree-order, we merely have $\Sigma=0$ and $\Pi=0$, at one-loop order we have either Eqs. (29), (30) or Eqs. (31), (32), and at higher orders we would need to include higher-order diagrams (that would no longer look identical for the steepest-descent and 2PI methods).

\subsection{Direct steepest-descent method}

We first consider in this section the direct steepest-descent method of Sect. 2.4. At tree-order, the two-point functions are equal to the linear two-point functions (Valageas 2004, 2007a) whereas the proper vertex $\Gamma_{3}$ is equal to the bare vertex of the action $S[\psi, \lambda]$ which yields from Eq. (25)

$\Gamma_{3}^{\text {tree }}\left(x_{1}, x_{2}, x_{3}\right)=-K_{\mathrm{s}}\left(x_{1} ; x_{2}, x_{3}\right)+5$ perm.,

with a multiplicity factor $6=3$ ! which corresponds to all permutations of the triplet $\left(x_{1}, x_{2}, x_{3}\right)$. Therefore, at tree-order we 


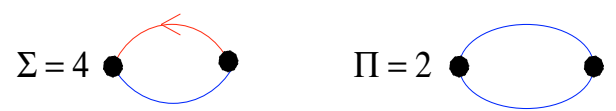

Fig. 7. The one-loop diagrams for the self-energies $\Sigma$ and $\Pi$ obtained from the direct steepest-descent expansion.

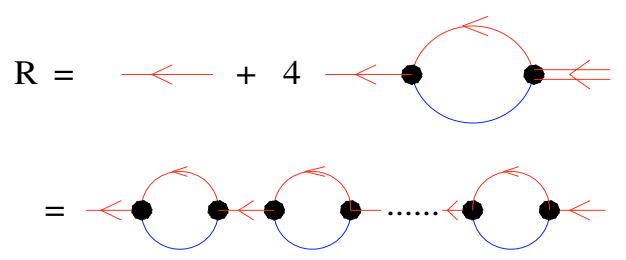

Fig. 8. The diagrams obtained at one-loop order for the response $R$ from the direct steepest-descent expansion. The first equality corresponds to the implicit Eq. (71), where we note by double lines the nonlinear response $R$. The second equality shows the infinite series of bubble diagrams resummed by this large- $N$ method, as in Eq. (72).

recover exactly the tree-diagrams (a) of Figs. 5, 6, obtained by expanding over the nonlinear coupling $K_{\mathrm{s}}$, hence the lowestorder results of standard perturbation theory.

At one-loop order, the two-point functions are obtained from the system (27), (28) together with Eqs. (29), (30) for the selfenergy. The diagrammatic expression of the self-energy is shown at one-loop order in Fig. 7. Then, the solution of Eq. (28) can be written as

$$
\begin{aligned}
R & =R_{\mathrm{L}}+R_{\mathrm{L}} \cdot \Sigma \cdot R \\
& =R_{\mathrm{L}}+R_{\mathrm{L}} \cdot \Sigma \cdot R_{\mathrm{L}}+R_{\mathrm{L}} \cdot \Sigma \cdot R_{\mathrm{L}} \cdot \Sigma \cdot R_{\mathrm{L}}+\ldots
\end{aligned}
$$

In the first line we used the second Eq. (23) to obtain the integral form of Eq. (28) and in the second line we wrote the solution as a series over powers of $\Sigma$. Equation (72) explicitly shows that at "one-loop order" the steepest-descent method has performed the resummation of the infinite series of bubble diagrams displayed in Fig. 8.

Let us stress here that for the large- $N$ methods, the loop order of the approximation refers to the truncation order of the infinite series of diagrams obtained for the self-energies, and not to the truncation order of the correlation and response functions. Indeed, any finite order in terms of the self-energy diagrams automatically leads to an infinite partial resummation over all orders for the diagrams associated with the correlation and response functions. However, these resummations are only complete up to the same order as for the self-energy (we miss some of the higher-order diagrams). Thus, at one-loop order, taking into account the only two diagrams of Fig. 7 for the self-energies actually gives rise to the infinite series shown in Figs. 8, 9 for the physical two-point functions $R$ and $C$, which include contributions that contain an arbitrary number of loops. However, these partial resummations are complete only up to one-loop, that is they miss some two-loop and higher-order diagrams, such as the one of Fig. 14 for $C$.

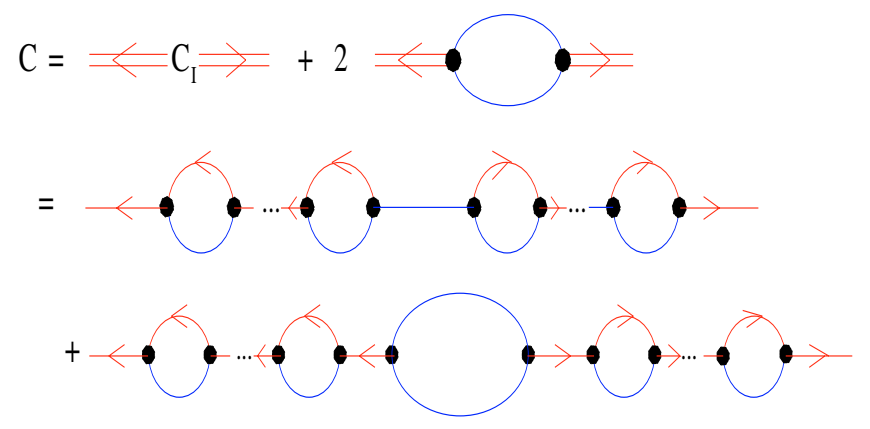

Fig. 9. The diagrams obtained at one-loop order for the two-point correlation $C$ from the direct steepest-descent expansion. The first equality corresponds to Eq. (73), where we note by double lines the nonlinear response $R$. The second equality shows the two infinite series of bubble diagrams resummed by this large- $N$ method, in terms of the linear functions $C_{\mathrm{L}}$ and $R_{\mathrm{L}}$.

Next, the solution of Eq. (27) can be written as (Valageas 2004, 2007a)

$C=R \times C_{\mathrm{L}}\left(\eta_{\mathrm{I}}\right) \times R^{\mathrm{T}}+R \cdot \Pi \cdot R^{\mathrm{T}}$,

where the first product does not contain any integration over time,

$$
\begin{aligned}
& R \times C_{\mathrm{L}}\left(\eta_{\mathrm{I}}\right) \times R^{\mathrm{T}}=\delta_{\mathrm{D}}\left(\boldsymbol{k}_{1}+\boldsymbol{k}_{2}\right) \sum_{i_{1}^{\prime} i_{2}^{\prime}} R_{i_{1} i_{1}^{\prime}}\left(k_{1} ; \eta_{1}, \eta_{\mathrm{I}}\right) \\
& \quad \times C_{L i_{1}^{\prime} i_{2}^{\prime}}\left(k_{1} ; \eta_{\mathrm{I}}, \eta_{\mathrm{I}}\right) R_{i_{2} i_{2}^{\prime}}\left(k_{1} ; \eta_{2}, \eta_{\mathrm{I}}\right),
\end{aligned}
$$

and we let the initial time go to the infinite past $\eta_{\mathrm{I}} \rightarrow-\infty$. Moreover, we can note that in the linear regime we have

$C_{\mathrm{L}}=R_{\mathrm{L}} \times C_{\mathrm{L}}\left(\eta_{\mathrm{I}}\right) \times R_{\mathrm{L}}^{\mathrm{T}}$

as can be checked from the explicit expressions of $C_{\mathrm{L}}$ and $R_{\mathrm{L}}$. From Eqs. (72)-(75) and Fig. 8 we see that the infinite series of diagrams resummed by the steepest-descent method at one-loop order are the two series of bubble diagrams shown in Fig. 9. We can check that we recover the nonzero diagrams obtained at oneloop order in Fig. 5 within the perturbative expansion over the cubic interaction $K_{\mathrm{s}}$. In addition, the implicit Eqs. (27), (28) have allowed us to resum two infinite series of higher-order diagrams. Thus, we recover the fact that the large- $N$ expansions and the standard perturbative expansions agree up to the truncation order (here one-loop) and only differ by higher-order terms (Valageas 2004, 2007a).

Note that we do not recover the diagram (d) of Fig. 5 that was equal to zero. In fact, the property $K_{\mathrm{s}}(0 ; \boldsymbol{q},-\boldsymbol{q})=0$ that sets this diagram to zero has already been used twice to derive the Schwinger-Dyson Eqs. (27)-(30): i) to obtain the path integral (24) where we used that the Jacobian is an irrelevant constant, and ii) to obtain Eqs. (27)-(30) by expanding over $1 / N$ where we used that $\langle\psi\rangle=0$ (which also comes from $\left.K_{\mathrm{S}}(0 ; \boldsymbol{q},-\boldsymbol{q})=0\right)$. Then, the explicit use of these simplifications has already removed from the large- $N$ expansion diagrams such as the diagram (d) of Fig. 5.

For the three-point correlation $C_{3}$ we need the one-loop contribution to the three-point vertex $\Gamma_{3}$ which reads as (e.g. Zinn-Justin 1989; Valageas 2004)

$\Gamma_{3}^{1 \text { loop }}=-6^{3} K_{\mathrm{s}} C_{0} K_{\mathrm{s}} C_{0} K_{\mathrm{s}} C_{0}$,

where the two-point functions $C_{0}$ form a loop which joins the three bare vertices $K_{\mathrm{s}}$. Here $C_{0}$ is the auxiliary two-point function introduced within the direct steepest-descent scheme as in 


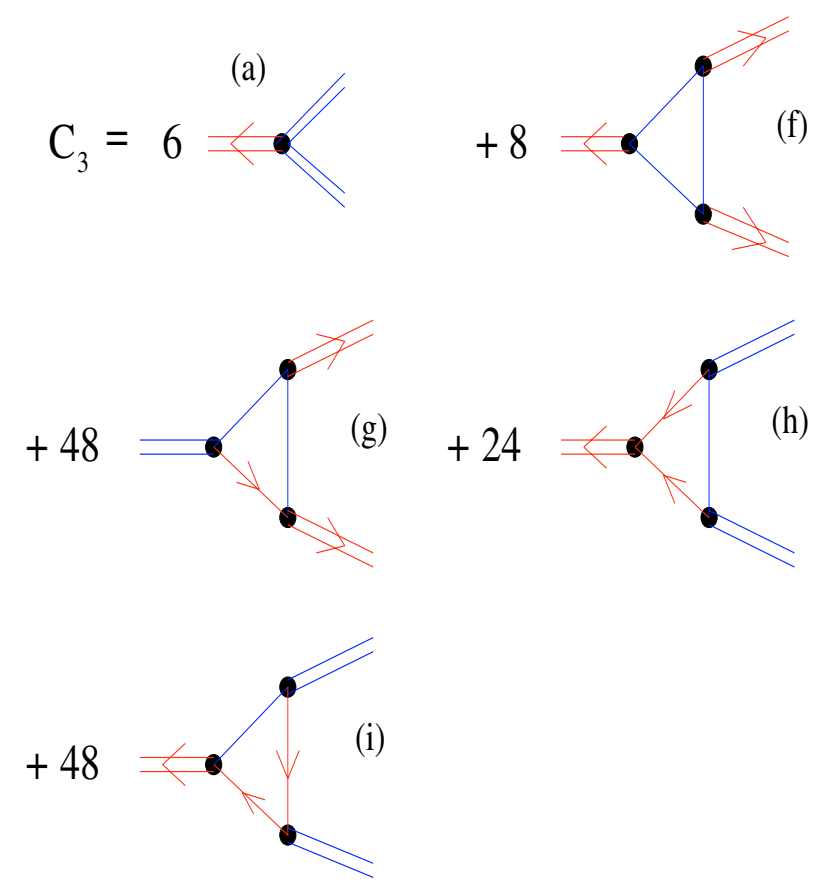

Fig. 10. The diagrams obtained at one-loop order for the three-point correlation $C_{3}$ from the direct steepest-descent expansion. The double lines are again the nonlinear response $R$ and the nonlinear correlation $C$. We use the same labels as in Fig. 6. The four diagrams (b) to (e) of Fig. 6 do not appear in this expansion scheme since their contribution is included in diagram (a) through the use of nonlinear two-point functions instead of linear ones in the external legs.

Sect. 2.4, which happens to be equal to the linear two-point function $C_{\mathrm{L}}$. Then, from Eq. (68) the "tree-diagram" contribution reads as (using Eq. (70))

$C_{3}^{\text {tree }}=6 C C C . K_{\mathrm{s}}$,

whereas the "one-loop diagram" reads as (using Eq. (76))

$C_{3}^{1 \text { loop }}=216 C C C .\left(K_{\mathrm{s}} C_{\mathrm{L}} K_{\mathrm{s}} C_{\mathrm{L}} K_{\mathrm{s}} C_{\mathrm{L}}\right)$.

We show in Fig. 10 the diagrams associated with Eqs. (77), (78) that give the three-point correlation at one-loop order within the steepest-descent expansion. In both Eqs. (77), (78) we must use the nonlinear two-point functions $C$ computed at the same order within the steepest-descent method (here one-loop). That is, from the one-loop diagrams of Fig. 7 for the self-energy, which lead to the infinite loop resummations for two-point functions shown in Figs. 8, 9. (Let us recall that the "loop-order" refers to the diagrams kept for the self-energy, but the two-point functions $R$ and $C$ contain resummations over diagrams that contain an arbitrary number of loops over the linear propagators.)

The three nonlinear two-point functions $C$ that appear in Eqs. (77), (78) are shown in Fig. 10 by the double lines associated with the three external legs, as in Eq. (68). Thus, the contribution (77), shown by diagram (a) in Fig. 10, is no longer equal to the standard tree-diagram (a) of Fig. 6 associated with Eqs. (63) and (65). It includes three infinite series of additional diagrams, such as the series shown in Fig. 11, which are obtained by replacing the nonlinear two-point functions $R$ and $C$ by their diagrammatic expressions shown in Figs. 8, 9. In particular, these series contain the first four one-loop diagrams (b), (c), (d), and (e), of the expansion over $K_{\mathrm{s}}$ shown in Fig. 6. The other diagrams of Fig. 10 also contain such infinite series once written in terms of the linear two-point functions $R_{\mathrm{L}}$ and $C_{\mathrm{L}}$.

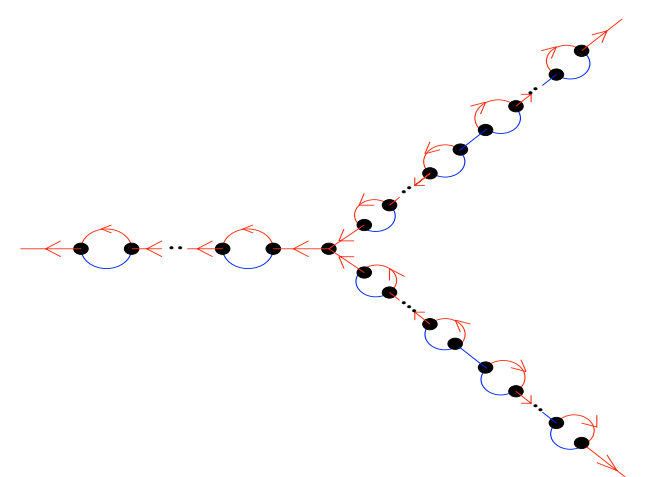

Fig. 11. An infinite series of diagrams resummed by the "tree-diagram" of Fig. 10 of the steepest-descent expansion at one-loop order. It is obtained from the diagrammatic expansions of the nonlinear $R$ and $C$ shown in Figs. 8, 9. There are two more series (not shown) obtained by taking into account the second diagram of Fig. 9.

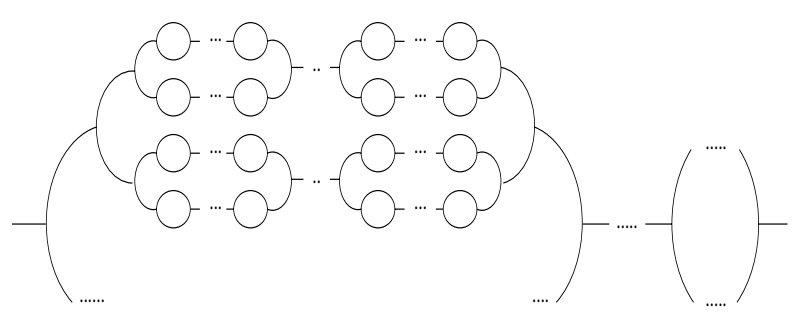

Fig. 12. An infinite series of diagrams included in the two-point functions given by the 2PI effective action expansion at one-loop order. There are additional infinite series of diagrams as compared with the steepest-descent method thanks to the "renormalization" of the selfenergy terms.

Thus, the steepest-descent method has allowed us to resum in an automatic manner some partial infinite series of diagrams for all many-body correlation functions.

\section{2. $2 P I$ effective action method}

As recalled in Sect. 2.5, the 2PI effective action method also gives the Schwinger-Dyson Eqs. (27), (28). In particular, Eqs. (71)-(73) still apply but they are now nonlinear as the selfenergy terms now depend on the nonlinear two-point functions as in Eqs. (31), (32). At tree-order we again recover the results of the standard perturbative expansion. At one loop-order the self-energy is given by the same diagrams as those of Fig. 7 except that the internal two-point functions are the nonlinear ones (i.e. single lines must be replaced by double lines). Then, it is clear that the two-point functions obtained from Eqs. (72), (73) contain the same infinite series of diagrams as those derived in the steepest-descent method, shown in Figs. 8, 9, but also additional series due to the "renormalization" of the self-energy terms. For illustration we display in Fig. 12 a typical diagram included in the 2PI effective action resummation for the two-point functions $R$ and $C$.

Next, the three-point proper vertex $\Gamma_{3}$ at one loop-order can also be written as Eq. (76), where the linear two-point functions $C_{0}$ must be replaced by the nonlinear ones $C$ (i.e. all single lines including the internal ones must be replaced by double lines). Expanding back over the linear two-point functions $R_{\mathrm{L}}$ and $C_{\mathrm{L}}$ we recover again all diagrams obtained within the steepest-descent method as well as additional series which include terms such as those associated with Fig. 12. 

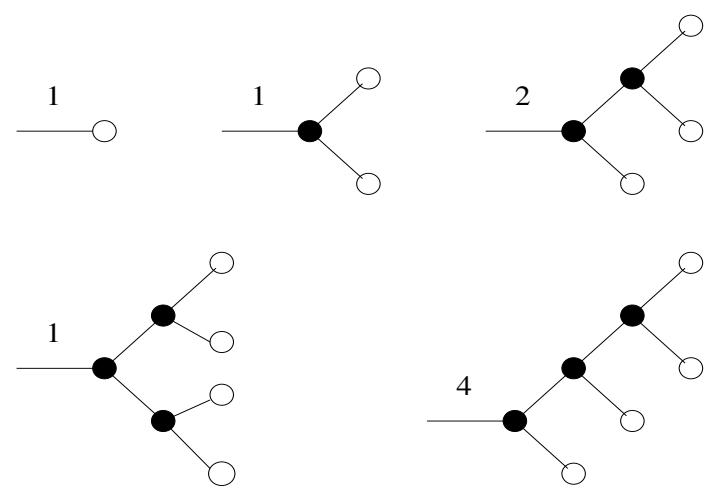

Fig. 13. The expansion of the nonlinear field $\psi$ over the linear growing mode $\psi_{\mathrm{L}}$ from Eq. (79), up to order $\psi_{\mathrm{L}}^{4}$. The filled circles are the vertex $\tilde{K}_{\mathrm{s}}$ of Eq. (80), whereas the white circles are the linear growing mode $\psi_{\mathrm{L}}$. The numbers are the multiplicity factor associated with each diagram.

\section{Random advection in the large-k limit}

As recalled in Sect. 3, the standard perturbation theory associated with Eq. (40) writes the nonlinear density field $\delta(\boldsymbol{k})$ as a series over powers of the linear density contrast $\delta_{\mathrm{L}}$. The kernels $F_{n}$ of Eq. (40) are computed by recursion from the equation of motion (8) so that each order involves a new vertex $F_{n}$. However, it is clear that the latter can also be written in terms of the vertex $K_{\mathrm{s}}$ that appears in Eq. (8). Thus, the equation of motion (8) for the two-component field $\psi$ can be solved through the expansion over the linear growing mode $\psi_{\mathrm{L}}$ of Eq. (16) as (Crocce \& Scoccimarro 2006a,b; Valageas 2007b)

$\psi=\psi_{\mathrm{L}}+\tilde{K}_{\mathrm{s}} \psi_{\mathrm{L}}^{2}+2 \tilde{K}_{\mathrm{s}}^{2} \psi_{\mathrm{L}}^{3}+5 \tilde{K}_{\mathrm{s}}^{3} \psi_{\mathrm{L}}^{4}+\ldots$,

where we introduced the integral vertex $\tilde{K}_{\mathrm{s}}$ defined from $K_{\mathrm{s}}$ and the linear response $R_{\mathrm{L}}$ by

$\tilde{K}_{\mathrm{s}}=R_{\mathrm{L}} \cdot K_{\mathrm{s}}$.

The diagrams associated with the series (79) are shown in Fig. 13, see Crocce \& Scoccimarro (2006a). Of course, Eq. (79) and Fig. 13 are equivalent to the standard perturbation series of Eq. (40) and Fig. 1. Then, with the aim of computing the response function $R$, Crocce \& Scoccimarro (2006a) noticed that it is possible to perform a partial resummation of the diagrams shown in Fig. 13 in a high- $k$ limit. First, one only keeps the diagrams such as the last one in Fig. 13, where one can define a "principal path" connecting a linear mode $\psi_{\mathrm{L}}$ to the left root so that all other linear modes $\psi_{\mathrm{L}}$ are directly connected to this path. (This is not the case for the other diagram of order $\psi_{\mathrm{L}}^{4}$ in Fig. 13.) Second, in a high- $k$ limit one may approximate the vertex $K_{\mathrm{s}}$ by

$$
\begin{aligned}
k \gg k_{1}: K_{\mathrm{s}}\left(x ; x_{1}, x_{2}\right) \simeq & \delta_{\mathrm{D}}\left(\boldsymbol{k}_{2}-\boldsymbol{k}\right) \delta_{\mathrm{D}}\left(\eta_{1}-\eta\right) \delta_{\mathrm{D}}\left(\eta_{2}-\eta\right) \\
& \times \frac{\boldsymbol{k} \cdot \boldsymbol{k}_{1}}{2 k_{1}^{2}} \delta_{i, i_{2}} \delta_{i_{1}, 2} .
\end{aligned}
$$

This simplifies the series (79) which can be resummed to give the response function (Crocce $\&$ Scoccimarro 2006a,b)

$R\left(x_{1}, x_{2}\right)=R_{\mathrm{L}}\left(x_{1}, x_{2}\right) \mathrm{e}^{-\left(D_{1}-D_{2}\right)^{2} k^{2} \sigma_{v}^{2} / 2}$,

where $\sigma_{v}^{2}$ is the variance of the one-dimensional linear displacement field $s_{\mathrm{L} 0}$ (equal to the variance of the one-dimensional linear velocity dispersion up to a normalization factor) given by

$\sigma_{v}^{2}=\frac{4 \pi}{3} \int_{0}^{\infty} \mathrm{d} k P_{\mathrm{L} 0}(k)$

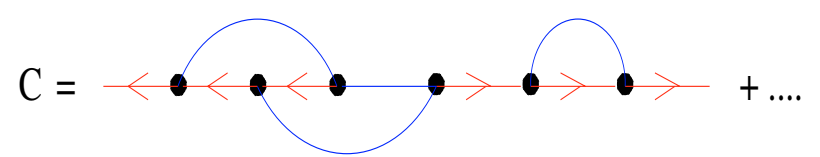

Fig. 14. A diagram contained in the high- $k$ resummation associated with Eq. (84) for the two-point correlation $C$.

As seen in Valageas (2007b), keeping only the "principal path" diagrams amounts to approximate the equation of motion (8) by the linearized equation

$O . \psi=2 K_{\mathrm{s}} \psi_{\mathrm{L}} \psi, \quad$ or $\quad \psi=\psi_{\mathrm{L}}+2 \tilde{K}_{\mathrm{s}} \psi_{\mathrm{L}} \psi$.

Then, using the approximation (81), the solution of Eq. (84) reads as (Valageas 2007b)

$\delta(\boldsymbol{k})=\delta_{\mathrm{L}}(\boldsymbol{k}) \mathrm{e}^{\int \mathrm{d} \boldsymbol{q} \frac{\boldsymbol{k} \cdot \boldsymbol{q}}{q^{2}} \delta_{\mathrm{L}}(\boldsymbol{q})}$.

The key simplification comes from the approximation $\delta_{\mathrm{D}}\left(\boldsymbol{k}_{1}+\right.$ $\left.\boldsymbol{k}_{2}-\boldsymbol{k}\right) \simeq \delta_{\mathrm{D}}\left(\boldsymbol{k}_{2}-\boldsymbol{k}\right)$ in Eq. (81). This makes all the intermediate legs in the simplified diagrammatic series factorize as powers of $\int \mathrm{d} \boldsymbol{q} \frac{k \cdot \boldsymbol{q}}{q^{2}} \delta_{\mathrm{L}}\left(\boldsymbol{q}, \eta^{\prime}\right)$ which allows the resummation (85). Then, it is easy to derive Eq. (82) from Eq. (85), as well as

$C\left(x_{1}, x_{2}\right)=C_{\mathrm{L}}\left(x_{1}, x_{2}\right) \mathrm{e}^{-\left(D_{1}-D_{2}\right)^{2} k^{2} \sigma_{v}^{2} / 2}$.

As discussed in Valageas (2007b), this procedure has actually replaced the gravitational dynamics by the simple effective dynamics

$\delta(\boldsymbol{x}, D)=\delta_{\mathrm{L}}\left(\boldsymbol{x}-\boldsymbol{s}_{L}\left(\boldsymbol{x}_{\mathrm{Lag}}=0, D\right), D\right)$.

That is, the linear density field is uniformly advected by the linear displacement $\boldsymbol{s}_{L}\left(\boldsymbol{x}_{\mathrm{Lag}}=0, D\right)$ associated with the Lagrangian position $\boldsymbol{x}_{\mathrm{Lag}}=0$. Then, the average over this random Gaussian displacement leads to the apparent loss of memory seen in Eqs. (82), (86), through the Gaussian decay $\mathrm{e}^{-D^{2} k^{2} \sigma_{v}^{2} / 2}$. As discussed in Valageas (2007b), it happens that for the Zeldovich dynamics Eq. (82) actually gives the exact nonlinear response whereas Eq. (86) gives the correct Gaussian decay for different times $D_{1} \neq D_{2}$ but does not capture the equal-time behavior of the nonlinear correlation $C$.

As compared with the large- $N$ resummation of Sect. 5.1, which gives the bubble diagrams of Fig. 9 for the two-point correlation $C$, the resummation associated with Eq. (84) (i.e. keeping the principal path diagrams for $\psi$ ) contains additional diagrams such as the one shown in Fig. 14. Indeed, when we join the relevant diagrams of Fig. 13 to compute $C\left(x_{1}, x_{2}\right)=$ $\left\langle\psi\left(x_{1}\right) \psi\left(x_{2}\right)\right\rangle$, the Gaussian average draws all possible pairs from all secondary legs $\psi_{\mathrm{L}}\left(\boldsymbol{q}_{j}\right)$ that connect to the principal path (including those obtained by matching fields $\psi_{\mathrm{L}}\left(\boldsymbol{q}_{j}\right)$ that are associated with both nonlinear fields $\psi\left(x_{1}\right)$ and $\left.\psi\left(x_{2}\right)\right)$. By contrast, the bubble diagrams of Fig. 9 only involve the Gaussian pairings performed in sequential order as we move along the principal path (but this method does not require the high- $k$ approximation (81)).

Nevertheless, at one-loop order we can substitute the twopoint functions (82), (86), obtained in this fashion into the diagrams of Fig. 10 to derive an approximation for the three-point function that is correct up to this order and contains infinite partial resummations. We can obtain two approximations for $C_{3}$ in this manner. We can either use Eqs. (82), (86), into the external lines only of the one-loop diagrams of Fig. 10, in the spirit of the steepest-descent scheme of Sect. 5.1, or into both the external and internal lines in the spirit of the 2PI effective action method of Sect. 5.2. As for previous schemes, these two procedures may be applied to all higher-order correlation and response functions. 


\section{Zeldovich dynamics}

We investigate in this section the predictions of the various expansion schemes described in the previous sections for the simpler case of the Zeldovich dynamics (Zeldovich 1970), where the trajectories of particles follow the linear displacement field as in Eq. (35). We focus on the three-point function $C_{3}$, and more specifically on the matter density bispectrum defined in Eq. (50), since the two-point functions $C$ and $R$ have already been studied in Valageas (2007b).

\subsection{Standard perturbation theory}

As is well-known, from the exact solution (35) of the Zeldovich dynamics the kernels $F_{n}$ introduced in the standard perturbative expansion (40) can be directly derived at any order (Grinstein \& Wise 1987). For instance, expanding the exponential in Eq. (38) gives

$\delta(\boldsymbol{k})=\sum_{n=1}^{\infty} \frac{1}{n !} \int \frac{\mathrm{d} \boldsymbol{q}}{(2 \pi)^{3}} \mathrm{e}^{-\mathrm{i} \boldsymbol{k} \cdot \boldsymbol{q}}\left(-\mathrm{i} \boldsymbol{k} \cdot \boldsymbol{s}_{L}\right)^{n}$.

Using Eq. (39) we recover Eq. (40) with the symmetric kernels

$F_{n}^{s}\left(\boldsymbol{q}_{1}, \ldots, \boldsymbol{q}_{n}\right)=\frac{1}{n !} \frac{\boldsymbol{k} \cdot \boldsymbol{q}_{1}}{q_{1}^{2}} \ldots \frac{\boldsymbol{k} \cdot \boldsymbol{q}_{n}}{q_{n}^{2}}$.

Then, using Eqs. (45)-(47) the power spectrum reads up to oneloop order as

$$
\begin{gathered}
P(k)=P_{\mathrm{L}}(k)-P_{\mathrm{L}}(k) \int \mathrm{d} \boldsymbol{q} \frac{(\boldsymbol{k} \cdot \boldsymbol{q})^{2}}{q^{4}} P_{\mathrm{L}}(q)+\int \mathrm{d} \boldsymbol{q}_{1} \mathrm{~d} \boldsymbol{q}_{2} \\
\times \delta_{\mathrm{D}}\left(\boldsymbol{q}_{1}+\boldsymbol{q}_{2}-\boldsymbol{k}\right) P_{\mathrm{L}}\left(q_{1}\right) P_{\mathrm{L}}\left(q_{2}\right) \frac{\left(\boldsymbol{k} \cdot \boldsymbol{q}_{1}\right)^{2}\left(\boldsymbol{k} \cdot \boldsymbol{q}_{2}\right)^{2}}{2 q_{1}^{4} q_{2}^{4}} .
\end{gathered}
$$

Eqs. (51)-(56) read for the bispectrum up to one-loop order as

$$
B^{\text {tree }}\left(k_{1}, k_{2}, k_{3}\right)=P_{\mathrm{L}}\left(k_{2}\right) P_{\mathrm{L}}\left(k_{3}\right) \frac{\left(\boldsymbol{k}_{1} \cdot \boldsymbol{k}_{2}\right)\left(\boldsymbol{k}_{1} \cdot \boldsymbol{k}_{3}\right)}{k_{2}^{2} k_{3}^{2}}+2 \text { perm. }
$$

and,

$$
\begin{aligned}
B^{(b)}= & -P_{\mathrm{L}}\left(k_{2}\right) P_{\mathrm{L}}\left(k_{3}\right) \frac{\left(\boldsymbol{k}_{1} \cdot \boldsymbol{k}_{2}\right)\left(\boldsymbol{k}_{1} \cdot \boldsymbol{k}_{3}\right)}{2 k_{2}^{2} k_{3}^{2}} \int \mathrm{d} \boldsymbol{q} P_{\mathrm{L}}(q) \frac{\left(\boldsymbol{k}_{1} \cdot \boldsymbol{q}\right)^{2}}{q^{4}} \\
& +2 \text { perm., }
\end{aligned}
$$

$$
\begin{aligned}
B^{(c)}= & -P_{\mathrm{L}}\left(k_{2}\right) P_{\mathrm{L}}\left(k_{3}\right) \frac{\left(\boldsymbol{k}_{1} \cdot \boldsymbol{k}_{2}\right)\left(\boldsymbol{k}_{1} \cdot \boldsymbol{k}_{3}\right)}{2 k_{2}^{2} k_{3}^{2}} \int \mathrm{d} \boldsymbol{q} P_{\mathrm{L}}(q) \frac{\left(\boldsymbol{k}_{2} \cdot \boldsymbol{q}\right)^{2}}{q^{4}} \\
& +5 \text { perm., }
\end{aligned}
$$

$$
\begin{aligned}
& B^{(d)}=-P_{\mathrm{L}}\left(k_{1}\right) \frac{\boldsymbol{k}_{1} \cdot \boldsymbol{k}_{3}}{2 k_{1}^{2}} \int \mathrm{d} \boldsymbol{q}_{1} \mathrm{~d} \boldsymbol{q}_{2} \delta_{\mathrm{D}}\left(\boldsymbol{q}_{1}+\boldsymbol{q}_{2}-\boldsymbol{k}_{2}\right) \\
& \quad \times P_{\mathrm{L}}\left(q_{1}\right) P_{\mathrm{L}}\left(q_{2}\right) \frac{\left(\boldsymbol{k}_{2} \cdot \boldsymbol{q}_{1}\right)\left(\boldsymbol{k}_{2} \cdot \boldsymbol{q}_{2}\right)\left(\boldsymbol{k}_{3} \cdot \boldsymbol{q}_{1}\right)\left(\boldsymbol{k}_{3} \cdot \boldsymbol{q}_{2}\right)}{q_{1}^{4} q_{2}^{4}} \\
& \quad+5 \text { perm. }
\end{aligned}
$$

$$
\begin{aligned}
B^{(e)} & =-\int \mathrm{d} \boldsymbol{q}_{1} \mathrm{~d} \boldsymbol{q}_{2} \mathrm{~d} \boldsymbol{q}_{3} \delta_{\mathrm{D}}\left(\boldsymbol{q}_{1}+\boldsymbol{q}_{2}-\boldsymbol{k}_{1}\right) \\
& \times \delta_{\mathrm{D}}\left(\boldsymbol{q}_{1}+\boldsymbol{q}_{3}+\boldsymbol{k}_{2}\right) P_{\mathrm{L}}\left(q_{1}\right) P_{\mathrm{L}}\left(q_{2}\right) P_{\mathrm{L}}\left(q_{3}\right) \\
& \times \frac{\left(\boldsymbol{k}_{1} \cdot \boldsymbol{q}_{1}\right)\left(\boldsymbol{k}_{1} \cdot \boldsymbol{q}_{2}\right)\left(\boldsymbol{k}_{2} \cdot \boldsymbol{q}_{1}\right)\left(\boldsymbol{k}_{2} \cdot \boldsymbol{q}_{3}\right)\left(\boldsymbol{k}_{3} \cdot \boldsymbol{q}_{2}\right)\left(\boldsymbol{k}_{3} \cdot \boldsymbol{q}_{3}\right)}{q_{1}^{4} q_{2}^{4} q_{3}^{4}} .
\end{aligned}
$$

Moreover, we can gather diagrams (b) and (c), which have the same form, as

$$
\begin{gathered}
B^{(b)}+B^{(c)}=-P_{\mathrm{L}}\left(k_{2}\right) P_{\mathrm{L}}\left(k_{3}\right) \frac{\left(\boldsymbol{k}_{1} \cdot \boldsymbol{k}_{2}\right)\left(\boldsymbol{k}_{1} \cdot \boldsymbol{k}_{3}\right)}{2 k_{2}^{2} k_{3}^{2}} \int \mathrm{d} \boldsymbol{q} P_{\mathrm{L}}(q) \\
\times \frac{\left(\boldsymbol{k}_{1} \cdot \boldsymbol{q}\right)^{2}+\left(\boldsymbol{k}_{2} \cdot \boldsymbol{q}\right)^{2}+\left(\boldsymbol{k}_{3} \cdot \boldsymbol{q}\right)^{2}}{q^{4}}+2 \text { perm. }
\end{gathered}
$$

We can check that for a CDM-like linear power spectrum, with $P_{\mathrm{L}}(k) \propto k$ for $k \rightarrow 0$ and $P_{\mathrm{L}}(k) \propto k^{-3}$ for $k \rightarrow \infty$, all contributions to the power spectrum and to the bispectrum converge. More precisely, if we define the local spectral index $n$ as

$n(k)=\frac{\mathrm{d} \ln P_{\mathrm{L} 0}(k)}{\mathrm{d} \ln k}$

we see that the one-loop contributions to the power spectrum and the bispectrum converge if $n(0)>-1$ and $n(\infty)<-1$. However, as is well-known (Vishniac 1983; Jain \& Bertschinger 1996), the infrared divergences at $q \rightarrow 0$ cancel out and the sum of all contributions is finite for $n(0)>-3$. This can be easily checked from the explicit expressions (90)-(96). On the other hand, the time-dependence of various contributions is

$P^{\text {tree }} \propto D^{2} \sigma_{8}^{2}, \quad P^{1 \text { loop }} \propto D^{4} \sigma_{8}^{4}$

$B^{\text {tree }} \propto D^{4} \sigma_{8}^{4}, \quad B^{1 \text { loop }} \propto D^{6} \sigma_{8}^{6}$

where $\sigma_{8}$ is the normalization of the linear power spectrum today at $z=0$ (defined as usual from the rms linear density contrast within a sphere of radius $8 h^{-1} \mathrm{Mpc}$ ). Thus, we have the scaling $P_{\mathrm{L}} \propto D^{2} \sigma_{8}^{2}$ for a fixed shape of the linear power spectrum. Higher-order terms scale as higher powers of $P_{\mathrm{L}}$ hence of $D^{2} \sigma_{8}^{2}$.

\subsection{Expansion over cubic interaction}

We now turn to the method presented in Sect. 4, where we expand the path integral over the interaction vertex $K_{\mathrm{s}}$. As explained in Sect. 4, this method exactly recovers the results of the standard perturbation theory of Sect. 7.1.

First, as discussed in Sect. 4, for the nonlinear power spectrum the diagrams (b) and (c) of Fig. 5 give back the contributions of diagram (b) and (c) of Fig. 2 associated with the standard perturbative expansion. Note that one can see at once that there is no mixing of diagrams as one goes from one expansion to the other one since it is clear that both diagrams (b) contain a fixed factor $P_{\mathrm{L}}(k)$ whereas both diagrams (c) contain a double integral over $P_{\mathrm{L}}\left(q_{1}\right) P_{\mathrm{L}}\left(q_{2}\right)$ (hence there is a one-to-one correspondence). In any case, a simple explicit computation of the diagrams of Fig. 5 gives indeed Eq. (90) for the Zeldovich dynamics. Therefore, the conditions of convergence of various integrals are the same as in Sect. 7.1.

We now consider the matter bispectrum. At tree-order, the diagram (a) of Fig. 6 gives back the diagram (a) of Fig. 3. Thus, the explicit computation of Eq. (65) recovers Eq. (91) for the Zeldovich dynamics. At one-loop order, we no longer have such a one-to-one correspondence between both expansions since we have eight one-loop diagrams in Fig. 6 and only four one-loop diagrams in Fig. 3. First, let us consider the contributions which have the form of a triple integration over the linear power spectrum, as in Eq. (95) associated with diagram (e) of Fig. 3. One can see at once that only diagram (f) of Fig. 6 has this form and its explicit calculation gives back Eq. (95). Next, contributions 
with a double integration, as in Eq. (94), arise from diagrams (b) and $(\mathrm{g})$ which read as

$$
\begin{aligned}
& B^{(b)}=-P_{\mathrm{L}}\left(k_{1}\right) \int \mathrm{d} \boldsymbol{q}_{1} \mathrm{~d} \boldsymbol{q}_{2} \delta_{\mathrm{D}}\left(\boldsymbol{q}_{1}+\boldsymbol{q}_{2}-\boldsymbol{k}_{2}\right) \\
& \times \frac{2\left(\boldsymbol{k}_{1} \cdot \boldsymbol{k}_{3}\right)\left(\boldsymbol{k}_{2} \cdot \boldsymbol{q}_{1}\right)\left(\boldsymbol{k}_{2} \cdot \boldsymbol{q}_{2}\right)+\left(2 k_{1}^{2}\left(\boldsymbol{k}_{2} \cdot \boldsymbol{k}_{3}\right)-k_{3}^{2}\left(\boldsymbol{k}_{1} \cdot \boldsymbol{k}_{2}\right)\right)\left(\boldsymbol{q}_{1} \cdot \boldsymbol{q}_{2}\right)}{12 k_{1}^{2} q_{1}^{2} q_{2}^{2}} \\
& \quad \times \frac{\left(\boldsymbol{k}_{2} \cdot \boldsymbol{q}_{1}\right)\left(\boldsymbol{k}_{2} \cdot \boldsymbol{q}_{2}\right)}{q_{1}^{2} q_{2}^{2}} P_{\mathrm{L}}\left(q_{1}\right) P_{\mathrm{L}}\left(q_{2}\right)+5 \text { perm. }
\end{aligned}
$$

and

$$
\begin{aligned}
B^{(g)} & =-P_{\mathrm{L}}\left(k_{1}\right) \int \mathrm{d} \boldsymbol{q}_{1} \mathrm{~d} \boldsymbol{q}_{2} \delta_{\mathrm{D}}\left(\boldsymbol{q}_{1}+\boldsymbol{q}_{2}-\boldsymbol{k}_{2}\right) P_{\mathrm{L}}\left(q_{1}\right) P_{\mathrm{L}}\left(q_{2}\right) \\
& \times\left[\frac{2 k_{1}^{2} q_{1}^{2}\left(\boldsymbol{k}_{3} \cdot \boldsymbol{q}_{2}\right)+2\left(\boldsymbol{k}_{1} \cdot \boldsymbol{q}_{1}\right)\left(\boldsymbol{k}_{3} \cdot \boldsymbol{q}_{2}\right)^{2}}{6 k_{1}^{2} q_{1}^{2} q_{2}^{2}}\right. \\
& \left.+\frac{\left(\boldsymbol{k}_{1} \cdot \boldsymbol{q}_{1}\right)\left[-k_{3}^{2} q_{2}^{2}+\left(k_{1}^{2}+2 k_{3}^{2}+q_{1}^{2}-q_{2}^{2}\right)\left(\boldsymbol{k}_{3} \cdot \boldsymbol{q}_{2}\right)\right]}{6 k_{1}^{2} q_{1}^{2} q_{2}^{2}}\right] \\
& \times \frac{\left(\boldsymbol{k}_{2} \cdot \boldsymbol{q}_{1}\right)\left(\boldsymbol{k}_{2} \cdot \boldsymbol{q}_{2}\right)}{q_{1}^{2} q_{2}^{2}}+5 \text { perm. }
\end{aligned}
$$

Then, summing $B^{(b)}+B^{(g)}$ and writing the result in a symmetric form over $\left\{\boldsymbol{q}_{1}, \boldsymbol{q}_{2}\right\}$ we recover the contribution of Eq. (94). Finally, contributions with a single integration, as in Eq. (96), arise from diagrams (c), (d), (e), (h) and (i), which read as

$$
\begin{aligned}
& B^{(c)}=-P_{\mathrm{L}}\left(k_{2}\right) P_{\mathrm{L}}\left(k_{3}\right) \frac{\left(\boldsymbol{k}_{1} \cdot \boldsymbol{k}_{2}\right)\left(\boldsymbol{k}_{1} \cdot \boldsymbol{k}_{3}\right)}{2 k_{2}^{2} k_{3}^{2}} \int \mathrm{d} \boldsymbol{q} P_{\mathrm{L}}(q) \\
& \times \frac{\left(\boldsymbol{k}_{2} \cdot \boldsymbol{q}\right)^{2}+\left(\boldsymbol{k}_{3} \cdot \boldsymbol{q}\right)^{2}}{q^{4}}+2 \text { perm. }
\end{aligned}
$$

$$
\begin{aligned}
& B^{(d)}=-P_{\mathrm{L}}\left(k_{2}\right) P_{\mathrm{L}}\left(k_{3}\right) \frac{3 k_{2}^{2} k_{3}^{2}+2\left(k_{2}^{2}+k_{3}^{2}\right)\left(\boldsymbol{k}_{2} \cdot \boldsymbol{k}_{3}\right)+\left(\boldsymbol{k}_{2} \cdot \boldsymbol{k}_{3}\right)^{2}}{12 k_{2}^{2} k_{3}^{2}} \\
& \times \int \mathrm{d} \boldsymbol{q} P_{\mathrm{L}}(q) \frac{\left(\boldsymbol{k}_{2} \cdot \boldsymbol{q}\right)^{2}+\left(\boldsymbol{k}_{3} \cdot \boldsymbol{q}\right)^{2}}{q^{4}}+2 \text { perm. }, \\
& B^{(e)=}-P_{\mathrm{L}}\left(k_{2}\right) P_{\mathrm{L}}\left(k_{3}\right) \frac{2 k_{2}^{2} k_{3}^{2}+\left(3 k_{1}^{2}+k_{2}^{2}+k_{3}^{2}\right)\left(\boldsymbol{k}_{2} \cdot \boldsymbol{k}_{3}\right)}{24 k_{2}^{2} k_{3}^{2}} \\
& \quad \times \int \mathrm{d} \boldsymbol{q} P_{\mathrm{L}}(q) \frac{\left(\boldsymbol{k}_{1} \cdot \boldsymbol{q}\right)^{2}}{q^{4}}+2 \text { perm. },
\end{aligned}
$$$$
B^{(h)}=P_{\mathrm{L}}\left(k_{2}\right) P_{\mathrm{L}}\left(k_{3}\right) \int \mathrm{d} \boldsymbol{q} P_{\mathrm{L}}(q)\left\{\frac { ( \boldsymbol { k } _ { 2 } \cdot \boldsymbol { q } ) ( \boldsymbol { k } _ { 3 } \cdot \boldsymbol { q } ) } { 1 2 k _ { 2 } ^ { 2 } k _ { 3 } ^ { 2 } } \left[3 \frac{\left(\boldsymbol{k}_{1} \cdot \boldsymbol{q}\right)^{2}}{q^{4}}\right.\right.
$$$$
\left.-2 \frac{k_{1}^{2} q^{2}+3 k_{2}^{2} k_{3}^{2}+2\left(k_{2}^{2}+k_{3}^{2}\right)\left(\boldsymbol{k}_{2} \cdot \boldsymbol{k}_{3}\right)+\left(\boldsymbol{k}_{2} \cdot \boldsymbol{k}_{3}\right)^{2}}{q^{4}}\right]
$$$$
\left.-\frac{3 q^{2}\left(\boldsymbol{k}_{1} \cdot \boldsymbol{q}\right)\left[k_{3}^{2}\left(\boldsymbol{k}_{2} \cdot \boldsymbol{q}\right)+k_{2}^{2}\left(\boldsymbol{k}_{3} \cdot \boldsymbol{q}\right)\right]}{12 k_{2}^{2} k_{3}^{2} q^{4}}\right\}+2 \text { perm. }
$$

and

$$
\begin{aligned}
& B^{(i)}=P_{\mathrm{L}}\left(k_{2}\right) P_{\mathrm{L}}\left(k_{3}\right) \int \mathrm{d} \boldsymbol{q} P_{\mathrm{L}}(q)\left[\frac{\left(\boldsymbol{k}_{1} \cdot \boldsymbol{q}\right)\left(\boldsymbol{k}_{2} \cdot \boldsymbol{q}\right) k_{3}^{2}\left(2 k_{2}^{2}+3 q^{2}\right)}{12 k_{2}^{2} k_{3}^{2} q^{4}}\right. \\
& +\frac{\left(\boldsymbol{k}_{1} \cdot \boldsymbol{q}\right)\left(\boldsymbol{k}_{3} \cdot \boldsymbol{q}\right) k_{2}^{2}\left(2 k_{3}^{2}+3 q^{2}\right)-\left(\boldsymbol{k}_{1} \cdot \boldsymbol{q}\right)^{2}\left(\boldsymbol{k}_{2} \cdot \boldsymbol{k}_{3}\right)\left(k_{1}^{2}+k_{2}^{2}+k_{3}^{2}\right)}{12 k_{2}^{2} k_{3}^{2} q^{4}} \\
& \left.+\frac{\left(\boldsymbol{k}_{2} \cdot \boldsymbol{q}\right)\left(\boldsymbol{k}_{3} \cdot \boldsymbol{q}\right)\left[2 k_{1}^{2} q^{2}-3\left(\boldsymbol{k}_{1} \cdot \boldsymbol{q}\right)^{2}\right]}{12 k_{2}^{2} k_{3}^{2} q^{4}}\right]+2 \text { perm. }
\end{aligned}
$$

where we used the symmetries $\boldsymbol{q} \leftrightarrow-\boldsymbol{q}$ and $\boldsymbol{k}_{2} \leftrightarrow \boldsymbol{k}_{3}$, and the property $\boldsymbol{k}_{1}+\boldsymbol{k}_{2}+\boldsymbol{k}_{3}=0$, to simplify the expressions. Then, we can check again that the sum $B^{(c)}+B^{(d)}+B^{(e)}+B^{(h)}+B^{(i)}$ of Eqs. (102)-(106) gives back Eq. (96) obtained from the standard perturbative expansion. This consistency check allows us to check these expressions obtained through two different methods and to track possible mistakes in the computations.

We again find that all one-loop contributions to the bispectrum converge if $n(0)>-1$ and $n(\infty)<-1$, except for $B^{(h)}$ and $B^{(i)}$ which require $n(\infty)<-3$. As for the standard perturbative expansion (which we recover by summing all terms) infrared divergences compensate so that we only need $n(0)>-3$. On the other hand, the UV divergences of $B^{(h)}$ and $B^{(i)}$ also compensate so that we recover the standard constraint $n(\infty)<-1$. Thus, although the standard expansion of Sect. 7.1 and the expansion over $K_{\mathrm{s}}$ of this section are actually identical, the contributions obtained at each order are split in different ways between different diagrams so that the convergence properties of individual parts can be different for the two schemes. In particular, we have seen that for the Zeldovich dynamics one obtains an artificial UV divergence for $-3 \leq n(\infty)<-1$ which disappears by summing all diagrams. For numerical purposes, since the contributions (h) and (i) of Eqs. (105), (106) are not well-defined because of this UV divergence, we introduce the regularized contributions $B_{R}^{(h)}$ and $B_{\mathrm{R}}^{(i)}$. They are equal to $B^{(h)}$ and $B^{(i)}$ from which we subtract the UV divergent part, that is, we remove the part that scales as $q^{0}$ from the terms in the brackets in Eqs. (105), (106). This only leaves terms that scale as $q^{-2}$ and gives

$$
\begin{aligned}
B_{R}^{(h)} & =-P_{\mathrm{L}}\left(k_{2}\right) P_{\mathrm{L}}\left(k_{3}\right) \frac{3 k_{2}^{2} k_{3}^{2}+2\left(k_{2}^{2}+k_{3}^{2}\right)\left(\boldsymbol{k}_{2} \cdot \boldsymbol{k}_{3}\right)+\left(\boldsymbol{k}_{2} \cdot \boldsymbol{k}_{3}\right)^{2}}{6 k_{2}^{2} k_{3}^{2}} \\
& \times \int \mathrm{d} \boldsymbol{q} P_{\mathrm{L}}(q) \frac{\left(\boldsymbol{k}_{2} \cdot \boldsymbol{q}\right)\left(\boldsymbol{k}_{3} \cdot \boldsymbol{q}\right)}{q^{4}}+2 \text { perm. }
\end{aligned}
$$

and

$$
\begin{aligned}
B_{R}^{(i)} & =-P_{\mathrm{L}}\left(k_{2}\right) P_{\mathrm{L}}\left(k_{3}\right) \frac{2 k_{2}^{2} k_{3}^{2}+\left(\boldsymbol{k}_{2} \cdot \boldsymbol{k}_{3}\right)\left(k_{1}^{2}+k_{2}^{2}+k_{3}^{2}\right)}{12 k_{2}^{2} k_{3}^{2}} \\
& \times \int \mathrm{d} \boldsymbol{q} P_{\mathrm{L}}(q) \frac{\left(\boldsymbol{k}_{1} \cdot \boldsymbol{q}\right)^{2}}{q^{4}}+2 \text { perm. }
\end{aligned}
$$

Since the UV divergences of $B^{(h)}$ and $B^{(i)}$ compensate we have $B^{(h)}+B^{(i)}=B_{R}^{(h)}+B_{R}^{(i)}$ and we can use $B_{R}^{(h)}$ and $B_{\mathrm{R}}^{(i)}$ instead of $B^{(h)}$ and $B^{(i)}$.

\subsection{Direct steepest-descent method}

We now consider the direct steepest-descent method presented in Sect. 5.1. First, the two-point functions $C$ and $R$ are obtained from the diagrams of Figs. 8, 9 that correspond to Eqs. (71), (73). Thus, in terms of nonlinear two-point functions we have only one one-loop diagram for either $C$ or $R$, which corresponds to an infinite series over the linear two-point functions $C_{\mathrm{L}}$ and $R_{\mathrm{L}}$. The results obtained at one-loop order have been described in detail in Valageas (2007b). Thus, the nonlinear response $R$ reads as

$R\left(x_{1}, x_{2}\right)=R_{\mathrm{L}}\left(x_{1}, x_{2}\right) \cos \left[\omega(k)\left(D_{1}-D_{2}\right)\right]$,

where $R_{\mathrm{L}}$ is the linear response given in Eq. (19), $D=\mathrm{e}^{\eta}$ is the linear growth factor and $\omega(k)$ (where $k=k_{1}=k_{2}$ is the wavenumber) is given by

$\omega(k)=k \sigma_{v}$. 
Here $\sigma_{v}^{2}$ is the variance of the one-dimensional linear displacement field $s_{\mathrm{L} 0}$ given in Eq. (83). Note that the nonlinear response $R$ only depends on the linear power spectrum through this velocity variance. From the expression of the linear response $R_{\mathrm{L}}$, Eq. (109) gives

$$
\begin{aligned}
R\left(k ; D_{1}, D_{2}\right)= & \theta\left(D_{1}-D_{2}\right) \cos \left[\omega(k)\left(D_{1}-D_{2}\right)\right] \\
& \times \sum_{\ell=1}^{2}\left(\frac{D_{1}}{D_{2}}\right)^{\alpha_{\ell}} R_{(\ell)},
\end{aligned}
$$

with

$\alpha_{1}=1, \quad \alpha_{2}=0$,

and the two constant matrices $R_{(1)}$ and $R_{(2)}$, associated with the linear growing and decaying (here constant) modes are

$R_{(1)}=\left(\begin{array}{ll}0 & 1 \\ 0 & 1\end{array}\right)$ and $R_{(2)}=\left(\begin{array}{cc}1 & -1 \\ 0 & 0\end{array}\right)$.

The expression of the two-point correlation $C$ is more intricate and it depends on the details of the linear power spectrum. From Eq. (109) the first term of Eq. (73) reads exactly as (Valageas 2007b)

$R \times C_{\mathrm{L}}\left(\eta_{\mathrm{I}}\right) \times R^{\mathrm{T}}=C_{\mathrm{L}}\left(x_{1}, x_{2}\right) \cos \left(\omega D_{1}\right) \cos \left(\omega D_{2}\right)$.

The second term of Eq. (73) has a more intricate structure but we shall use the qualitative approximation

R.П.R $R^{\mathrm{T}} \simeq C_{\mathrm{L}}\left(x_{1}, x_{2}\right) \sin \left(\omega D_{1}\right) \sin \left(\omega D_{2}\right)$.

As seen in Valageas (2007b), the exact one-loop result contains an additional term but it has no qualitative effect on $C$ hence we shall use the simple approximation obtained from the sum of Eqs. (114), (115),

$C\left(x_{1}, x_{2}\right) \simeq C_{\mathrm{L}}\left(x_{1}, x_{2}\right) \cos \left[\omega(k)\left(D_{1}-D_{2}\right)\right]$,

which gives:

$C\left(k ; D_{1}, D_{2}\right)=D_{1} D_{2} \cos \left[\omega(k)\left(D_{1}-D_{2}\right)\right] P_{\mathrm{L} 0}(k)\left(\begin{array}{ll}1 & 1 \\ 1 & 1\end{array}\right)$.

Equations (109), (116), also correspond to a simple effective dynamics where the nonlinearity is reduced to a simple nonGaussian random advection by a large-scale flow which gives rise to the cosine factors (Valageas 2007a,b). Then, these cosine factors simply describe the (weak) decorrelation, or loss of memory, due to this random advection (compare with Eqs. (82), (86)). Note that the time dependence obtained from the steepestdescent method is quite different from the one obtained in Eq. (98) since Eqs. (114), (115) give for the equal-time power spectrum

$P^{\text {tree }} \sim D^{2} \sigma_{8}^{2}, \quad P^{1 \text { loop }} \sim D^{2} \sigma_{8}^{2}$,

where we label as the "tree" contribution the first diagram of Fig. 9, associated with Eq. (114), and as the "1loop" contribution the second diagram of Fig. 9, associated with Eq. (115). Therefore, we now find that both contributions exhibit the same scaling in the nonlinear regime and they only grow as $D^{2} \sigma_{8}^{2}$ instead of $D^{4} \sigma_{8}^{4}$ as in Eq. (98).

The three-point correlation is now given up to one-loop order by the five diagrams of Fig. 10. As explained in Sect. 5.1, this simplification with respect to the expansion over powers of $K_{\mathrm{s}}$, studied in Sects. 4 and 7.2, comes from the fact that oneloop diagrams associated with a "renormalization" of the twopoint functions $C$ and $R$ are included in the tree-diagram (a) of Fig. 10 and only the four diagrams associated with the "renormalization" of the three-point vertex $K_{\mathrm{s}}$ are new structures with respect to the previous order (tree-order). Let us first consider the tree-diagram (a). Its explicit expression is of the form of Eq. (65) but using the nonlinear two-point functions $R$ and $C$. From Eqs. (111), (117), we see that the integral over time $\eta_{1}^{\prime}$ in Eq. (65) gives rise to the two quantities (for $\ell=1,2$ ):

$$
\begin{gathered}
\int_{0}^{D} \frac{\mathrm{d} D_{1}^{\prime}}{D_{1}^{\prime}}\left(\frac{D}{D_{1}^{\prime}}\right)^{\alpha_{\ell}} D^{2} D_{1}^{\prime 2} \prod_{j=1}^{3} \cos \left[\omega_{j}\left(D-D_{1}^{\prime}\right)\right] \\
=\frac{D^{4}}{2-\alpha_{\ell}} T_{(\ell)}^{(a)}\left(\omega_{j} D\right) .
\end{gathered}
$$

This defines the two time-dependent functions $T_{(\ell)}^{(a)}$ of the three variables $\omega_{j} D$, associated with diagram (a). In Eq. (119) we considered the case of equal-time statistics $D_{1}=D_{2}=D_{3}=D$ and we introduced:

$\omega_{j}=\omega\left(k_{j}\right)=k_{j} \sigma_{v}$.

We also normalized $T_{(\ell)}^{(a)}$ so that for $\omega_{j}=0$ we have $T_{(\ell)}^{(a)}\left(\omega_{j}=\right.$ $0)=1$. Thus, by putting $T_{(\ell)}^{(a)}=1$ we must recover the result (91) obtained by integrating Eq. (65) over the linear twopoint functions, as in the expansion scheme of Sect. 7.2 where we expanded over powers of $K_{\mathrm{s}}$. This provides a convenient consistency check. Note that all the time-dependence is included in the standard prefactor $D^{4}$ and the function $T_{(\ell)}^{(a)}$, which only depends on the linear power spectrum and on the wavenumbers $k_{j}$ through the three combinations $k_{j} \sigma_{v}$. The explicit expressions of $T_{(\ell)}^{(a)}$ are

$$
\begin{aligned}
& T_{(1)}^{(a)}=\frac{1}{4}\left\{\operatorname{sinc}\left[D\left(-\omega_{1}+\omega_{2}+\omega_{3}\right)\right]+\operatorname{sinc}\left[D\left(\omega_{1}-\omega_{2}+\omega_{3}\right)\right]\right. \\
& \left.+\operatorname{sinc}\left[D\left(\omega_{1}+\omega_{2}-\omega_{3}\right)\right]+\operatorname{sinc}\left[D\left(\omega_{1}+\omega_{2}+\omega_{3}\right)\right]\right\}
\end{aligned}
$$

and

$$
\begin{aligned}
& T_{(2)}^{(a)}=\frac{1}{4}\left\{\operatorname{cosc}\left[D\left(-\omega_{1}+\omega_{2}+\omega_{3}\right)\right]+\operatorname{cosc}\left[D\left(\omega_{1}-\omega_{2}+\omega_{3}\right)\right]\right. \\
& \left.+\operatorname{cosc}\left[D\left(\omega_{1}+\omega_{2}-\omega_{3}\right)\right]+\operatorname{cosc}\left[D\left(\omega_{1}+\omega_{2}+\omega_{3}\right)\right]\right\}
\end{aligned}
$$

where we introduced the functions

$\operatorname{sinc}(x)=\frac{\sin (x)}{x}, \quad \operatorname{cosc}(x)=\frac{2(1-\cos (x))}{x^{2}}=\operatorname{sinc}^{2}\left(\frac{x}{2}\right)$.

We can check that $T_{(\ell)}^{(a)}(0)=1$, and in the highly nonlinear regime we have

$D \omega_{j} \gg 1: \quad T_{(1)}^{(a)} \sim \frac{1}{D \omega}, \quad T_{(2)}^{(a)} \sim \frac{1}{(D \omega)^{2}}$,

(with $\left|\sum \pm \omega_{j}\right| \sim \omega$ ). Then, diagram (a) of Fig. 10 reads as

$\begin{aligned} B^{(a)}= & P_{\mathrm{L}}\left(k_{2}\right) P_{\mathrm{L}}\left(k_{3}\right) \frac{T_{(1)}^{(a)} k_{1}^{2}\left(\boldsymbol{k}_{2} \cdot \boldsymbol{k}_{3}\right)+T_{(2)}^{(a)}\left[k_{2}^{2} k_{3}^{2}-\left(\boldsymbol{k}_{2} \cdot \boldsymbol{k}_{3}\right)^{2}\right]}{k_{2}^{2} k_{3}^{2}} \\ & +2 \text { perm. }\end{aligned}$ 
We can check that by substituting $T_{(\ell)}^{(a)}=1$ (and using $\boldsymbol{k}_{1}+\boldsymbol{k}_{2}+$ $\boldsymbol{k}_{3}=0$ ) we recover the standard tree-order contribution (91). Moreover, in the nonlinear regime we have the scaling

$D \omega_{j} \gg 1: \quad B^{(a)} \sim \frac{D^{4} \sigma_{8}^{4}}{D \omega} \propto D^{3} \sigma_{8}^{3}$,

since $\omega \propto \sigma_{8}$. Thus, the nonlinear corrections resummed in the large- $N$ steepest-descent method have modified the scaling over $D$ of the tree diagram (a) as compared with the standard perturbation theory result (91) which scales as the first term in (99).

Next, let us consider the first one-loop diagram (f) of Fig. 10. We can again factorize the time-dependent parts which read as

$$
\begin{aligned}
\int_{0}^{D} & \prod_{j=1}^{3} \frac{\mathrm{d} D_{j}^{\prime}}{D_{j}^{\prime}}\left(\frac{D}{D_{j}^{\prime}}\right)^{\alpha_{\ell_{j}}} D_{j}^{\prime 2} \cos \left[\omega_{j}\left(D-D_{j}^{\prime}\right)\right] \\
& =\frac{D^{6}}{\left(2-\alpha_{\ell_{1}}\right)\left(2-\alpha_{\ell_{2}}\right)\left(2-\alpha_{\ell_{3}}\right)} T_{\left(\ell_{1}, \ell_{2}, \ell_{3}\right)}^{(f)}\left(\omega_{j} D\right) .
\end{aligned}
$$

This defines the functions $T_{\left(\ell_{j}\right)}^{(f)}$ of the three variables $\omega_{j} D$. They are parameterized by the indices $\ell_{j}$ of the response matrices $R_{\left(\ell_{j}\right)}$ of Eq. (113). Since the diagram (f) involves three response functions, there are $2^{3}=8$ such functions $T_{\left(\ell_{j}\right)}^{(f)}$. However, thanks to the symmetry of diagram (f), $T_{\left(\ell_{j}\right)}^{(f)}$ can be split into a product of three terms as

$T_{\left(\ell_{1}, \ell_{2}, \ell_{3}\right)}^{(f)}\left(\omega_{j} D\right)=T_{\left(\ell_{1}\right)}^{(f)}\left(\omega_{1} D\right) T_{\left(\ell_{2}\right)}^{(f)}\left(\omega_{2} D\right) T_{\left(\ell_{3}\right)}^{(f)}\left(\omega_{3} D\right)$,

with

$T_{(\ell)}^{(f)}(\omega D)=\left(2-\alpha_{\ell}\right) \int_{0}^{1} \frac{\mathrm{d} x}{x} x^{2-\alpha_{\ell}} \cos [\omega D(1-x)]$.

The integration over $x$ yields

$T_{(1)}^{(f)}(\omega D)=\operatorname{sinc}(\omega D), \quad T_{(2)}^{(f)}(\omega D)=\operatorname{cosc}(\omega D)$,

which gives the nonlinear scaling

$D \omega_{j} \gg 1: \quad T_{(1,1,1)}^{(f)}\left(\omega_{j} D\right) \sim \frac{1}{(D \omega)^{3}}$,

and higher powers of $1 /(D \omega)$ arise if some indices $\ell_{j}$ are equal to 2 . Then, diagram (f) reads as

$$
\begin{aligned}
& B^{(f)}=\int \mathrm{d} \boldsymbol{q}_{1} \mathrm{~d} \boldsymbol{q}_{2} \mathrm{~d} \boldsymbol{q}_{3} \delta_{\mathrm{D}}\left(\boldsymbol{q}_{1}+\boldsymbol{q}_{2}-\boldsymbol{k}_{1}\right) \delta_{\mathrm{D}}\left(\boldsymbol{q}_{1}+\boldsymbol{q}_{3}+\boldsymbol{k}_{2}\right) \\
& \times \frac{T_{(1)}^{(f)}\left(\omega_{1} D\right) k_{1}^{2}\left(\boldsymbol{q}_{1} \cdot \boldsymbol{q}_{2}\right)+T_{(2)}^{(f)}\left(\omega_{1} D\right)\left[q_{1}^{2} q_{2}^{2}-\left(\boldsymbol{q}_{1} \cdot \boldsymbol{q}_{2}\right)^{2}\right]}{q_{1}^{2} q_{2}^{2}} \\
& \times \frac{T_{(1)}^{(f)}\left(\omega_{2} D\right) k_{2}^{2}\left(\boldsymbol{q}_{1} \cdot \boldsymbol{q}_{3}\right)+T_{(2)}^{(f)}\left(\omega_{2} D\right)\left[q_{1}^{2} q_{3}^{2}-\left(\boldsymbol{q}_{1} \cdot \boldsymbol{q}_{3}\right)^{2}\right]}{q_{1}^{2} q_{3}^{2}} \\
& \times \frac{-T_{(1)}^{(f)}\left(\omega_{3} D\right) k_{3}^{2}\left(\boldsymbol{q}_{2} \cdot \boldsymbol{q}_{3}\right)+T_{(2)}^{(f)}\left(\omega_{3} D\right)\left[q_{2}^{2} q_{3}^{2}-\left(\boldsymbol{q}_{1} \cdot \boldsymbol{q}_{3}\right)^{2}\right]}{q_{2}^{2} q_{3}^{2}} \\
& \times P_{\mathrm{L}}\left(q_{1}\right) P_{\mathrm{L}}\left(q_{2}\right) P_{\mathrm{L}}\left(q_{3}\right) .
\end{aligned}
$$

We can check that by substituting $T_{(\ell)}^{(f)}=1$ we again recover the corresponding one-loop diagram (f) of the expansion over $K_{\mathrm{S}}$ of Sect. 7.2, which is also given by Eq. (95) obtained from the standard perturbative expansion of Sect. 7.1. Then, we obtain for this one-loop diagram (f) the scaling

$D \omega_{j} \gg 1: \quad B^{(f)} \sim \frac{D^{6} \sigma_{8}^{6}}{(D \omega)^{3}} \propto D^{3} \sigma_{8}^{3}$.
Thus, the scaling of this one-loop diagram is again modified as compared with the corresponding one-loop diagram obtained in the standard perturbation theory, which was equal to Eq. (95) and which scaled as the second term of (99). Moreover, contrary to the standard expansion scheme, the one-loop diagram shows the same scaling as the tree diagram (compare with Eq. (126)).

The three other one-loop diagrams of Fig. 10 can be computed in the same fashion. In particular, because of the triple integration over inner times $D_{j}^{\prime}$, weighted by the factors $\cos \left[\omega_{j}\left(D-D_{j}^{\prime}\right)\right]$ as in Eq. (127), we can check that all timedependent factors $T$ obey the same scaling as in Eq. (131),

$D \omega_{j} \gg 1: \quad T_{\left(\ell_{j}\right)}^{(f, g, h, i)}\left(\omega_{j} D\right) \sim \frac{1}{(D \omega)^{3}}$,

with possible higher powers if some $\ell_{j}$ are equal to 2 . Therefore, all one-loop diagrams scale as Eq. (133) and as the tree diagram of Eq. (126) computed at this same one-loop order. Thus, we recover the nice improvement over the standard perturbation theory already obtained for the two-point correlation: the oneloop correction does not grow with an additional power $D^{2}$ as compared with the tree-order result and seems better behaved. Unfortunately, as shown in Valageas (2007b), at higher orders exponentially growing terms appear within this steepest-descent expansion scheme. Therefore, at two-loop order and beyond, this large- $N$ method becomes even more ill-behaved than the standard expansion in the sense of exhibiting strongly growing highorder terms.

On the other hand, we can note that the growth as $D^{2} \sigma_{8}^{2}$ of the two-point correlation (see Eq. (118)) and as $D^{3} \sigma_{8}^{3}$ of the three-point correlation breaks physical constraints, that is, it cannot be achieved by any matter density distribution. Indeed, the positivity of the matter density $\rho(\boldsymbol{x})$ implies that $C_{\delta 3}$ should grow at least as $\left(C_{\delta 2}\right)^{2}$ in the highly nonlinear regime, see Eq. (6) in Valageas (1999). This implies that the strong constraint $\rho(\boldsymbol{x}) \geq 0$ is not satisfied by the large- $N$ steepest-descent expansion (nor by the usual perturbative expansion). The growth factors $D^{2}$ and $D^{3}$ are rather reminiscent of generic unconstrained fields $\psi$ with a time-dependence which would approximately factorize as $\psi(\boldsymbol{x}, D) \sim D$.

Finally, we must take care of the UV divergences of diagrams (h) and (i) already encountered in Sect. 7.2. In the expansion over $K_{\mathrm{s}}$, described in Sect. 7.2, the UV divergences of diagrams (h) and (i) exactly compensate and we could as well use the regularized contributions $B_{\mathrm{R}}^{(h)}$ and $B_{\mathrm{R}}^{(h)}$ of Eqs. (107), (108). For the steepest-descent scheme this is no longer true because the divergent parts are multiplied by the independent functions $T^{(h)}$ and $T^{(i)}$. On the other hand, at order $P_{\mathrm{L}}^{3}$ we could substitute $T^{(h)}=T^{(i)}=1$ into diagrams (h) and (i), which already involve a product over three terms $P_{\mathrm{L}}$, so that the divergences again compensate. In fact, at order $P_{\mathrm{L}}^{3}$ we must recover the results of the standard expansion scheme hence we know that such a UV divergence can only appear at higher orders (if at all). Therefore, in a fashion similar to the procedure of Sect. 7.2, we define regularized contributions $B_{\mathrm{R}}^{(h)}$ and $B_{\mathrm{R}}^{(i)}$ by subtracting from $B^{(h)}$ and $B^{(i)}$ their UV divergent part. This makes no difference for the sum of contributions (h) and (i) at order $P_{\mathrm{L}}^{3}$ (but we have removed higher-order terms which happened to diverge and did not cancel in the sum). This gives:

$$
\begin{aligned}
B_{R}^{(h)}= & -\frac{T_{(111)}^{(h)} 2\left(\boldsymbol{k}_{2} \cdot \boldsymbol{k}_{3}\right) k_{1}^{2}+T_{(211)}^{(h)} 3\left[k_{2}^{2} k_{3}^{2}-\left(\boldsymbol{k}_{2} \cdot \boldsymbol{k}_{3}\right)^{2}\right]}{6 k_{2}^{2} k_{3}^{2}} \\
& \times P_{\mathrm{L}}\left(k_{2}\right) P_{\mathrm{L}}\left(k_{3}\right) \int \mathrm{d} \boldsymbol{q} P_{\mathrm{L}}(q) \frac{\left(\boldsymbol{k}_{2} \cdot \boldsymbol{q}\right)\left(\boldsymbol{k}_{3} \cdot \boldsymbol{q}\right)}{q^{4}}+2 \text { perm. }
\end{aligned}
$$


and

$$
\begin{aligned}
& B_{R}^{(i)}=-\frac{T_{(111)}^{(i)}\left(\boldsymbol{k}_{2} \cdot \boldsymbol{k}_{3}\right) k_{1}^{2}+T_{(212)}^{(h)}\left[k_{2}^{2} k_{3}^{2}-\left(\boldsymbol{k}_{2} \cdot \boldsymbol{k}_{3}\right)^{2}\right]}{6 k_{2}^{2} k_{3}^{2}} \\
& \times P_{\mathrm{L}}\left(k_{2}\right) P_{\mathrm{L}}\left(k_{3}\right) \int \mathrm{d} \boldsymbol{q} P_{\mathrm{L}}(q) \frac{\left(\boldsymbol{k}_{1} \cdot \boldsymbol{q}\right)^{2}}{q^{4}}+2 \text { perm. }
\end{aligned}
$$

Of course, we can check that by substituting $T^{(h)}=T^{(i)}=1$ into Eqs. (135), (136), and using $\boldsymbol{k}_{1}+\boldsymbol{k}_{2}+\boldsymbol{k}_{3}=0$, we recover the regularized contributions (107)-(108) introduced in Sect. 7.2 for the expansion over $K_{\mathrm{s}}$. On the other hand, this analysis shows that the partial resummations involved in the large- $N$ methods have introduced spurious UV divergences. For the Zeldovich dynamics, we know that such UV divergences are not seen in the exact nonlinear results that can be obtained from the solution (35) of the dynamics. Therefore, they must cancel out by including further diagrams or by expanding back over powers of $P_{\mathrm{L}}$, as in the standard perturbation theory. The fact that they do not cancel out automatically may be seen as a shortcoming of resummations schemes such as these large- $N$ methods. Indeed, the use of partial resummations is intended to improve the behavior of the predicted nonlinear correlations as compared with the results of the standard perturbative expansions. For instance, one would like to recover the damping of the response function in the nonlinear regime and a better control of higher-order terms. At one-loop order this is partly achieved for the bispectrum as all contributions show the same scaling (133) instead of the hierarchy (99). However, this comes at the price of an increased sensitivity to the smallest scales that even leads to a divergence for $n(\infty) \geq-3$. Nevertheless, since this divergence disappears in the expansion over $P_{\mathrm{L}}$ it is always possible to remove it by subtraction as in Eqs. (135), (136).

\section{Gravitational dynamics}

As seen in Sects. 2-5, we can apply to the gravitational dynamics the expansion schemes described in Sect. 7 for the Zeldovich dynamics. We do not give the explicit expressions of various diagrams here as they are rather long, except for the expansion over $K_{\mathrm{S}}$ in the Appendix.

\subsection{Standard perturbation theory}

For the standard perturbation theory recalled in Sect. 3, the kernels $F_{n}$ are computed from recursion relations derived from the equation of motion (8). Following the procedure described in Sect. 3, this gives the well-known results for the matter power spectrum and bispectrum that we do not repeat here (Bernardeau et al. 2002, and references therein; Scoccimarro \& Frieman 1996a,b; Scoccimarro 1997). The conditions of convergence of individual diagrams are the same as for the Zeldovich dynamics of Sect. 7.1, that is, $n(0)>-1$ and $n(\infty)<-1$. Again, since the Galilean invariance applies to both dynamics, the infrared divergences cancel out as we sum all diagrams so that we only require $n(0)>-3$ (Vishniac 1983; Jain \& Bertschinger 1996). Finally, the dependence on the linear growth factor $D(\eta)$ and on the amplitude $\sigma_{8}$ of the linear power spectrum are as in Eqs. (98), (99). We do not plot the results here since they are identical to the results obtained from the expansion over $K_{\mathrm{s}}$ displayed in the next section.

\subsection{Expansion over cubic interaction}

The expansion over powers of $K_{\mathrm{S}}$ proceeds exactly as for the Zeldovich dynamics studied in Sect. 7.2. For the power spectrum there is again a one-to-one correspondence between the diagrams (b) and (c) of Figs. 2 and 5. For the bispectrum, the correspondence is again between the groups of diagrams $\{(a),(b+c),(d),(e)\}$ of Fig. 3 and $\{(a),(c+d+e+h+i),(b+g),(f)\}$ of Fig. 6. For completeness, we give the expressions of these diagrams in the Appendix.

These results are similar to those obtained for the Zeldovich dynamics in Sect. 7.2, except that the rational functions that appear in the integrals are more intricate. In particular, the denominators do not factorize as products of $k_{j}$ and $q_{j}$ so that angular integrations require more care. The conditions of convergence are the same as for the Zeldovich dynamics, that is, $n(0)>-1$ and $n(\infty)<-1$, except for contributions $B^{(h)}$ and $B^{(i)}$ which require $n(\infty)<-3$. Again, the infrared and ultraviolet divergences compensate so that the sum is well-defined for $n(0)>-3$ and $n(\infty)<-1$. As in Eqs. (107)-(108), it is convenient to introduce the regularized contributions $B_{\mathrm{R}}^{(h)}$ and $B_{\mathrm{R}}^{(i)}$ by subtracting from $B^{(h)}$ and $B^{(i)}$ their UV divergent part.

From the bispectrum $B\left(k_{1}, k_{2}, k_{3}\right)$ and the power spectrum $P(k)$ we define as usual the reduced bispectrum $Q\left(k_{1}, k_{2}, k_{3}\right)$ by

$Q\left(k_{1}, k_{2}, k_{3}\right)=\frac{B\left(k_{1}, k_{2}, k_{3}\right)}{P\left(k_{2}\right) P\left(k_{3}\right)+2 \text { perm. }}$

From Eq. (51), in the quasi-linear regime it is independent of time and of the normalization of the linear power spectrum. Let us note $S\left(k_{1}, k_{2}, k_{3}\right)$ the denominator of Eq. (137):

$S\left(k_{1}, k_{2}, k_{3}\right)=P\left(k_{2}\right) P\left(k_{3}\right)+2$ perm.

Within the standard perturbative expansion over powers of $P_{\mathrm{L}}$, or the equivalent expansion over $K_{\mathrm{s}}$, the ratio (137) reads up to one-loop order as

$$
\begin{aligned}
Q & =\frac{B^{\text {tree }}+B^{1 \text { loop }}}{S^{\text {tree }}+S^{1 \text { loop }}} \\
& =\frac{B^{\text {tree }}}{S^{\text {tree }}}+\frac{B^{1 l o o p}}{S^{\text {tree }}}-\frac{B^{\text {tree }}}{S^{\text {tree }}} \frac{S^{1 \text { loop }}}{S^{\text {tree }}}
\end{aligned}
$$

where in the second line we have expanded the ratio $B / S$ of the first line. The various contributions to the bispectrum have been described in the previous sections, whereas the denominator $S$ is given up to one-loop order by

$$
\begin{aligned}
S^{\text {tree }} & =P_{\mathrm{L}}\left(k_{2}\right) P_{\mathrm{L}}\left(k_{3}\right)+2 \text { perm. } \\
S^{1 \text { loop }} & =P_{\mathrm{L}}\left(k_{2}\right) P^{1 \text { loop }}\left(k_{3}\right)+5 \text { perm. }
\end{aligned}
$$

We show in Fig. 15 the results obtained for the reduced bispectrum $Q$ as defined by Eq. (139), that is, we keep the ratio (139) without expanding again as in Eq. (140). We plot $Q$ as a function of wavenumber $k$ for equilateral triangles $k_{1}=k_{2}=k_{3}=k$. The data points are taken from Fosalba et al. (2005). They correspond to a $\Lambda \mathrm{CDM}$ simulation from the Virgo archive at redshift $z=0$. The four dashed curves labelled $\{a, c+d+e+h+i, b+g, f\}$ are the contributions of the various diagrams obtained for $B$ in the expansion over $K_{\mathrm{s}}$. Diagrams of the same form (i.e., that involve the same number of integrations over the linear power spectrum) have been gathered and divided by the denominator $S^{\text {tree }}+S^{1 \text { loop }}$. Their sum is the full reduced bispectrum $Q$ shown by the solid line. The rising dotted line $Q_{\mathrm{PCS}}$ is the phenomenological model 


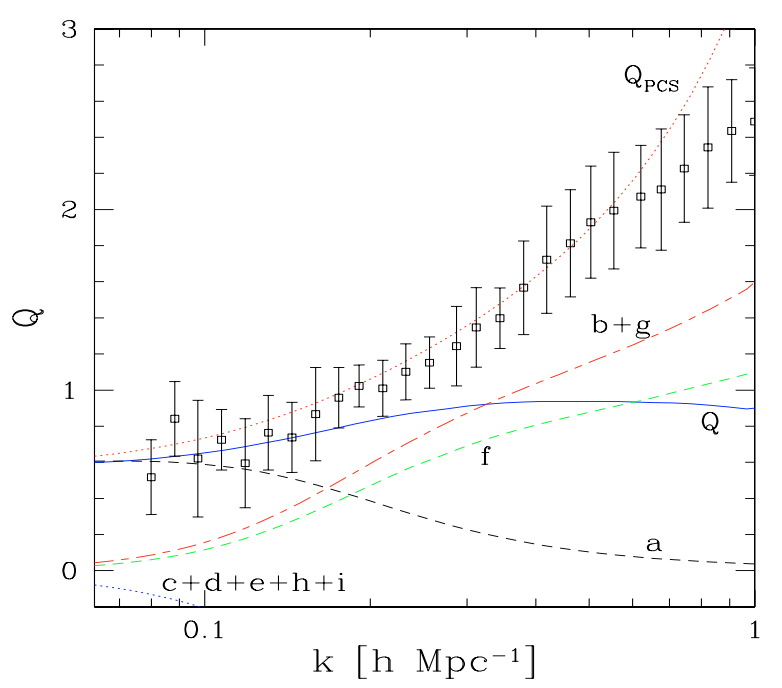

Fig. 15. The reduced bispectrum $Q$ defined at one-loop order by the rational function (139). We show $Q$ as a function of wavenumber $k$ for equilateral triangles $k_{1}=k_{2}=k_{3}=k$. The data points are taken from Fosalba et al. (2005), for a $\Lambda \mathrm{CDM}$ simulation at $z=0$. The letters correspond to the various contributions, such as $B^{(a)} /\left(S^{\text {tree }}+S^{1 \text { loop }}\right)$, associated with tree and one-loop diagrams obtained for the bispectrum $B$ within the expansion over $K_{\mathrm{s}}$. The solid line $Q$ is the full reduced bispectrum $Q$ (i.e. the sum of all these contributions). It is also equal to the prediction of the standard perturbation theory. The rising dotted line $Q_{\mathrm{PCS}}$ is the phenomenological model of Pan et al. (2007).

of Pan et al. (2007). It is based on the scale transformation introduced by Hamilton et al. (1991) and it uses as input the nonlinear power spectrum, obtained from the fit to numerical simulations given by Smith et al. (2003). We show in Fig. 16 the results obtained for the same case when we use the expansion (140) instead of the ratio (139). The new curve labeled $-B S / S^{2}$ is the last term of Eq. (140).

Let us recall here that the predictions for the bispectrum $B$, hence for the reduced bispectrum $Q$, obtained from this expansion over $K_{\mathrm{s}}$, are equal to the results of the standard perturbation theory. Note that the tree-order result, given by

$Q^{\text {tree }}=\frac{B^{\text {tree }}}{S^{\text {tree }}}$,

also corresponds to the horizontal line $(a)$ in Fig. 16. It is constant since both $B^{\text {tree }}$ and $S^{\text {tree }}$ scale as $P_{\mathrm{L}}(k)^{2}$. We can check that the reduced bispectra $Q$ obtained from the two Eqs. (139), (140) agree on quasi-linear scales, up to $0.2 \mathrm{~h} \mathrm{Mpc}^{-1}$, where $\Delta_{L}^{2}(k) \simeq 1$ (here $\Delta_{L}^{2}(k)=4 \pi k^{3} P(k)$ is the power per logarithmic wavenumber). At smaller scales the ratio (139) goes to a constant (that is, a value that is independent of the normalization of $P_{\mathrm{L}}$ but shows a weak dependence on the shape of the linear power spectrum, since both $B^{1 \text { loop }}$ and $S^{1 \text { loop }}$ scale as $P_{\mathrm{L}}^{3}$ ), whereas the expanded version (140) grows as $\Delta_{\mathrm{L}}^{2}$. This leads to a better agreement of Eq. (140) with the numerical results up to $0.4 \mathrm{~h} \mathrm{Mpc}^{-1}$, where $\Delta_{\mathrm{L}}^{2}(k) \simeq 4$. However, it is clear that this better match has no strong foundations. We can also note that in the nonlinear regime there are some cancellations between various contributions, for both Eqs. (139)-(140). On the other hand, it appears that the phenomenological model of Pan et al. (2007) works best, up to $0.8 h \mathrm{Mpc}^{-1}$, where $\Delta_{L}^{2}(k) \simeq 17$. However, at $k \sim 0.1-0.2 h \mathrm{Mpc}^{-1}$ it may slightly overestimate $Q$.

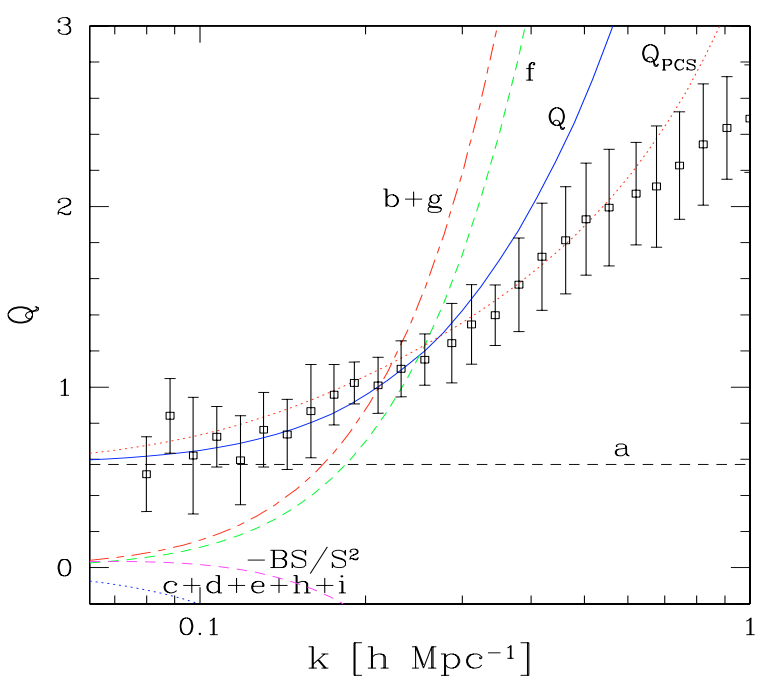

Fig. 16. The reduced bispectrum $Q$ at one-loop order for equilateral triangles as in Fig. 15, but computed from the expansion (140) instead of the ratio (139). The symbols are as in Fig. 15 but the contributions to the bispectrum $B$ associated with tree and one-loop diagrams are now divided by $S^{\text {tree }}$, as in the first two terms of Eq. (140), instead of $\left(S^{\text {tree }}+S^{\text {lloop }}\right)$, and the new curve labeled $-B S / S^{2}$ corresponds to the last term of Eq. (140).

We show in Fig. 17 the reduced bispectrum $Q$ as a function of the angle $\theta$ between the two wavenumbers $\boldsymbol{k}_{1}$ and $\boldsymbol{k}_{2}$ :

$$
\cos (\theta)=\frac{\boldsymbol{k}_{1} \cdot \boldsymbol{k}_{2}}{k_{1} k_{2}}, \quad k_{3}=\sqrt{k_{1}^{2}+k_{2}^{2}+2 k_{1} k_{2} \cos (\theta)} .
$$

In the lower left panel (c) we have $k_{2}=k_{1}$ whereas in other panels $k_{2}=2 k_{1}$. We probe smaller scales farther into the nonlinear regime going from panel (a) to panel (d). On large scales, all curves match the tree-order result and numerical data, as seen in panel (a), and show a strong dependence on the angle $\theta$. On smaller scales, the dependence on $\theta$ decreases, as shown by the simulations. This behavior is captured by the phenomenological model of Pan et al. (2007) but not by the one-loop predictions of Eqs. (139), (140). In agreement with Figs. 15, 16, the ratio (139) yields a smaller value for $Q$ than the expansion (140), but none of these two predictions matches the data for $\Delta_{L}^{2}(k)>0.3$. The results displayed in Figs. 15, 17 agree with previous studies of the standard perturbation theory, discussed for instance in great details in the review of Bernardeau et al. (2002).

\subsection{Direct steepest-descent method}

For the gravitational dynamics, the nonlinear response function is no longer given by Eq. (109) at one-loop order in the steepest-descent expansion. However, Eqs. (109) and (117) still provide a reasonable approximation of the exact one-loop result, as seen in Valageas (2007a). Thus, in this article we use the approximations (109) and (117) to investigate the behavior of the steepest-descent method for the gravitational dynamics as well. Then, from Eq. (19) the powers $\alpha_{\ell}$ and the matrices $R_{(\ell)}$ of Eqs. (112), (113) must be replaced by

$\alpha_{1}=1, \quad \alpha_{2}=-\frac{3}{2}$,

$R_{(1)}=\frac{1}{5}\left(\begin{array}{ll}3 & 2 \\ 3 & 2\end{array}\right), \quad R_{(2)}=\frac{1}{5}\left(\begin{array}{cc}2 & -2 \\ -3 & 3\end{array}\right)$. 

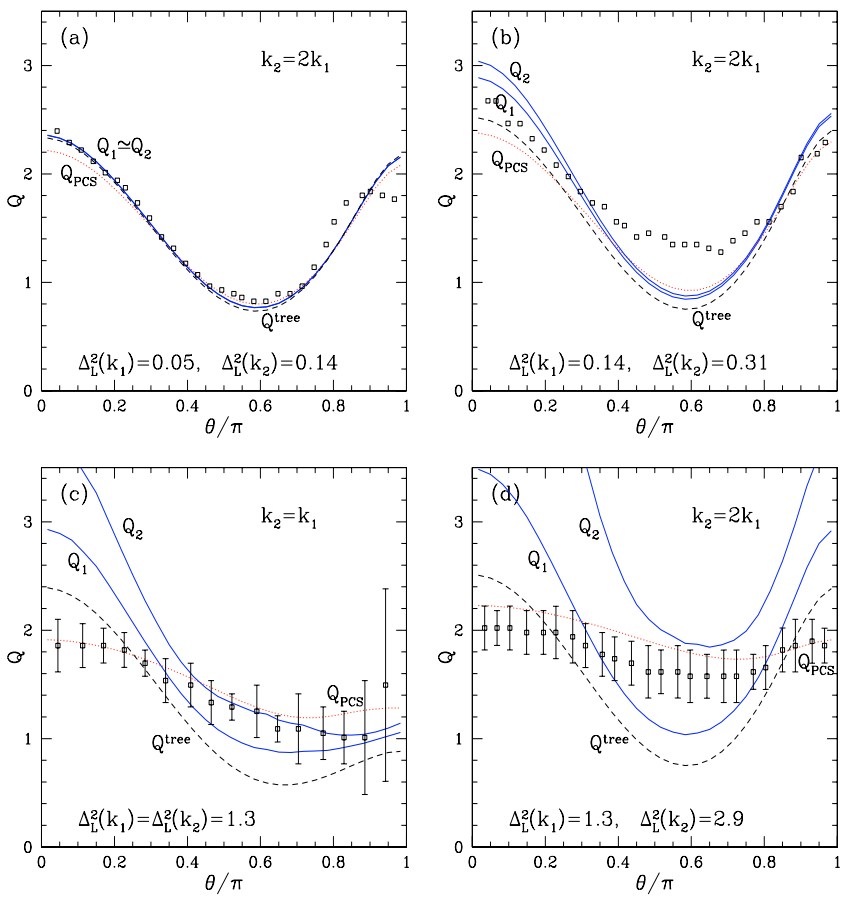

Fig. 17. The reduced bispectrum $Q$ as a function of the angle $\theta$ between wavenumbers $\boldsymbol{k}_{1}$ and $\boldsymbol{k}_{2}$. Panels (a), (b), and (d) have $k_{2}=2 k_{1}$ whereas panel (c) has $k_{2}=k_{1}$. We probe deeper into the nonlinear regime by going from panel (a) to panel (d). The solid line $Q_{1}$ corresponds to the ratio (139) whereas the solid line $Q_{2}$ corresponds to its expansion (140). They are almost identical in panel (a). The dashed line is the tree-order result (143). The dotted line $Q_{\mathrm{PCS}}$ is the phenomenological model of Pan et al. (2007). Data points are taken from Scoccimarro \& Couchman (2001), for panels (a) and (b), and from Fosalba et al. (2005), for panels (c) and (d). They correspond to two different $\Lambda$ CDM cosmologies.

Next, the computation of various diagrams proceeds as in Sect. 7.3. In particular, since the linear growing modes are identical for both dynamics (exponent $\alpha_{1}=1$ ), the time-dependent functions $T_{\left(\ell_{j}\right)}^{(.)}$are identical if all $\ell_{j}$ are equal to 1 . Then, we recover the scalings (118) for the power spectrum and (126), (133), for the bispectrum.

Again, in order to handle the UV divergences associated with diagrams (h) and (i) it is necessary to introduce the regularized contributions $B_{\mathrm{R}}^{(h)}$ and $B_{\mathrm{R}}^{(i)}$, as in Eqs. (135), (136). As discussed in Sect. 7.3, this makes no difference at order $P_{\mathrm{L}}^{3}$ as the UV divergence is associated with higher order terms. On the other hand, for the gravitational dynamics this UV divergence may have a physical meaning. That is, it could be a signature of the breakdown of the equations of motion beyond shell-crossing and may not compensate at higher orders. Indeed, as noticed in Valageas (2002), within the standard perturbative expansion one also encounters UV-divergent diagrams beyond a finite order if $n(\infty)>-3$. Since the large- $N$ expansion has performed a partial resummation of an infinite series of diagrams it is not too surprising to encounter such UV-divergent terms. For the Zeldovich dynamics this is not the case, since we know from the exact nonlinear results that these divergences must eventually compensate once we include all diagrams of a given order, even though the Eulerian description also breaks down beyond shellcrossing. However, for the gravitational dynamics, where there is no such explicit nonlinear solution, it is not known whether these divergences must also fully cancel out (nevertheless they must compensate at least up to the last regular order predicted by the standard expansion scheme). We do not give the explicit expressions of the diagrams obtained in this fashion here as they are too lengthy. They are similar to those obtained within the expansion over $K_{\mathrm{s}}$, given in the Appendix, with the addition of time-dependent functions $T_{\left(\ell_{j}\right)}^{(a, f, g, h, i)}\left(\omega_{j} D\right)$, as described in details in Sect. 7.3 for the simpler case of the Zeldovich dynamics.

Since we use Eqs.(109) and (117) for the nonlinear two-point functions the reduced bispectrum $Q$ defined in Eq. (137) reads as

$Q_{\cos }\left(k_{1}, k_{2}, k_{3}\right)=\frac{B_{\cos }\left(k_{1}, k_{2}, k_{3}\right)}{P_{\mathrm{L}}\left(k_{2}\right) P_{\mathrm{L}}\left(k_{3}\right)+2 \text { perm. }}$,

where the subscript "cos" stands for the steepest-descent method with the original cosine cutoffs of Eqs. (109), (117). At this oneloop order, $B_{\text {cos }}$ is given by the diagrams of Fig. 10 , as discussed above.

As noticed in Sect. 6, it is possible to consider an alternative resummation scheme for the bispectrum, based on the diagrams of Fig. 10 associated with the large- $N$ steepest-descent method, but using the two-point functions (82), (86), given by the large- $k$ partial resummation associated with the random uniform advection of large-scale structures. This amounts to replace the cosine cutoffs of the one-loop steepest-descent predictions (109), (117), by the Gaussian cutoffs of Eqs. (82), (86). Therefore, the structure of the various contributions to the bispectrum $B$ is the same as for the genuine steepest-descent method discussed above. The only change is that the time-dependent functions $T_{\left(\ell_{j}\right)}^{(a, f, g, h, i)}\left(\omega_{j} D\right)$ are different as they involve integrals over Gaussian factors instead of cosines. Nevertheless, the scalings (124) and (134) remain valid,

$D \omega_{j} \gg 1: \quad T^{(\text {tree })} \sim \frac{1}{D \omega}, T^{(1 \text { loop })} \sim \frac{1}{(D \omega)^{3}}$,

where we noted by $T^{\text {(tree) }}$ the time-dependent functions associated with the tree diagram of Fig. 10 and by $T^{(1 \text { loop })}$ the timedependent functions associated with the one-loop diagrams of Fig. 10. Indeed, the scalings (148) simply come from the single and triple integrations over past times associated with the tree and one-loop diagrams. For large $D \omega$, only recent times $D^{\prime}$, with $\left(D-D^{\prime}\right) \lesssim 1 / \omega$, contribute because of the Gaussian or cosine cutoffs. This leads to Eq. (148). Therefore, we again recover the same scalings (126) and (133) for the tree and one-loop contributions to the bispectrum $B$. Then, the reduced bispectrum $Q$ is again given by Eq. (147), but using a subscript "Gauss" to denote the Gaussian cutoffs associated with the resummation of Sect. 6.

Thus, for the equal-time bispectrum the form of the differenttime decay of two-point functions does not really matter since it merely yields the scalings (148). Of course, for the differenttime three-point correlation we recover the decay of the twopoint functions. For instance, we obtain up to one-loop order from the diagrams of Fig. 10

$D_{j} \omega_{j} \gg 1: B \sim \mathrm{e}^{-\frac{1}{2}\left[\left(D_{2}-D_{1}\right)^{2} \omega_{2}^{2}+\left(D_{3}-D_{1}\right)^{2} \omega_{3}^{2}\right]}+2$ perm.

if we use the Gaussian decay.

We show in Fig. 18 the results obtained from the two methods described above for the reduced bispectrum as a function of wavenumber $k$ for equilateral triangles. We can see that the the curves agree on large scales with each other and with the numerical simulations, up to $0.2 \mathrm{~h} \mathrm{Mpc}^{-1}$. The comparison with Figs. 15, 16, shows that the approximations (109), (117), or (82), (86), for the nonlinear two-point functions, are accurate enough 


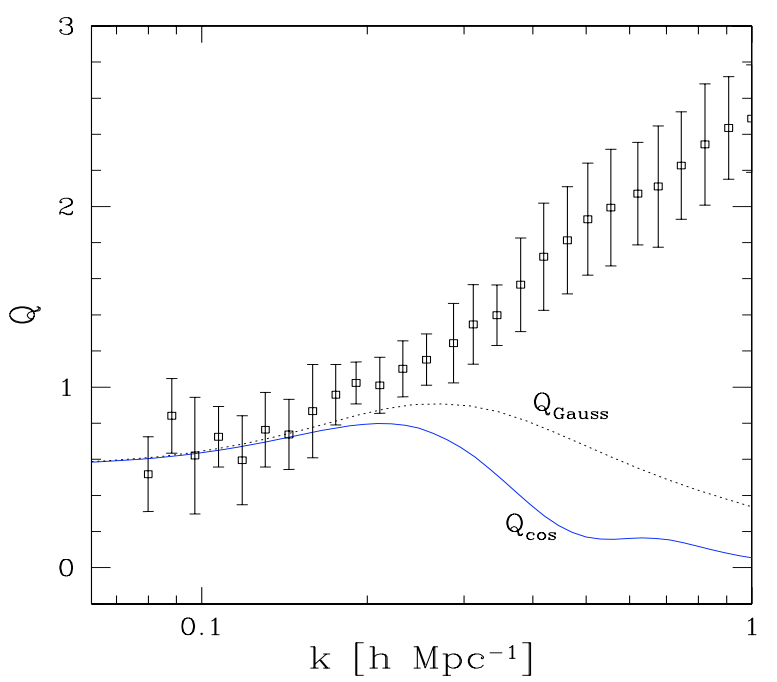

Fig. 18. The reduced bispectrum $Q$ as a function of wavenumber $k$ for equilateral triangles $k_{1}=k_{2}=k_{3}=k$, as in Fig. 15. We show the prediction of the one-loop steepest-descent method from Eq. (147) (solid line $\left.Q_{\text {cos }}\right)$ and the results obtained by using a Gaussian cutoff as in Eqs. (82), (86) (dotted line $Q_{\text {Gauss }}$ ).

to recover the deviations from the constant tree-order result associated with the one-loop order corrections. On small scales, the reduced bispectra $Q_{\mathrm{cos}}$ and $Q_{\text {Gauss }}$ show a slow decay, in agreement with the scalings (118) and (126), (133), which give

$D \omega_{j} \gg 1: \quad Q \sim \frac{1}{D \sigma_{8}}$.

The curve $Q_{\text {cos }}$ displays some oscillations due to the cosine factors whereas the curve $Q_{\text {Gauss }}$ shows a monotonous decay due to the Gaussian factors.

We show in Fig. 19 our results for the bispectrum as a function of the angle $\theta$ between wavenumbers $\boldsymbol{k}_{1}, \boldsymbol{k}_{2}$. We can see that the results are similar to those obtained in Fig. 17 from the expansion over $K_{\mathrm{s}}$ (equivalent to the standard perturbation theory). In particular, they do not manage to reproduce the flattening of the reduced bispectrum as a function of $\theta$ on smaller scales. Therefore, the predictions obtained in this manner from the steepest-descent formalism do not bring a significant improvement over the standard perturbation theory, as shown by the comparison with Figs. 15, 17.

\subsection{Using the nonlinear power spectrum}

We do not compute numerically in this paper the predictions associated with the 2PI effective action method of Sect. 5.2 because there is no simple approximation such as Eqs. (109), (117), or Eqs. (82), (86), for the two-point functions. Moreover, the integrals over time no longer factorize through functions $T_{\left(\ell_{j}\right)}^{(a, f, g, h, i)}\left(\omega_{j} D\right)$, since the internal lines of the one-loop diagrams are nonlinear two-point functions. Thus, even if the latter are approximated by Eqs. (109), (117), or Eqs. (82), (86), one encounters integrals over internal times $D_{j}^{\prime}$ and wavenumbers $q_{j}$ of terms such as $\mathrm{e}^{-\left(D_{j}-D_{j}^{\prime}\right) q_{j}^{2} \sigma_{v}^{2} / 2}$ instead of power-law prefactors $\left(D_{j}^{\prime} / D_{j}\right)^{\alpha}$. All this would make the computation of the 2PI effective action predictions much more complex.

However, to investigate the effect of using more accurate nonlinear two-point functions in all lines of the diagrams of Fig. 10, we modify the approximation $Q_{\text {Gauss }}$ discussed above
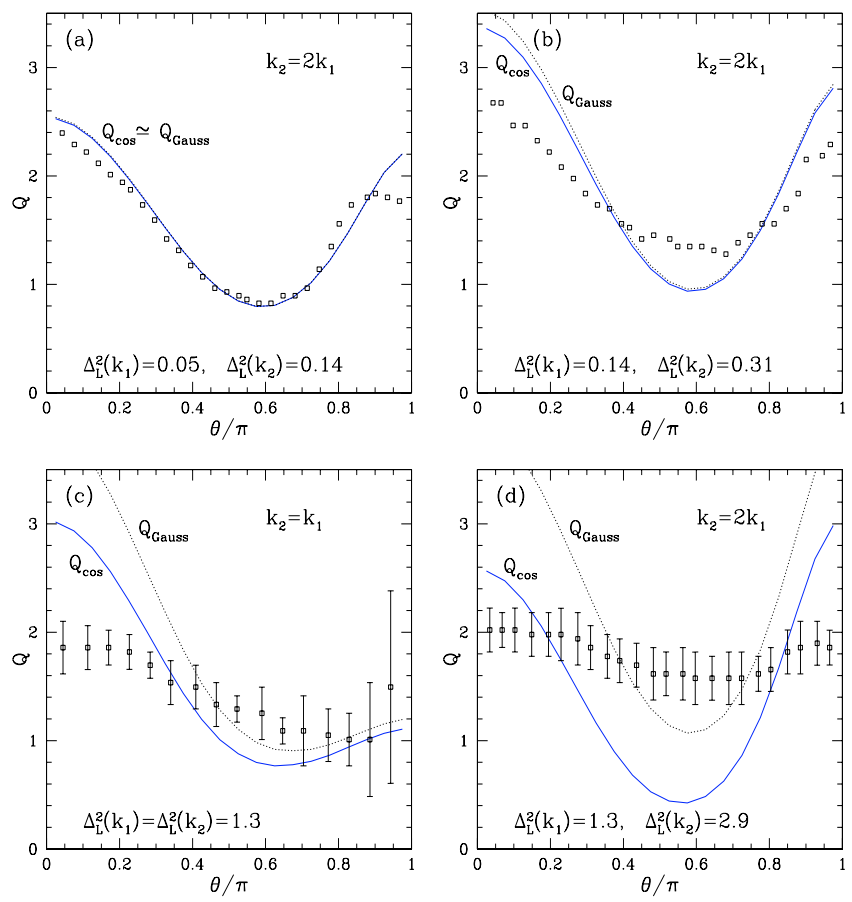

Fig. 19. The reduced bispectrum $Q$ as a function of the angle $\theta$ between wavenumbers $\boldsymbol{k}_{1}$ and $\boldsymbol{k}_{2}$, as in Fig. 19. The curves $Q_{\cos }$ (solid line) and $Q_{\text {Gauss (dotted line) are the predictions obtained from the one- }}$ loop steepest-descent method by using, i) the original nonlinear twopoint functions with a cosine cutoff, ii) the two-point functions with a Gaussian cutoff.

by substituting the fit $P_{\mathrm{NL}}$ of the nonlinear power spectrum, obtained from numerical simulations by Smith et al. (2003), into the diagrams. In other words, we use the exact equal-time nonlinear two-point functions in all lines, and the dependence on the time-difference on external lines is given by the Gaussian factor of Eqs. (82), (86). We keep the power-law time factors in the internal lines so as to keep the factorization through the functions $T_{\left(\ell_{j}\right)}^{(a, f, g, h, i)}\left(\omega_{j} D\right)$. In this fashion, we only need the nonlinear matter power-spectrum given by numerical simulations at the redshift of interest, where we wish to compute the equal-time bispectrum. Then, the equal-time reduced bispectrum $Q$ is computed by using also the fit $P_{\mathrm{NL}}$ of the nonlinear power spectrum (from Smith et al. 2003) in the denominator of Eq. (137). We could apply the same procedure using the cosine time-dependent cutoff of Eqs. (109), (117), however since it does not fare as well as the Gaussian cutoff, we only show in Figs. 20, 21 our results obtained with the Gaussian decay.

In principle, we could improve this procedure in two ways. First, we could use in the external lines the actual two-time density correlation measured in simulations, instead of the Gaussian ansatz. Second, we could also use this in the internal lines. However, we leave this for future works, since the differenttimes density correlation has not been studied in details in current numerical simulations, and this would imply heavier computations for the relevant diagrams as explained above. Nevertheless, note that the different-time Gaussian decay agrees reasonably well with numerical simulations for the response function (Crocce \& Scoccimarro 2007b) and it should also provide a reasonable guess for the nonlinear correlation function as discussed in Valageas (2007b), since it describes the random advection of small-scale structures by the larger-scale coherent flow which affects both two-point functions. 


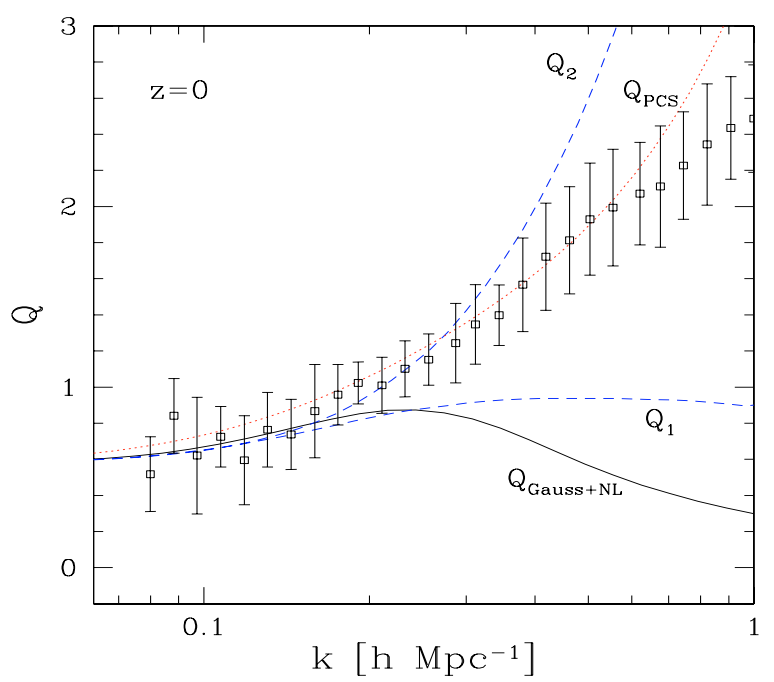

Fig. 20. The reduced bispectrum $Q$ as a function of wavenumber $k$ for equilateral triangles $k_{1}=k_{2}=k_{3}=k$, as in Fig. 18, but using the nonlinear power spectrum from Smith et al. (2003) for the two-point correlations within the one-loop steepest-descent diagrams, together with the Gaussian decay ansatz (solid line $Q_{\text {Gauss+NL }}$ ). We also plot for comparison the standard one-loop results, from the ratio (139) (lower dashed line $Q_{1}$ ), and from its expansion (140) (upper dashed line $Q_{2}$ ), as well as the phenomenological model of Pan et al. (2007) (dotted line $Q_{\mathrm{PCS}}$ ).

We show our results for equilateral triangles as a function of wavenumber $k$ in Fig. 20 (curve $Q_{\text {Gauss }+N L}$ ). For comparison, we also display the predictions of the standard one-loop perturbation theory (curves $Q_{1}$ and $Q_{2}$ associated with Eqs. (139), (140)) and the phenomenological model of Pan et al. (2007). We can see that using the "exact" nonlinear power spectrum $P_{\mathrm{NL}}$ does not change much the results as compared with Fig. 18. The failure to match the numerical simulations beyond $0.2 \mathrm{~h} \mathrm{Mpc}^{-1}$ shows that in order to improve this prediction it is not sufficient to improve the two-point functions alone. One needs to include additional diagrams to those displayed in Fig. 10, such as those obtained by adding new lines that connect the external to the internal lines and which cannot be factorized in the form of Fig. 10 (this requires new two-loop or higher-order diagrams).

The dependence on the angle $\theta$ between wavenumbers $\boldsymbol{k}_{1}$ and $\boldsymbol{k}_{2}$ is displayed in Fig. 21. We only show the standard oneloop prediction $Q_{1}$ for comparison, because it fares better than the alternative $Q_{2}$, see Fig. 17. Here we can see a significant improvement over the previous predictions since we partly recover the weakening of the dependence on $\theta$ in the nonlinear regime. The improvement is most significant in panel (c) (where $\left.\Delta_{L}^{2}(k)=1.3\right)$. At smaller scales the prediction $Q_{\text {Gauss }}$ is too low as compared with the simulations, since it decays as in Eq. (150), as seen in Fig. 20 too. Thus, although it did not make much change for equilateral configurations (Fig. 20), using accurate two-point functions within such frameworks can improve the detailed angular behavior of the bispectrum in the weakly nonlinear regime.

This means that resummation schemes can indeed improve over the standard perturbation theory (as shown by the comparison with the standard one-loop prediction $Q_{1}$ in Fig. 21). However, phenomenological models (such as Pan et al. 2007) can still offer a better match to numerical simulations. This is not completely surprising since the resummation schemes discussed in this paper are still expected to break down at small scales, would it be only for the effect of shell-crossing that
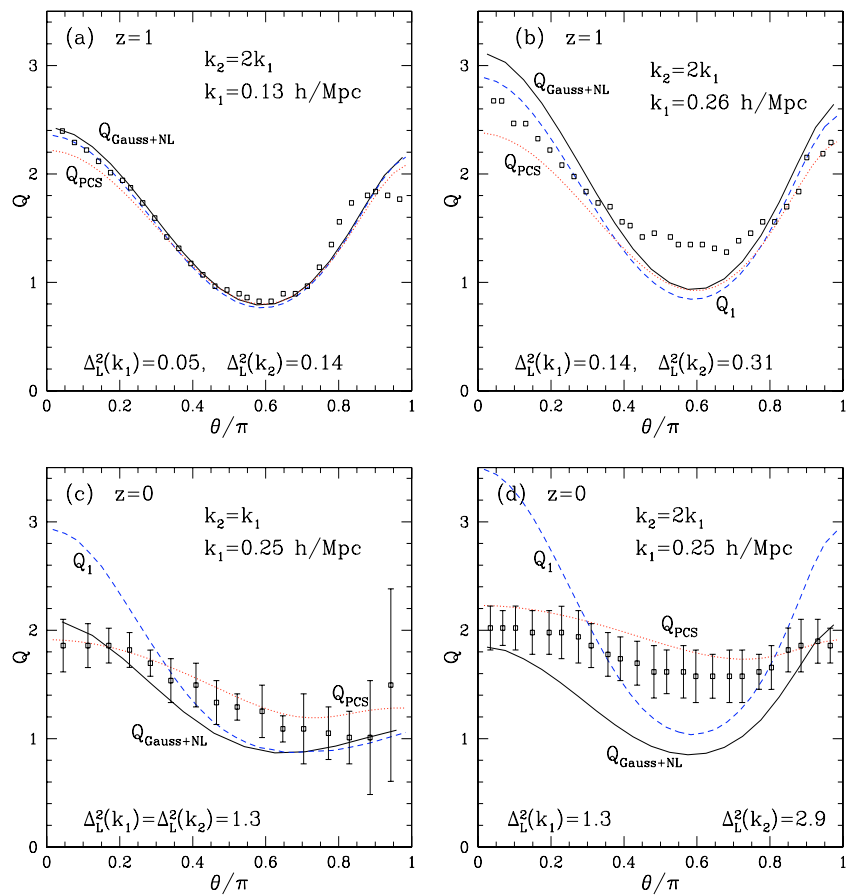

Fig. 21. The reduced bispectrum $Q$ as a function of the angle $\theta$ between wavenumbers $\boldsymbol{k}_{1}$ and $\boldsymbol{k}_{2}$, as in Fig. 19, but using the nonlinear power spectrum from Smith et al. (2003) for the two-point correlations within the one-loop steepest-descent diagrams, together with the Gaussian decay ansatz (solid line $Q_{\text {Gauss+NL }}$ ).

invalidates the equations of motion from which they are derived (although one could apply similar methods to the Vlasov equation, see Valageas 2004). On the other hand, being derived in a systematic fashion from the equations of motion, perturbative methods (that can involve various resummation procedures) are better controlled. Therefore, they should be useful and more reliable than simple models in their regime of validity.

\section{Conclusion}

In this article, we have described several methods to obtain systematic expansions for two-point and three-point correlation functions for the cosmological large-scale density field. These methods also extend to higher-order correlations and response functions (e.g., the response of the two-point and three-point correlations to external perturbations or to the initial conditions). They also apply to related systems, defined by similar hydrodynamical equations, such as the Zeldovich dynamics. We showed how the simplest perturbative expansion derived from the the path-integral formulation of the system (i.e. expanding over the cubic part of the action) corresponds to the equivalent standard expansion directly obtained from the hydrodynamical equations of motion. Next, we described two resummations, associated with "large- $N$ " limits, that can be conveniently derived from the path integral (Valageas 2007a,b), and a high- $k$ resummation that is most easily derived from the equation of motion (Crocce \& Scoccimarro 2006a,b; Valageas 2007b). Thus, we showed how to derive expansions for high-order correlations (or responses) and we compared the resummations involved in these various schemes.

We first applied these methods up to one-loop order to the simpler case of the Zeldovich dynamics, where explicit expressions are simple enough to be given here. We explicitly checked 
that the perturbative expansion of the path integral recovers the standard perturbative expansion over powers of the linear density field. However, since these two expansions are organized in different manners, we found that two diagrams, obtained within the former method for the three-point correlation, exhibit an additional UV divergence (for $n(\infty)>-3$ ). This divergence cancels out as we sum over all diagrams but it shows explicitly that some care must be taken in using these expansions. For numerical purposes, it is best to first regularize each diagram by subtracting the divergent parts that cancel out. Next, we applied the large- $N$ steepest-descent method to this Zeldovich dynamics. We showed how the time-dependence can be factorized, through functions $T\left(\omega_{j} D\right)$, that measure the decay of different-time correlation and response functions in the nonlinear regime (within simple approximations). We found that, contrary to the standard expansions, tree diagrams and one-loop diagrams obey the same scaling laws over the linear amplitude $D \sigma_{8}$. This is due to the cutoff $T\left(\omega_{j} D\right)$ associated with the partial resummations. We also explained how it is again possible to regularize the expansion, as for the previous expansion.

Second, we applied these expansion schemes to the gravitational dynamics. All properties obtained for the Zeldovich dynamics remain valid and we have compared our results with numerical simulations. We checked that on quasi-linear scales all expansion schemes agree with one another and with the numerical simulations, up to $0.2 \mathrm{~h} \mathrm{Mpc}^{-1}$ (where $\Delta_{L}^{2}(k) \simeq 1$ for a $\Lambda \mathrm{CDM}$ power spectrum with $\sigma_{8}=0.9$ ). At smaller scales they deviate from one another and from the numerical simulations. We recovered the known failure of the standard perturbative expansion to reproduce the observed flattening in the nonlinear regime of the reduced bispectrum $Q\left(k_{1}, k_{2}, k_{3}\right)$ as a function of the angle $\theta$ between wavenumbers $\boldsymbol{k}_{1}$ and $\boldsymbol{k}_{2}$. We found that the steepest-descent resummation does not fare much better. Even substituting the Gaussian decay obtained in a high- $k$ limit for the different-time two-point functions does not help (it actually increases somewhat further the variation with $\theta$ ). Nevertheless, the agreement with numerical simulations significantly improves if we use the "exact" nonlinear power spectrum (obtained from a fit to simulations) for the two-point correlation within this formalism (together with the Gaussian decay). This does not extend the range of validity for equilateral configurations towards higher $k$, but it manages to partly recover the observed flattening of the reduced bispectrum as a function of $\theta$.

We have also compared the predictions obtained from these expansion schemes with the simple phenomenological model of Pan et al. (2007). It appears that the validity of the latter extends to smaller scales, up to $0.8 \mathrm{~h} \mathrm{Mpc}^{-1}$ where $\Delta_{L}^{2}(k) \simeq 17$ in the case studied here. Since this model is also much simpler to use, it seems that the scale transformations devised by Hamilton et al. (1991) may indeed provide remarkably efficient tools to investigate various statistics associated with gravitational clustering. At quasi-linear scales $k \sim 0.1 h \mathrm{Mpc}^{-1}$, the systematic expansion schemes studied in this article may be somewhat more accurate (since they arise from rigorous systematic expansions) whereas the phenomenological model of Pan et al. (2007) may lead to a slight overestimate of the bispectrum (which would not be surprising since its expansion is not expected to agree with perturbation theory).

The results obtained in this article suggest that, in order to make further progress along these lines, one needs to include higher-order diagrams (two-loop diagrams or higher) to obtain reliable predictions for the bispectrum that accurately reproduce the weakening of the dependence on the angle $\theta$ in the nonlinear regime. Moreover, using accurate two-point functions provides a significant improvement. This requires both the use of a Gaussian decay for different-time quantities (or a similar cutoff that agrees with numerical simulations) and of a reasonable equal-time correlation (e.g. using the nonlinear power spectrum measured in simulations). On the other hand, the path-integral formulation might serve as a basis for other approximation methods than the expansion schemes discussed here.

Alternative methods, based on phenomenological models, such as the halo model (Cooray \& Sheth 2002) or the scaling transformation of Hamilton et al. (1991), may provide other means to describe the statistical properties of gravitational clustering. Although simpler and more efficient in the nonlinear regime, they do not have the rigorous and systematic character of expansion schemes. Moreover, they often involve several free parameters that must be fitted to simulations and they are not derived from first principles. It would be interesting to develop a connection between such phenomenological approaches and more systematic methods as those developed in this article. However, it is not clear whether such a connection can be achieved at a detailed level. This issue is left for future works.

\section{Appendix A: Bispectrum for the gravitational dynamics: tree and one-loop diagrams for the expansion over $K_{s}$}

We give in this Appendix the expressions of the diagrams of Fig. 6, associated with the expansion over powers of $K_{\mathrm{s}}$ of the path integral defining the bispectrum, for the case of the gravitational dynamics. The corresponding results for the Zeldovich dynamics are given in Sect. 7.2. The expressions below are similar to the former but the rational functions which appear in the integrands are more intricate. First, the tree-diagram (a) is:

$$
\begin{aligned}
B^{(a)}\left(k_{1}, k_{2}, k_{3}\right)= & P_{\mathrm{L}}\left(k_{2}\right) P_{\mathrm{L}}\left(k_{3}\right)\left[\frac{10}{7}+\left(\frac{k_{2}}{k_{3}}+\frac{k_{3}}{k_{2}}\right) \frac{\boldsymbol{k}_{2} \cdot \boldsymbol{k}_{3}}{k_{2} k_{3}}\right. \\
& \left.+\frac{4\left(\boldsymbol{k}_{2} \cdot \boldsymbol{k}_{3}\right)^{2}}{7 k_{2}^{2} k_{3}^{2}}\right]+2 \text { perm. }
\end{aligned}
$$

The diagram (f) which involves a triple integration over the linear power spectrum reads as

$$
\begin{aligned}
B^{(f)}= & \int \mathrm{d} \boldsymbol{q}_{1} \mathrm{~d} \boldsymbol{q}_{2} \mathrm{~d} \boldsymbol{q}_{3} \delta_{\mathrm{D}}\left(\boldsymbol{q}_{1}+\boldsymbol{q}_{2}-\boldsymbol{k}_{1}\right) \\
& \times \delta_{\mathrm{D}}\left(\boldsymbol{q}_{1}+\boldsymbol{q}_{3}+\boldsymbol{k}_{2}\right) P_{\mathrm{L}}\left(q_{1}\right) P_{\mathrm{L}}\left(q_{2}\right) P_{\mathrm{L}}\left(q_{3}\right) \\
& \times \frac{10 q_{1}^{2} q_{2}^{2}+7\left(q_{1}^{2}+q_{2}^{2}\right)\left(\boldsymbol{q}_{1} \cdot \boldsymbol{q}_{2}\right)+4\left(\boldsymbol{q}_{1} \cdot \boldsymbol{q}_{2}\right)^{2}}{7 q_{1}^{2} q_{2}^{2}} \\
& \times \frac{10 q_{1}^{2} q_{3}^{2}+7\left(q_{1}^{2}+q_{3}^{2}\right)\left(\boldsymbol{q}_{1} \cdot \boldsymbol{q}_{3}\right)+4\left(\boldsymbol{q}_{1} \cdot \boldsymbol{q}_{3}\right)^{2}}{7 q_{1}^{2} q_{3}^{2}} \\
& \times \frac{10 q_{2}^{2} q_{3}^{2}-7\left(q_{2}^{2}+q_{3}^{2}\right)\left(\boldsymbol{q}_{2} \cdot \boldsymbol{q}_{3}\right)+4\left(\boldsymbol{q}_{2} \cdot \boldsymbol{q}_{3}\right)^{2}}{7 q_{2}^{2} q_{3}^{2}}
\end{aligned}
$$

Next, the two diagrams (b) and (g) which involve a double 
integration over $P_{\mathrm{L}}$ are:

$$
\begin{aligned}
B^{(b)}= & -P_{\mathrm{L}}\left(k_{1}\right) \int \mathrm{d} \boldsymbol{q}_{1} \mathrm{~d} \boldsymbol{q}_{2} \delta_{\mathrm{D}}\left(\boldsymbol{q}_{1}+\boldsymbol{q}_{2}-\boldsymbol{k}_{2}\right) \\
& \times \frac{10 q_{1}^{2} q_{2}^{2}+7\left(q_{1}^{2}+q_{2}^{2}\right)\left(\boldsymbol{q}_{1} \cdot \boldsymbol{q}_{2}\right)+4\left(\boldsymbol{q}_{1} \cdot \boldsymbol{q}_{2}\right)^{2}}{7 q_{1}^{2} q_{2}^{2}} \\
& \times\left\{\frac{7 k_{2}^{2}\left(\boldsymbol{k}_{1} \cdot \boldsymbol{k}_{3}\right)\left[10 q_{1}^{2} q_{2}^{2}+7\left(q_{1}^{2}+q_{2}^{2}\right)\left(\boldsymbol{q}_{1} \cdot \boldsymbol{q}_{2}\right)+4\left(\boldsymbol{q}_{1} \cdot \boldsymbol{q}_{2}\right)^{2}\right]}{252 k_{1}^{2} k_{2}^{2} q_{1}^{2} q_{2}^{2}}\right. \\
& +\frac{7 k_{1}^{2}\left(\boldsymbol{k}_{2} \cdot \boldsymbol{k}_{3}\right)-2 k_{3}^{2}\left(\boldsymbol{k}_{1} \cdot \boldsymbol{k}_{2}\right)}{252 k_{1}^{2} k_{2}^{2}}\left[6+\frac{7\left(q_{1}^{2}+q_{2}^{2}\right)\left(\boldsymbol{q}_{1} \cdot \boldsymbol{q}_{2}\right)}{q_{1}^{2} q_{2}^{2}}\right. \\
& \left.\left.+\frac{8\left(\boldsymbol{q}_{1} \cdot \boldsymbol{q}_{2}\right)^{2}}{q_{1}^{2} q_{2}^{2}}\right]\right\} P_{\mathrm{L}}\left(q_{1}\right) P_{\mathrm{L}}\left(q_{2}\right)+5 \text { perm. },
\end{aligned}
$$

$$
B^{(g)}=P_{\mathrm{L}}\left(k_{1}\right) \int \mathrm{d} \boldsymbol{q}_{1} \mathrm{~d} \boldsymbol{q}_{2} \delta_{\mathrm{D}}\left(\boldsymbol{q}_{1}+\boldsymbol{q}_{2}-\boldsymbol{k}_{2}\right)
$$$$
\times P_{\mathrm{L}}\left(q_{1}\right) P_{\mathrm{L}}\left(q_{2}\right) \frac{10\left(\boldsymbol{k}_{2} \cdot \boldsymbol{q}_{1}\right)\left(\boldsymbol{k}_{2} \cdot \boldsymbol{q}_{2}\right)-3 k_{2}^{2}\left(\boldsymbol{q}_{1} \cdot \boldsymbol{q}_{2}\right)}{q_{1}^{2} q_{2}^{2}}
$$$$
\times\left\{\frac{2\left(\boldsymbol{k}_{1} \cdot \boldsymbol{q} 1\right)\left[10 k_{3}^{2} q_{2}^{2}+\left(7 q_{2}^{2}-11 k_{3}^{2}-14\left(\boldsymbol{k}_{3} \cdot \boldsymbol{q}_{2}\right)\right)\left(\boldsymbol{k}_{3} \cdot \boldsymbol{q}_{2}\right)\right]}{882 k_{1}^{2} q_{1}^{2} q_{2}^{2}}\right.
$$$$
+\frac{\left(\boldsymbol{k}_{1} \cdot \boldsymbol{k}_{3}\right)\left[\left(14 q_{2}^{2}+41 k_{3}^{2}+70\left(\boldsymbol{k}_{3} \cdot \boldsymbol{q}_{2}\right)\right)\left(\boldsymbol{k}_{3} \cdot \boldsymbol{q}_{2}\right)-15 k_{3}^{2} q_{2}^{2}\right]}{882 k_{1}^{2} q_{2}^{2}\left|\boldsymbol{k}_{3}+\boldsymbol{q}_{2}\right|^{2}}
$$$$
-\frac{q_{1}^{2}\left(\boldsymbol{k}_{1} \cdot \boldsymbol{q}_{2}\right)+k_{1}^{2}\left(\boldsymbol{q}_{1} \cdot\left(\boldsymbol{k}_{3}+\boldsymbol{q}_{2}\right)\right)}{882 k_{1}^{2} q_{1}^{2}}\left[\frac{15 k_{3}^{2}}{\left|\boldsymbol{k}_{3}+\boldsymbol{q}_{2}\right|^{2}}\right.
$$$$
\left.\left.-\frac{\left(\boldsymbol{k}_{3} \cdot \boldsymbol{q}_{2}\right)\left(14 q_{2}^{2}+41 k_{3}^{2}+70\left(\boldsymbol{k}_{3} \cdot \boldsymbol{q}_{2}\right)\right)}{q_{2}^{2}\left|\boldsymbol{k}_{3}+\boldsymbol{q}_{2}\right|^{2}}\right]\right\}+5 \text { perm. }
$$

Finally, the five diagrams (c), (d), (e), (h), and (i), which involve a single integration over $P_{\mathrm{L}}$, are given by:

$$
\begin{aligned}
B^{(c)} & =P_{\mathrm{L}}\left(k_{2}\right) P_{\mathrm{L}}\left(k_{3}\right) \frac{10 k_{2}^{2} k_{3}^{2}+7\left(k_{2}^{2}+k_{3}^{2}\right)\left(\boldsymbol{k}_{2} \cdot \boldsymbol{k}_{3}\right)+4\left(\boldsymbol{k}_{2} \cdot \boldsymbol{k}_{3}\right)^{2}}{7 k_{2}^{2} k_{3}^{2}} \\
& \times \int \mathrm{d} \boldsymbol{q} P_{\mathrm{L}}(q)\left[\frac{30 k_{2}^{2} q^{2}+\left(47 k_{2}^{2}-7 q^{2}\right)\left(\boldsymbol{k}_{2} \cdot \boldsymbol{q}\right)}{126 q^{2}\left|\boldsymbol{k}_{2}-\boldsymbol{q}\right|^{2}}\right. \\
& +\frac{-\left(63 k_{2}^{4}+163 k_{2}^{2} q^{2}\right)\left(\boldsymbol{k}_{2} \cdot \boldsymbol{q}\right)^{2}+2\left(71 k_{2}^{2}+35 q^{2}\right)\left(\boldsymbol{k}_{2} \cdot \boldsymbol{q}\right)^{3}}{126 k_{2}^{2} q^{4}\left|\boldsymbol{k}_{2}-\boldsymbol{q}\right|^{2}} \\
& \left.-\frac{56\left(\boldsymbol{k}_{2} \cdot \boldsymbol{q}\right)^{4}}{126 k_{2}^{2} q^{4}\left|\boldsymbol{k}_{2}-\boldsymbol{q}\right|^{2}}\right]+5 \text { perm. }
\end{aligned}
$$

$$
\begin{aligned}
& B^{(d)}=P_{\mathrm{L}}\left(k_{2}\right) P_{\mathrm{L}}\left(k_{3}\right) \int \mathrm{d} \boldsymbol{q} P_{\mathrm{L}}(q)\left\{\frac{9 k_{3}^{2}\left(\boldsymbol{k}_{1} \cdot \boldsymbol{k}_{2}\right)-2 k_{1}^{2}\left(\boldsymbol{k}_{2} \cdot \boldsymbol{k}_{3}\right)}{k_{2}^{2} k_{3}^{2}}\right. \\
& \times\left[\frac{2\left(\boldsymbol{k}_{2} \cdot \boldsymbol{q}\right)^{3}\left(4\left(\boldsymbol{k}_{2} \cdot \boldsymbol{q}\right)-5 q^{2}\right)+k_{2}^{4}\left(6 q^{4}-29 q^{2}\left(\boldsymbol{k}_{2} \cdot \boldsymbol{q}\right)+21\left(\boldsymbol{k}_{2} \cdot \boldsymbol{q}\right)^{2}\right)}{1386 k_{2}^{2} q^{4}\left|\boldsymbol{k}_{2}-\boldsymbol{q}\right|^{2}}\right. \\
& \left.+\frac{k_{2}^{2}\left(\boldsymbol{k}_{2} \cdot \boldsymbol{q}\right)\left(-11 q^{4}+49 q^{2}\left(\boldsymbol{k}_{2} \cdot \boldsymbol{q}\right)-34\left(\boldsymbol{k}_{2} \cdot \boldsymbol{q}\right)^{2}\right)}{1386 k_{2}^{2} q^{4}\left|\boldsymbol{k}_{2}-\boldsymbol{q}\right|^{2}}\right] \\
& +\frac{3\left(\boldsymbol{k}_{1} \cdot \boldsymbol{k}_{3}\right)\left[-\frac{k_{2}^{2}\left(30 q^{4}+47 q^{2}\left(\boldsymbol{k}_{2} \cdot \boldsymbol{q}\right)-63\left(\boldsymbol{k}_{2} \cdot \boldsymbol{q}\right)^{2}\right)}{1386 q^{4}\left|\boldsymbol{k}_{2}-\boldsymbol{q}\right|^{2}}\right.}{k_{3}^{2}}\left[-\frac{\left(\boldsymbol{k}_{2} \cdot \boldsymbol{q}\right)\left(7 q^{4}+163 q^{2}\left(\boldsymbol{k}_{2} \cdot \boldsymbol{q}\right)-142\left(\boldsymbol{k}_{2} \cdot \boldsymbol{q}\right)^{2}\right)}{1386 q^{4}\left|\boldsymbol{k}_{2}-\boldsymbol{q}\right|^{2}}\right. \\
& \left.\left.\quad+\frac{14\left(\boldsymbol{k}_{2} \cdot \boldsymbol{q}\right)^{3}\left(4\left(\boldsymbol{k}_{2} \cdot \boldsymbol{q}\right)-5 q^{2}\right)}{1386 k_{2}^{2} q^{4}\left|\boldsymbol{k}_{2}-\boldsymbol{q}\right|^{2}}\right]\right\}+5 \text { perm. },
\end{aligned}
$$

$$
\begin{gathered}
B^{(e)}=-P_{\mathrm{L}}\left(k_{2}\right) P_{\mathrm{L}}\left(k_{3}\right) \int \mathrm{d} \boldsymbol{q} P_{\mathrm{L}}(q)\left\{\frac{k_{3}^{2}\left(\boldsymbol{k}_{1} \cdot \boldsymbol{k}_{2}\right)+k_{2}^{2}\left(\boldsymbol{k}_{1} \cdot \boldsymbol{k}_{3}\right)}{k_{2}^{2} k_{3}^{2}}\right. \\
\times\left[\frac{56 k_{1}^{2} q^{4}+\left(107 k_{1}^{2} q^{2}+33 q^{4}\right)\left(\boldsymbol{k}_{1} \cdot \boldsymbol{q}\right)-5\left(29 k_{1}^{2}+75 q^{2}\right)\left(\boldsymbol{k}_{1} \cdot \boldsymbol{q}\right)^{2}}{1386 q^{4}\left|\boldsymbol{k}_{1}-\boldsymbol{q}\right|^{2}}\right. \\
\left.\quad+\frac{18\left(17 k_{1}^{2}+5 q^{2}\right)\left(\boldsymbol{k}_{1} \cdot \boldsymbol{q}\right)^{3}-72\left(\boldsymbol{k}_{1} \cdot \boldsymbol{q}\right)^{4}}{1386 k_{1}^{2} q^{4}\left|\boldsymbol{k}_{1}-\boldsymbol{q}\right|^{2}}\right]-\frac{2\left(\boldsymbol{k}_{2} \cdot \boldsymbol{k}_{3}\right)}{k_{2} k_{3}} \\
\times\left[\frac{21 k_{1}^{4} q^{4}+\left(34 k_{1}^{4} q^{2}-6 k_{1}^{2} q^{4}\right)\left(\boldsymbol{k}_{1} \cdot \boldsymbol{q}\right)-\left(43 k_{1}^{4}+124 k_{1}^{2} q^{2}\right)\left(\boldsymbol{k}_{1} \cdot \boldsymbol{q}\right)^{2}}{1386 k_{2} k_{3} q^{4}\left|\boldsymbol{k}_{1}-\boldsymbol{q}\right|^{2}}\right. \\
\left.\left.\quad+\frac{2\left(53 k_{1}^{2}+30 q^{2}\right)\left(\boldsymbol{k}_{1} \cdot \boldsymbol{q}\right)^{3}-48\left(\boldsymbol{k}_{1} \cdot \boldsymbol{q}\right)^{4}}{1386 k_{2} k_{3} q^{4}\left|\boldsymbol{k}_{1}-\boldsymbol{q}\right|^{2}}\right]\right\}+2 \text { perm. }
\end{gathered}
$$

$$
\begin{aligned}
& B^{(h)}=-P_{\mathrm{L}}\left(k_{2}\right) P_{\mathrm{L}}\left(k_{3}\right) \int \mathrm{d} \boldsymbol{q} P_{\mathrm{L}}(q)\left\{\frac{3 \boldsymbol{k}_{1} \cdot\left(\boldsymbol{k}_{3}+\boldsymbol{q}\right)}{539\left|\boldsymbol{k}_{3}+\boldsymbol{q}\right|^{2}}\right. \\
& \times \frac{10 k_{2}^{2} q^{2}-7\left(k_{2}^{2}+q^{2}\right)\left(\boldsymbol{k}_{2} \cdot \boldsymbol{q}\right)+4\left(\boldsymbol{k}_{2} \cdot \boldsymbol{q}\right)^{2}}{k_{2}^{2} q^{2}} \\
& \times \frac{6 k_{3}^{2} q^{2}+7\left(k_{3}^{2}+q^{2}\right)\left(\boldsymbol{k}_{3} \cdot \boldsymbol{q}\right)+8\left(\boldsymbol{k}_{3} \cdot \boldsymbol{q}\right)^{2}}{k_{3}^{2} q^{2}} \\
& +\frac{k_{1}^{2}\left[\boldsymbol{q} \cdot\left(-\boldsymbol{k}_{2}+\boldsymbol{k}_{3}+\boldsymbol{q}\right)-\left(\boldsymbol{k}_{2} \cdot \boldsymbol{k}_{3}\right)\right]}{1617 k_{2}^{2} k_{3}^{2}} \\
& \times \frac{6 k_{2}^{2} q^{2}-7\left(k_{2}^{2}+q^{2}\right)\left(\boldsymbol{k}_{2} \cdot \boldsymbol{q}\right)+8\left(\boldsymbol{k}_{2} \cdot \boldsymbol{q}\right)^{2}}{q^{2}\left|\boldsymbol{k}_{2}-\boldsymbol{q}\right|^{2}} \\
& \left.\quad \times \frac{6 k_{3}^{2} q^{2}+7\left(k_{3}^{2}+q^{2}\right)\left(\boldsymbol{k}_{3} \cdot \boldsymbol{q}\right)+8\left(\boldsymbol{k}_{3} \cdot \boldsymbol{q}\right)^{2}}{q^{2}\left|\boldsymbol{k}_{3}+\boldsymbol{q}\right|^{2}}\right\}+5 \text { perm. },
\end{aligned}
$$

$$
\begin{aligned}
B^{(i)} & =P_{\mathrm{L}}\left(k_{2}\right) P_{\mathrm{L}}\left(k_{3}\right) \int \mathrm{d} \boldsymbol{q} P_{\mathrm{L}}(q)\left\{\frac { \boldsymbol { k } _ { 1 } \cdot \boldsymbol { q } } { q ^ { 2 } } \left[\frac{2 k_{2}^{2} q^{2}+\left(k_{2}^{2}+q^{2}\right)\left(\boldsymbol{k}_{2} \cdot \boldsymbol{q}\right)}{k_{2}^{2} q^{2}}\right.\right. \\
& \times\left(\frac{2 \boldsymbol{k}_{3} \cdot\left(\boldsymbol{k}_{2}+\boldsymbol{q}\right)\left|\boldsymbol{k}_{1}-\boldsymbol{q}\right|^{2}-7 k_{3}^{2}\left[\boldsymbol{k}_{1} \cdot \boldsymbol{k}_{2}+\boldsymbol{q} \cdot\left(\boldsymbol{k}_{1}-\boldsymbol{k}_{2}-\boldsymbol{q}\right)\right]}{154 k_{3}^{2}\left|\boldsymbol{k}_{2}+\boldsymbol{q}\right|^{2}}\right. \\
& \left.-\frac{5 \boldsymbol{k}_{3} \cdot\left(\boldsymbol{k}_{1}-\boldsymbol{q}\right)}{66 k_{3}^{2}}\right)+\frac{2 \boldsymbol{k}_{2} \cdot \boldsymbol{q}}{k_{2}^{2}}\left(-\frac{\boldsymbol{k}_{3} \cdot\left(\boldsymbol{k}_{1}-\boldsymbol{q}\right)\left|\boldsymbol{k}_{2}+\boldsymbol{q}\right|^{2}}{66 k_{3}^{2} q^{2}}\right. \\
& \left.\left.+\frac{2 \boldsymbol{k}_{3} \cdot\left(\boldsymbol{k}_{2}+\boldsymbol{q}\right)\left|\boldsymbol{k}_{1}-\boldsymbol{q}\right|^{2}-7 k_{3}^{2}\left[\boldsymbol{k}_{1} \cdot \boldsymbol{k}_{2}+\boldsymbol{q} \cdot\left(\boldsymbol{k}_{1}-\boldsymbol{k}_{2}-\boldsymbol{q}\right)\right]}{231 k_{3}^{2} q^{2}}\right)\right] \\
& +\frac{7 k_{1}^{2} q^{2}+\left(2 k_{1}^{2}-9 q^{2}\right)\left(\boldsymbol{k}_{1} \cdot \boldsymbol{q}\right)}{q^{2}\left|\boldsymbol{k}_{1}-\boldsymbol{q}\right|^{2}}\left[\frac{2 k_{2}^{2} q^{2}+\left(k_{2}^{2}+q^{2}\right)\left(\boldsymbol{k}_{2} \cdot \boldsymbol{q}\right)}{k_{2}^{2} q^{2}}\right. \\
& +\frac{2 \boldsymbol{k}_{3} \cdot\left(\boldsymbol{k}_{2}+\boldsymbol{q}\right)\left|\boldsymbol{k}_{1}-\boldsymbol{q}\right|^{2}-k_{3}^{2}\left[\boldsymbol{k}_{1} \cdot \boldsymbol{k}_{2}+\boldsymbol{q} \cdot\left(\boldsymbol{k}_{1}-\boldsymbol{k}_{2}-\boldsymbol{q}\right)\right]}{462 k_{3}^{2}\left|\boldsymbol{k}_{2}+\boldsymbol{q}\right|^{2}} \\
& \left.-\frac{5 \boldsymbol{k}_{3} \cdot\left(\boldsymbol{k}_{1}-\boldsymbol{q}\right)}{1386 k_{3}^{2}}\right)+\frac{2 \boldsymbol{k}_{2} \cdot \boldsymbol{q}}{k_{2}^{2}}\left(-\frac{\boldsymbol{k}_{3} \cdot\left(\boldsymbol{k}_{1}-\boldsymbol{q}\right)\left|\boldsymbol{k}_{2}+\boldsymbol{q}\right|^{2}}{1386 k_{3}^{2} q^{2}}\right. \\
& \left.\left.\left.+\frac{2 \boldsymbol{k}_{3} \cdot\left(\boldsymbol{k}_{2}+\boldsymbol{q}\right)\left|\boldsymbol{k}_{1}-\boldsymbol{q}\right|^{2}-k_{3}^{2}\left[\boldsymbol{k}_{1} \cdot \boldsymbol{k}_{2}+\boldsymbol{q} \cdot\left(\boldsymbol{k}_{1}-\boldsymbol{k}_{2}-\boldsymbol{q}\right)\right]}{693 k_{3}^{2} q^{2}}\right)\right]\right\} \\
+ & 5 \text { perm. }
\end{aligned}
$$

Because of the property $\boldsymbol{k}_{1}+\boldsymbol{k}_{2}+\boldsymbol{k}_{3}=0$, which has been used to simplify somewhat these expressions, it is possible to write each contribution under many different forms (for instance by replacing $\boldsymbol{k}_{1}$ by $\left.-\left(\boldsymbol{k}_{2}+\boldsymbol{k}_{3}\right)\right)$.

We do not give in this paper the expressions obtained within the steepest-descent expansion, as they are longer than Eqs. (A.1)-(A.9). However, as explained in the main text, by 
putting all time-dependent functions $T_{\left(\ell_{j}\right)}^{(.)}$equal to unity, they reduce to the expressions above for diagrams (a), (f), (g), (h), and (i).

\section{References}

Berges, J. 2002, Nucl. Phys. A, 699, 847

Bernardeau, F., Colombi, S., Gaztanaga, E., \& Scoccimarro, R. 2002, Phys. Rep., 367,1

Bouchaud, J.-P., Cugliandolo, L., Kurchan, J., \& Mezard, M. 1996, Physica A, 226, 243

Cole, S., Percival, W. J., Peacock, J. A., et al. 2005, MNRAS, 362, 505

Cooray, A., \& Sheth, R. 2002, Phys. Rep., 372, 1

Crocce, M., Scoccimarro, R. 2006a, Phys. Rev. D, 73, 063519

Crocce, M., \& Scoccimarro, R. 2006b, Phys. Rev. D, 73, 063520

Crocce, M., \& Scoccimarro, R. 2007 [arXiv: 0704 . 2783]

Eisenstein, D. J., Hu, W., \& Tegmark, M. 1998, ApJ, 504, L57

Eisenstein, D. J., Zehavi, I., Hogg, D. W., et al. 2005, ApJ, 633, 560

Fosalba, P., Pan, J., \& Szapudi, I. 2005, ApJ, 632, 29

Fry, J. N. 1984, ApJ, 279, 499

Goroff, M. H., Grinstein, B., Rey, S.-J., \& Wise, M. B. 1986, ApJ, 311, 6

Grinstein, B., \& Wise, M. B. 1987, ApJ, 320, 448

Gurbatov, S. N., Saichev, A. I., \& Shandarin, S. F. 1989, MNRAS, 236, 385

Hamilton, A. J. S., Kumar, P., Lu, E., \& Matthews, A. 1991, ApJ, 374, L1

Jain, B., \& Bertschinger, E. 1996, ApJ, 456, 43

Kraichnan, R. 1961, J. Fluid Mech., 7, 124

L'vov, V., \& Procaccia, I. 1995, Proceedings of Les Houches summer school [arXiv: chao-dyn/9502010]
McDonald, P. 2007, Phys. Rev. D, 75, 043514

Massey, R., Rhodes, J., Leauthaud, A., et al. 2007, ApJS, 172, 239

Matarrese, S., \& Pietroni, M. 2007a [arXiv:astro-ph/0702653]

Matarrese, S., \& Pietroni, M. 2007b [arXiv: astro-ph/0703563]

Munshi, D., Valageas, P., Van Waerbeke, L., \& Heavens, A. 2007 [arXiv:astro-ph/0612667]

Pan, J., Coles, P., \& Szapudi, I. 2007, submitted [arXiv: astro-ph/0707.1594]

Peebles, P. J. E. 1980, The large scale structure of the universe (Princeton University Press)

Schneider, P., \& Bartelmann, M. 1995, MNRAS, 273, 475

Scoccimarro, R. 1997, ApJ, 487, 1

Scoccimarro, R., \& Couchman, H. M. P. 2001, MNRAS, 325, 1312

Scoccimarro, R., \& Frieman, J. 1996a, ApJS, 105, 37

Scoccimarro, R., \& Frieman, J. 1996b, ApJ, 473, 620

Sefusatti, E., Crocce, M., Pueblas, S., \& Scoccimarro, R. 2006, Phys. Rev. D, 74, 023522

Smith, R. E., Peacock, J. A., Jenkins, A., et al. 2003, MNRAS, 341, 1311

Taruya, A., \& Hiramatsu, T. 2007 [arXiv: astro-ph/0708 . 1367]

Taylor, A. N., \& Hamilton, A. J. S. 1996, MNRAS, 282, 767

Tegmark, M., Eisenstein, D., Strauss, M., et al. 2006, Phys. Rev. D, 74, 123507

Valageas, P. 1999, A\&A, 347, 757

Valageas, P. 2002, A\&A, 382, 477

Valageas, P. 2004, A\&A, 421, 23

Valageas, P. 2007a, A\&A, 465, 725

Valageas, P. 2007b, A\&A, 476, 31

Vishniac, E. T. 1983, MNRAS, 203, 345

Zeldovich, Y. B. 1970, A\&A, 5, 84

Zinn-Justin, J. 1989, Quantum field theory and critical phenomena (Oxford: Clarendon Press) 